\title{
Diborylalkyllithium salts trigger regioselective ring opening of vinyl aziridines
}

Oriol Salvadó, Riccardo Gava*[a] and Elena Fernández*[a]

[a] Mr Oriol Salvadó Dr. Riccardo Gava, Prof. Elena Fernández

Department Química Física i Inorgànica

University Rovira i Virgili

Tarragona, Spain

E-mail: mariaelena.fernandez@urv.cat

\section{Contents:}

- General Information

- General procedure for the preparation of vinyl aziridines

- General procedures for the preparation of geminal-diborylalkanes METHOD A

- General procedures for the preparation of geminal-diborylalkanes METHOD B

- General procedure for the preparation of bis(4,4,5,5-tetramethyl-1,3,2-dioxaborolan-2-

yl)methyl)trimethylsilane

- General Procedure for the diborylalkylation ring opening of vinyl aziridines with diborylalkyllithium salts

-General Procedure for the diborylalkylation ring opening of 2-methyl-2-vinyloxirane (31) and 2-vinyloxirane (32) with diborylalkyllithium salts

- Spectral data for vinyl aziridines

- Spectral data for geminal-diborylalkanes

- Spectral data for organoboronate products

$-{ }^{1} \mathrm{H},{ }^{13} \mathrm{C}$, Spectra of vinyl aziridines

$-{ }^{1} \mathrm{H},{ }^{13} \mathrm{C},{ }^{11} \mathrm{~B}$ Spectra of geminal-diborylalkanes

$-{ }^{1} \mathrm{H},{ }^{13} \mathrm{C},{ }^{11} \mathrm{~B}$ Spectra of organoboronate products

- NOE Experiment for compound 21

- References 


\section{General Information}

Solvents and reagents: Solvents and reagents were obtained from commercial suppliers and dried and/or purified (if needed) by standard procedures. Tetrahydrofuran was dried by distillation from sodium benzophenone ketyl. (Trimethylsilyl)diazomethane solution 2.0 M in hexanes, lithium 2,2,6,6-tetramethylpiperidide as well as dienes employed were purchased from Sigma-Aldrich Inc. Bis(pinacolato)diboron was purchased from Ally Chem and used without further purification. All reactions were conducted in oven and flame-dried glassware under an inert atmosphere of argon, using Schlenk-type techniques. Flash chromatography was performed on standard silica gel (Merck Kieselgel 60 F254 400-630 mesh). Thin layer chromatography was performed on Merck Kieselgel 60 F254 which was developed using standard visualizing agents: UV fluorescence ( 254 and $366 \mathrm{~nm}$ ) or potassium permanganate/ $\Delta$. NMR spectra were recorded at a Varian Goku 400 or a Varian Mercury 400 spectrometer. ${ }^{1} \mathrm{H}$ NMR and ${ }^{13} \mathrm{C}\left\{{ }^{1} \mathrm{H}\right\}$ NMR chemical shifts $(\delta)$ are reported in ppm with the solvent resonance as the internal standard $\left(\mathrm{CHCl}_{3}: 7.26 \mathrm{ppm}(1 \mathrm{H})\right)$ and $\left(\mathrm{CDCl}_{3}: 77.16 \mathrm{ppm}\left({ }^{13} \mathrm{C}\right) \cdot{ }^{11} \mathrm{~B}\left\{{ }^{1} \mathrm{H}\right\} \mathrm{NMR}\right.$ chemical shifts $(\delta)$ are reported in ppm relative to $\left(\mathrm{CH}_{3}\right)_{2} \mathrm{O} \cdots \mathrm{BF}_{3}$. Data are reported as follows: chemical shift, multiplicity $(\mathrm{s}=$ singlet, $\mathrm{d}=$ doublet, $\mathrm{t}=$ triplet, $\mathrm{q}=$ quartet, hept $=$ heptuplet, $\mathrm{br}=$ broad, $m=$ multiplet), coupling constants $(\mathrm{Hz})$ and integration. High resolution mass spectra (HRMS) were recorded using a 6210 Time of Flight (TOF) mass spectrometer from Agilent Technologies (Waldbronn, Germany) with an ESI interface and it was performed at the Servei de Recursos Científics i Tècnics (Universitat Rovira i Virgili, Tarragona) or using a BIOTOF II Time of Flight (TOF) mass spectrometer from Bruker with an APCl interface or El interface and it was performed at the Unidade de Espectrometría de Masas e Proteómica (Universidade de Santiago de Compostela, Santiago de Compostela). GC-MS analyses were performed on a HP6890 gas chromatograph and an Agilent Technologies 5973 Mass selective detector (Waldbronn, Germany) equipped with an achiral capillary column HP-5 (30m, $0.25 \mathrm{~mm}$ i. d., $0.25 \mu \mathrm{m}$ thickness) using He as the carrier gas. 


\section{General procedure for the preparation of vinyl aziridines ${ }^{1}$}

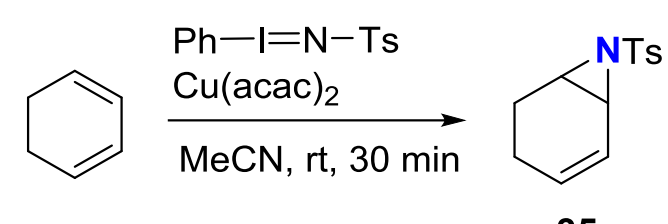

$35 a$

Phl=NTs $(0.6 \mathrm{mmol})$ was added to a solution of 1,3 -cyclohexadiene $(0.6 \mathrm{mmol})$ and copper catalyst $\mathrm{Cu}(\mathrm{acac})_{2}(0.06 \mathrm{mmol})$ in acetonitrile $(1 \mathrm{~mL})$ under argon, and the reaction followed by TLC. When the solution turned homogenous it was poured into $1 \mathrm{M} \mathrm{NaOH}(30 \mathrm{~mL})$ and extracted with diethyl ether $(1 \times 30 \mathrm{~mL})$. The organic layer was dried $\left(\mathrm{MgSO}_{4}\right)$ and the solvent removed under reduced pressure to leave either an oil or solid containing the aziridine, iodobenzene and diene starting material. This impure mixture could be dissolved in acetonitrile $(30 \mathrm{~mL})$ and washed with petroleum ether $(5 \times 10 \mathrm{~mL})$. Removal of the acetonitrile under reduced pressure produced 7-(phenylsulfonyl)-7-azabicyclo[4.1.0]hept-2-ene (35a) (104.72 mg, 70\%).

\section{General procedure for the preparation of geminal-diborylalkanes METHOD A ${ }^{2}$}

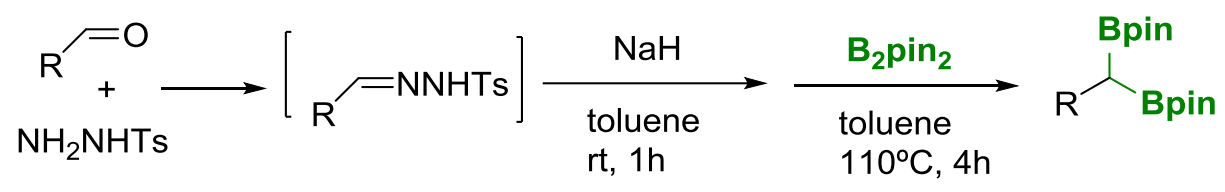

Tosylhydrazine $(14.4 \mathrm{mmol})$ were dissolved in methanol $(30 \mathrm{~mL})$ in a Schlenk flask. Then a solution of the corresponding aldehyde $(12 \mathrm{mmol})$ in $\mathrm{MeOH}$ was added to the solution of tosylhydrazine. The mixed solution was stirred for $3 \mathrm{~h}$. TLC analysis was performed until the spot of aldehyde disappeared. At this point methanol was removed and the obtained $\mathrm{N}$ tosylhydrazones was next reacted with $60 \% \mathrm{NaH}$ in $80 \mathrm{~mL}$ of dry toluene. The mixture was stirred at room temperature for $1 \mathrm{~h}$. Then $\mathrm{B}_{2} \mathrm{pin}_{2}(14.4 \mathrm{mmol})$ was added along with additional $5 \mathrm{~mL}$ of

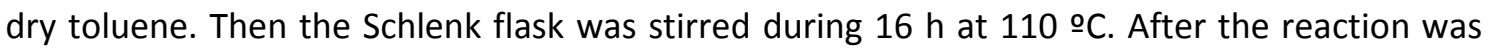
cooled down to room temperature, the obtained suspension was filtered with Celite ${ }^{\circledR}$ and solvent was concentrated on a rotary evaporator. The crude residue was purified by silica gel flash chromatography to afford the geminal-diborylalkanes. 


\section{General procedure for the preparation of geminal-diborylalkanes METHOD B ${ }^{3}$}

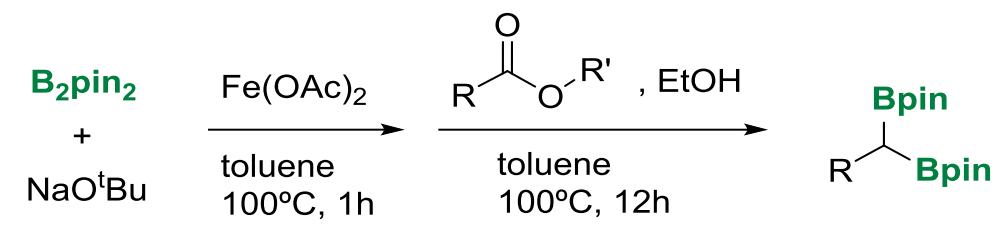

In the glove box, in an oven-dried Schlenk tube equipped with a magnetic bar, $\mathrm{B}_{2} \mathrm{pin}_{2}$ (4 mmol, 4 equiv.), $\mathrm{NaO}^{\mathrm{t}} \mathrm{Bu}$ ( $3 \mathrm{mmol}, 3$ equiv.), $\mathrm{Fe}(\mathrm{OAc})_{2}(0.1 \mathrm{mmol}, 0.1$ equiv.) and dry toluene $(7 \mathrm{~mL})$ were added. The Schlenk flask was sealed and taken out of the glove box and the mixture was stirred at $100{ }^{\circ} \mathrm{C}$ for $1 \mathrm{~h}$. Then, the corresponding ester ( $1 \mathrm{mmol}, 1$ equiv.) and dry EtOH ( $1 \mathrm{mmol}$, 1 equiv.) were added sequentially and the resulting mixture was stirred at $100 \circ \mathrm{C}$ for $12 \mathrm{~h}$. The reaction was quenched with ethyl acetate and water. The layers were separated and the aqueous layer was extracted with ethyl acetate $(3 \times 45 \mathrm{~mL})$. The organic layer was dried over anhydrous $\mathrm{MgSO}_{4}$, filtered and the solvent was removed by using rotatory evaporator. The crude was purified by silica gel flash chromatography to afford the geminal-diborylalkanes.

\section{General procedure for the preparation of bis $(4,4,5,5$-tetramethyl-1,3,2-dioxaborolan-2-} yl)methyl)trimethylsilane ${ }^{4}$

$$
\mathrm{Me}_{3} \mathrm{Si} /=\mathrm{N}_{2} \frac{\mathrm{B}_{2} \mathrm{pin}_{2}}{\begin{array}{l}
\text { n-hexane } \\
110^{\circ} \mathrm{C}, 24 \mathrm{~h}
\end{array}} \mathrm{Me}_{3} \mathrm{Si} \overbrace{\text { Bpin }}^{\text {Bpin }}
$$

In the glove-box, an oven-dried Teflon screw-cap Schlenk reaction flask equipped with a magnetic stir bar was charged with 4 mmol (1 equiv.) of bis(pinacolato)diboron. Then, 2 equiv. $(8 \mathrm{mmol})$ of a $2.0 \mathrm{M}$ solution in hexane of (trimethylsilyl)diazomethane were added dropwise. After stirring the mixture in the glove-box for $5 \mathrm{~min}$ the Schlenk flask was sealed and heated at $110{ }^{\circ} \mathrm{C}$ for $24 \mathrm{~h}$ while constantly stirring. The reaction was cooled at room temperature, the solvent was gently concentrated on a rotary evaporator and the resulting crude purified by silica gel flash chromatography to afford the bis(4,4,5,5-tetramethyl-1,3,2-dioxaborolan-2yl)methyl)trimethylsilane. 
General Procedure for the diborylalkylation ring opening of vinyl aziridines with diborylalkyllithium salts

A Schlenk-tube equipped with a magnetic stir bar was charged with geminal-diborylalkanes ( 0.5 mmol, 1 equiv) and LiTMP ( $0.6 \mathrm{mmol}, 1.2$ equiv) in dry THF as solvent $(2 \mathrm{~mL})$. The mixture was stirred during $30 \mathrm{~min}$ at $0 \stackrel{\circ}{ } \mathrm{C}$. Then, a solution of the vinyl aziridine $(0.4 \mathrm{mmol}, 0.8$ equiv) in $1 \mathrm{~mL}$ of THF was added. The reaction was stirred during $10 \mathrm{~min}$ at $0 \stackrel{\circ}{ }$, followed by $16 \mathrm{~h}$ at room temperature. The crude residue was purified by silica gel flash chromatography to afford the desired product.

Example Procedure for the diborylalkylation ring opening of 1-tosyl-2-vinylaziridine (1) with diborylmethyllithium salt

A Schlenk-tube equipped with a magnetic stir bar was charged with 2,2'-(ethane-1,1diyl)bis(4,4,5,5-tetramethyl-1,3,2-dioxaborolane) (2) (1 mmol, $281.99 \mathrm{mg}, 1$ equiv) and LiTMP (1.2 mmol, $176.63 \mathrm{mg}, 1.2$ equiv) in dry THF as solvent $(2 \mathrm{~mL})$. The mixture was stirred during 30 $\min$ at $0 \stackrel{\circ}{ }{ }^{\circ}$. Then, a solution of 1-tosyl-2-vinylaziridine (1) $(0.8 \mathrm{mmol}, 178.63 \mathrm{mg}, 0.8$ equiv) in 1 $\mathrm{mL}$ of THF was added. The reaction was stirred during $10 \mathrm{~min}$ at $0 \stackrel{\circ}{ } \mathrm{C}$, followed by $16 \mathrm{~h}$ at room temperature. The reaction mixture was concentrated and the crude residue was purified by silica gel flash chromatography to afford the desired products (E)-N-(5,5-bis(4,4,5,5-tetramethyl1,3,2-dioxaborolan-2-yl)hex-2-en-1-yl)-4-methylbenzenesulfonamide (3) (35\%, $141 \mathrm{mg}$ ) and N(5,5-bis(4,4,5,5-tetramethyl-1,3,2-dioxaborolan-2-yl)hex-1-en-3-yl)-4-

methylbenzenesulfonamide (4) $(64 \%, 259 \mathrm{mg})$

\section{General Procedure for the diborylalkylation ring opening of 2-methyl-2-vinyloxirane (31) and} 2-vinyloxirane (32) with diborylalkyllithium salts

A Schlenk-tube equipped with a magnetic stir bar was charged with geminal-diborylalkanes $(0.5$ mmol, 1 equiv) and LiTMP ( $0.6 \mathrm{mmol}, 1.2$ equiv) in dry THF as solvent $(2 \mathrm{~mL})$. The mixture was stirred during $30 \mathrm{~min}$ at $0 \stackrel{\mathrm{O}}{\mathrm{C}}$. Then, a solution of the vinyl epoxide $(0.4 \mathrm{mmol}, 0.8$ equiv) in $1 \mathrm{~mL}$ of THF was added. The reaction was stirred during $10 \mathrm{~min}$ at $0 \stackrel{\circ}{ } \mathrm{C}$, followed by $16 \mathrm{~h}$ at room temperature. The crude residue was purified by silica gel flash chromatography to afford the desired product. 


\section{Spectral data for vinyl aziridines}

\section{1-tosyl-2-vinylaziridine (1)}<smiles>C=CC1CC1</smiles>

Purified by flash column chromatography (hexane:ethyl acetate $=20: 1)$ yielded $\mathbf{1}(81 \%, 108$ $\mathrm{mg}$ ) as a white solid.

${ }^{1} \mathrm{H}$ NMR $\left(\mathrm{CDCl}_{3}, 400 \mathrm{MHz}\right) \delta 7.89-7.63(\mathrm{~m}, 2 \mathrm{H}), 7.26(\mathrm{~d}, \mathrm{~J}=7.3 \mathrm{~Hz}, 2 \mathrm{H}), 5.51-5.38(\mathrm{~m}, 1 \mathrm{H}), 5.35$ $(\mathrm{dt}, \mathrm{J}=17.2,1.7 \mathrm{~Hz}, 1 \mathrm{H}), 5.16(\mathrm{dt}, \mathrm{J}=9.8,1.7 \mathrm{~Hz}, 1 \mathrm{H}), 3.28-3.11(\mathrm{~m}, 1 \mathrm{H}), 2.74-2.64(\mathrm{~m}, 1 \mathrm{H})$, $2.37(\mathrm{~s}, 3 \mathrm{H}), 2.14(\mathrm{dd}, \mathrm{J}=4.6,1.3 \mathrm{~Hz}, 1 \mathrm{H})$.

${ }^{13} \mathrm{C} \mathrm{NMR}\left(\mathrm{CDCl}_{3}, 100 \mathrm{MHz}\right) \delta$ 144.59, 135.15, 132.97, 129.73, 127.84, 120.32, 40.98, 34.18, 21.64. HRMS (ESI) for $\mathrm{C}_{11} \mathrm{H}_{13} \mathrm{NNaO}_{2} \mathrm{~S}^{+}\left[\mathrm{M}+\mathrm{Na}^{+}\right]^{+}$: calculated: 246.0564 , found: 246.0563 .

\section{(E)-2-styryl-1-tosylaziridine (14)}<smiles>[AsH2+]=CC1CC1</smiles>

Purified by flash column chromatography (hexane:ethyl acetate $=20: 1)$ yielded 14 (63\%, 113 $\mathrm{mg}$ ) as a white solid.

${ }^{1} \mathrm{H}$ NMR $\left(\mathrm{CDCl}_{3}, 400 \mathrm{MHz}\right) \delta 7.77(\mathrm{~d}, \mathrm{~J}=8.3 \mathrm{~Hz}, 2 \mathrm{H}), 7.34-7.07(\mathrm{~m}, 7 \mathrm{H}), 6.65(\mathrm{~d}, \mathrm{~J}=15.9 \mathrm{~Hz}, 1 \mathrm{H})$, $5.76(\mathrm{dd}, \mathrm{J}=15.9,7.9 \mathrm{~Hz}, 1 \mathrm{H}), 3.45-3.29(\mathrm{~m}, 1 \mathrm{H}), 2.79(\mathrm{~d}, \mathrm{~J}=7.1 \mathrm{~Hz}, 1 \mathrm{H}), 2.36(\mathrm{~s}, 3 \mathrm{H}), 2.24(\mathrm{~d}, \mathrm{~J}$ $=4.5 \mathrm{~Hz}, 1 \mathrm{H})$.

${ }^{13} \mathrm{C}$ NMR $\left(\mathrm{CDCl}_{3}, 100 \mathrm{MHz}\right) \delta 144.65,135.79,135.17,135.12,129.79,128.65,128.27,127.87$, $126.49,124.05,41.22,34.64,21.66$.

HRMS (ESI) for $\mathrm{C}_{17} \mathrm{H}_{18} \mathrm{NO}_{2} \mathrm{~S}^{+}\left[\mathrm{M}+\mathrm{H}^{+}\right]^{+}$: calculated: 300.1058 , found: 300.1054

(E)-2-(1-phenylprop-1-en-1-yl)-1-tosylaziridine (17)<smiles>C/C=C(\c1ccccc1)C1CC1</smiles>

Purified by flash column chromatography (hexane:ethyl acetate $=20: 1$ ) yielded 17 (65\%, 122 $\mathrm{mg}$ ) as a white solid. 
${ }^{1} \mathrm{H}$ NMR $\left(\mathrm{CDCl}_{3}, 400 \mathrm{MHz}\right) \delta 7.71(\mathrm{~d}, \mathrm{~J}=8.3 \mathrm{~Hz}, 2 \mathrm{H}), 7.29-7.10(\mathrm{~m}, 5 \mathrm{H}), 7.03-6.90(\mathrm{~m}, 2 \mathrm{H}), 5.81$ $(q, J=6.9,1 H), 3.44-3.32(m, 1 H), 2.63(d, J=7.0 \mathrm{~Hz}, 1 \mathrm{H}), 2.36(\mathrm{~s}, 3 \mathrm{H}), 2.07(\mathrm{~d}, \mathrm{~J}=4.6 \mathrm{~Hz}, 1 \mathrm{H})$, $1.47(\mathrm{~d}, \mathrm{~J}=6.9,3 \mathrm{H})$.

${ }^{13} \mathrm{C}$ NMR $\left(\mathrm{CDCl}_{3}, 100 \mathrm{MHz}\right) \delta 144.36,136.37,135.33,135.18,129.63,129.00,128.10,127.90$, $127.55,127.20,43.89,33.09,21.64,14.61$.

HRMS (ESI) for $\mathrm{C}_{18} \mathrm{H}_{19} \mathrm{NNaO}_{2}{ }^{+}\left[\mathrm{M}+\mathrm{Na}^{+}\right]^{+}$: calculated: 336.1034 , found: 336.1033

2-methyl-1-tosyl-2-vinylaziridine (20) with traces of 2-(prop-1-en-2-yl)-1-tosylaziridine<smiles>C=CC1(C)CC1</smiles>

Purified by flash column chromatography (hexane:ethyl acetate $=20: 1$ ) provided a mixture of 20 with traces of 2-(prop-1-en-2-yl)-1-tosylaziridine in 79\%, $112 \mathrm{mg}$.

${ }^{1} \mathrm{H}$ NMR $\left(\mathrm{CDCl}_{3}, 400 \mathrm{MHz}\right) \delta 7.75\left(\mathrm{~m}, 2 \mathrm{H}_{1}, 2 \mathrm{H}_{1 \mathrm{a}}\right), 7.28-7.16\left(\mathrm{~m}, 2 \mathrm{H}_{1}, 2 \mathrm{H}_{1 \mathrm{a}}\right), 5.89$ (dd, J = 17.2, 10.7 $\left.\mathrm{Hz}, 1 \mathrm{H}_{1}\right), 5.33\left(\mathrm{dd}, \mathrm{J}=17.3,0.8 \mathrm{~Hz}, 1 \mathrm{H}_{1}\right), 5.24\left(\mathrm{dd}, \mathrm{J}=10.6,0.8 \mathrm{~Hz}, 1 \mathrm{H}_{1}\right), 4.98\left(\mathrm{~m}, 1 \mathrm{H}_{1 \mathrm{a}}\right), 4.87(\mathrm{~m}$, $\left.1 \mathrm{H}_{1 \mathrm{a}}\right), 3.18\left(\mathrm{dd}, \mathrm{J}=7.1,4.6 \mathrm{~Hz}, 1 \mathrm{H}_{1 \mathrm{a}}\right), 2.64\left(\mathrm{~d}, \mathrm{~J}=7.1 \mathrm{~Hz}, 1 \mathrm{H}_{1 \mathrm{a}}\right), 2.55\left(\mathrm{~d}, \mathrm{~J}=5.9 \mathrm{~Hz}, 2 \mathrm{H}_{1}\right), 2.36(\mathrm{~s}$, $\left.3 \mathrm{H}_{1 \mathrm{a}}\right), 2.35\left(\mathrm{~s}, 3 \mathrm{H}_{1}\right), 2.22\left(\mathrm{~d}, \mathrm{~J}=4.6 \mathrm{~Hz}, 1 \mathrm{H}_{1 \mathrm{a}}\right), 1.57\left(\mathrm{~s}, 3 \mathrm{H}_{1}\right), 1.50\left(\mathrm{~m}, 3 \mathrm{H}_{1 \mathrm{a}}\right)$.

${ }^{13} \mathrm{C} \mathrm{NMR}\left(\mathrm{CDCl}_{3}, 100 \mathrm{MHz}\right) \delta$ 144.54(1a), 143.95(1), 138.83(1a), 137.64(1), 137.01(1), 135.14(1a), 129.70(1a), 129.52(1), 127.88(1a), 127.40(1), 118.29(1), 115.60(1a), 49.83(1), 43.35(1a), 42.03(1), 32.48(1a), 21.64(1a), 21.59(1), 18.57(1), 17.79(1a).

HRMS (ESI) for ${ }_{12} \mathrm{H}_{16} \mathrm{NO}_{2} \mathrm{~S}^{+}\left[\mathrm{M}+\mathrm{H}^{+}\right]^{+}$: calculated: 238.0898, found: 238.0901

\section{7-tosyl-7-azabicyclo[4.1.0]hept-2-ene (35)}<smiles>CC1(C)C2C=CCCC21S</smiles>

35

Purified by flash column chromatography (hexane:ethyl acetate $=20: 1)$ yielded $\mathbf{3 5}$ (70\%, 105 $\mathrm{mg})$ as a white solid.

${ }^{1} \mathrm{H}$ NMR $\left(\mathrm{CDCl}_{3}, 400 \mathrm{MHz}\right) \delta 7.74(\mathrm{~d}, \mathrm{~J}=6.7 \mathrm{~Hz}, 1 \mathrm{H}), 7.25(\mathrm{~d}, \mathrm{~J}=7.9 \mathrm{~Hz}, 2 \mathrm{H}), 5.81(\mathrm{~m}, 2 \mathrm{H}), 3.29-$ $3.18(m, 1 H), 3.16-3.04(m, 1 H), 2.36(s, 3 H), 1.96(m, 3 H), 1.49-1.41(m, 1 H)$.

${ }^{13} \mathrm{C} \mathrm{NMR}\left(\mathrm{CDCl}_{3}, 100 \mathrm{MHz}\right) \delta 144.19,135.60,133.53,129.65,127.67,120.41,41.65,36.58$, $21.62,20.43,18.61$.

HRMS (ESI) for $\mathrm{C}_{13} \mathrm{H}_{15} \mathrm{NNaO}_{2} \mathrm{~S}^{+}\left[\mathrm{M}+\mathrm{Na}^{+}\right]^{+}$: calculated: 272.0721 , found: 272.0721. 
Spectral data for geminal-diborylalkanes

2,2'-(ethane-1,1-diyl)bis(4,4,5,5-tetramethyl-1,3,2-dioxaborolane) (2)<smiles>CC(B1OC(C)(C)C(C)(C)O1)B1OC(C)(C)C(C)(C)O1</smiles>

Synthetized using General Procedure B. Purified by flash column chromatography (hexane:ethyl acetate $=20: 1)$ yielded $\mathbf{2}(55 \%, 155 \mathrm{mg})$ as a colourless oil.

${ }^{1} \mathrm{H}_{\mathrm{NMR}}\left(\mathrm{CDCl}_{3}, 400 \mathrm{MHz}\right) \delta 1.22-1.09(\mathrm{~m}, 24 \mathrm{H}), 1.01-0.92(\mathrm{~m}, 3 \mathrm{H}), 0.65(\mathrm{q}, \mathrm{J}=5.2,3.1 \mathrm{~Hz}$, $1 \mathrm{H})$.

${ }^{13} \mathrm{C} \mathrm{NMR}\left(\mathrm{CDCl}_{3}, 100 \mathrm{MHz}\right) \delta 82.88,24.82,24.53,9.04$.

${ }^{11}$ B NMR $\left(\mathrm{CDCl}_{3}, 128.3 \mathrm{MHz}\right) \delta 33.96$.

HRMS (ESI) for $\mathrm{C}_{14} \mathrm{H}_{29} \mathrm{~B}_{2} \mathrm{O}_{4}{ }^{+}\left[\mathrm{M}+\mathrm{H}^{+}\right]^{+}$: calculated: 283.2252 , found: 283.2254 .

\section{2,2'-(2,2-dimethylpropane-1,1-diyl)bis(4,4,5,5-tetramethyl-1,3,2-dioxaborolane) (5)}<smiles>CC(C)(C)C(B1OC(C)(C)C(C)(C)O1)B1OC(C)(C)C(C)(C)O1</smiles>

5

Synthetized using General Procedure A. Purified by flash column chromatography (hexane:ethyl acetate $=20: 1)$ yielded $5(31 \%, 1.19 \mathrm{~g})$ as a white-yellowish solid. ${ }^{1} \mathbf{H}$ NMR $\left(\mathrm{CDCl}_{3}, 400 \mathrm{MHz}\right) \delta 1.15(\mathrm{~m}, 25 \mathrm{H}), 0.99(\mathrm{~s}, 9 \mathrm{H})$.

${ }^{13} \mathrm{C} \mathrm{NMR}\left(\mathrm{CDCl}_{3}, 100 \mathrm{MHz}\right) \delta 82.61,31.88,31.31,24.92,24.53$.

${ }^{11}$ B NMR $\left(\mathrm{CDCl}_{3}, 128.3 \mathrm{MHz}\right) \delta 33.28$.

HRMS (ESI) for $\mathrm{C}_{17} \mathrm{H}_{38} \mathrm{~B}_{2} \mathrm{NO}_{4}\left[\mathrm{M}+\mathrm{NH}_{4}^{+}\right]^{+}$: calculated: 342.2987 , found: 342.298

(bis(4,4,5,5-tetramethyl-1,3,2-dioxaborolan-2-yl)methyl)trimethylsilane (8)<smiles>CC1(C)OB(C(B2OC(C)(C)C(C)(C)O2)[Si](C)(C)C)OC1(C)C</smiles>

Purified by flash columns chromatography (hexane:ethyl acetate $=20: 1$ ) yielded $8(83 \%, 1.17 \mathrm{~g})$ as a white solid. 
${ }^{1} \mathrm{H}$ NMR $\left(\mathrm{CDCl}_{3}, 400 \mathrm{MHz}\right) \delta 1.23(\mathrm{~s}, 12 \mathrm{H}), 1.21(\mathrm{~s}, 12 \mathrm{H}), 0.30(\mathrm{~s}, 1 \mathrm{H}), 0.10(\mathrm{~s}, 9 \mathrm{H})$.

${ }^{13} \mathrm{C}$ NMR $\left(\mathrm{CDCl}_{3}, 100 \mathrm{MHz}\right) \delta 82.12,24.45,23.98,0.00$

${ }^{11}$ B NMR $\left(\mathrm{CDCl}_{3}, 128.3 \mathrm{MHz}\right) \delta 33.04$.

HRMS (ESI) for $\mathrm{C}_{16} \mathrm{H}_{34} \mathrm{~B}_{2} \mathrm{O}_{4} \mathrm{SiNa}\left[\mathrm{M}+\mathrm{Na}^{+}\right]^{+}$: calculated: 363.231 , found: 363.2309 .

2,2'-((tetrahydro-2H-pyran-4-yl)methylene)bis(4,4,5,5-tetramethyl-1,3,2-dioxaborolane) (11)<smiles>CC1(C)OB(C(B2OC(C)(C)C(C)(C)O2)C2CCOCC2)OC1(C)C</smiles>

11

Synthetized using General Procedure B. Purified by flash column chromatography (hexane:ethyl acetate $=20: 1)$ yielded $\mathbf{1 1}(35 \%, 123 \mathrm{mg})$ as a white solid.

${ }^{1} \mathrm{H}$ NMR $\left(\mathrm{CDCl}_{3}, 400 \mathrm{MHz}\right) \delta 3.82(\mathrm{dd}, \mathrm{J}=11.3,3.8 \mathrm{~Hz}, 2 \mathrm{H}), 3.33(\mathrm{td}, \mathrm{J}=11.9,2.0 \mathrm{~Hz}, 2 \mathrm{H}), 1.83(\mathrm{~m}$, 1H), $1.62(\mathrm{dd}, \mathrm{J}=12.9,4.0 \mathrm{~Hz}, 2 \mathrm{H}), 1.27(\mathrm{~m}, 2 \mathrm{H}), 1.16(\mathrm{~s}, 12 \mathrm{H}), 1.15(\mathrm{~s}, 12 \mathrm{H}), 0.62(\mathrm{~d}, \mathrm{~J}=10.2 \mathrm{~Hz}$, $1 \mathrm{H})$.

${ }^{13} \mathrm{C} \mathrm{NMR}\left(\mathrm{CDCl}_{3}, 100 \mathrm{MHz}\right) \delta 83.03,68.46,35.46,33.01,24.92,24.56$

${ }^{11}$ B NMR $\left(\mathrm{CDCl}_{3}, 128.3 \mathrm{MHz}\right) \delta 34.05$.

HRMS (ESI) for $\mathrm{C}_{18} \mathrm{H}_{35} \mathrm{~B}_{2} \mathrm{O}_{5}{ }^{+}\left[\mathrm{M}+\mathrm{H}^{+}\right]^{+}$: calculated: 353.2671 , found: 353.2676

2,2'-(3-phenylpropane-1,1-diyl)bis(4,4,5,5-tetramethyl-1,3,2-dioxaborolane) (25)<smiles>CC1(C)OB(C(CCc2ccccc2)B2OC(C)(C)C(C)(C)O2)OC1(C)C</smiles>

Synthetized using General procedure A. Purified by flash columns chromatography (hexane:ethyl acetate $=20: 1$ ) yielded $\mathbf{2 5}(68 \%, 3.01 \mathrm{~g}$ ) as an oil.

${ }^{1} \mathrm{H}$ NMR $\left(\mathrm{CDCl}_{3}, 400 \mathrm{MHz}\right) \delta 7.21-7.01(\mathrm{~m}, 5 \mathrm{H}), 2.51(\mathrm{dd}, \mathrm{J}=9.2,6.8 \mathrm{~Hz}, 2 \mathrm{H}), 1.84-1.72(\mathrm{~m}$, $2 \mathrm{H}), 1.16(\mathrm{~s}, 12 \mathrm{H}), 1.15(\mathrm{~s}, 12 \mathrm{H}), 0.74,(\mathrm{t}, \mathrm{J}=7.9 \mathrm{~Hz}, 1 \mathrm{H})$.

${ }^{13} \mathrm{C} \mathrm{NMR}\left(\mathrm{CDCl}_{3}, 100 \mathrm{MHz}\right) \delta 142.92,128.56,128.08,125.45,82.95,38.71,27.99,24.90,24.49$.

${ }^{11}$ B NMR $\left(\mathrm{CDCl}_{3}, 128.3 \mathrm{MHz}\right) \delta 34.0$.

HRMS (ESI) for $\mathrm{C}_{21} \mathrm{H}_{38} \mathrm{NB}_{2} \mathrm{O}_{4}\left[\mathrm{M}+\mathrm{NH}_{4}{ }^{+}\right]^{+}$: calculated: 390.2987 , found: 390.2989 
2,2'-(2-phenylethane-1,1-diyl)bis(4,4,5,5-tetramethyl-1,3,2-dioxaborolane) (27)<smiles>[Z7]CC(B1OC(C)(C)C(C)(C)O1)B1OC(C)(C)C(C)(C)O1</smiles>

Synthetized using General procedure A. Purified by flash columns chromatography (hexane:ethyl acetate $=20: 1)$ yielded $\mathbf{2 7}(47 \%, 2.05 \mathrm{~g})$ as a yellowish oil.

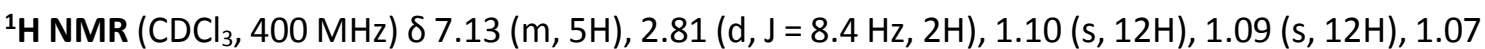
$(\mathrm{m}, 1 \mathrm{H})$.

${ }^{13} \mathrm{C} \mathrm{NMR}\left(\mathrm{CDCl}_{3}, 100 \mathrm{MHz}\right) \delta 144.47,128.34,127.95,125.34,83.09,31.30,25.03,24.78,24.52$.

${ }^{11}$ B NMR $\left(\mathrm{CDCl}_{3}, 128.3 \mathrm{MHz}\right) \delta 33.77$.

HRMS (ESI) for $\mathrm{C}_{20} \mathrm{H}_{36} \mathrm{~B}_{2} \mathrm{NO}_{4}\left[\mathrm{M}+\mathrm{NH}_{4}{ }^{+}\right]^{+}$: calculated: 376.2830 , found: 376.2832

2,2'-(p-tolylmethylene)bis(4,4,5,5-tetramethyl-1,3,2-dioxaborolane) (29)<smiles></smiles>

Synthetized using General procedure A. Purified by flash columns chromatography (hexane:ethyl acetate $=20: 1)$ yielded $\mathbf{2 9}(47 \%, 2.1 \mathrm{~g})$ as a white solid.

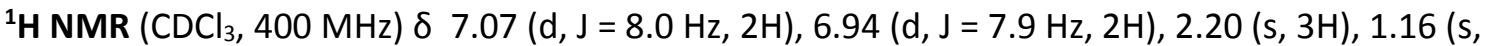
$1 \mathrm{H}), 1.15(\mathrm{~s}, 12 \mathrm{H}), 1.12(\mathrm{~s}, 12 \mathrm{H})$.

${ }^{13} \mathrm{C} \mathrm{NMR}\left(\mathrm{CDCl}_{3}, 100 \mathrm{MHz}\right) \delta 136.22,128.97,128.71,83.30,30.94,24.69,24.62,20.98$.

${ }^{11} \mathbf{B}$ NMR $\left(\mathrm{CDCl}_{3}, 128.3 \mathrm{MHz}\right) \delta 33.26$.

HRMS (ESI) for $\mathrm{C}_{20} \mathrm{H}_{36} \mathrm{~B}_{2} \mathrm{NO}_{4}\left[\mathrm{M}+\mathrm{NH}_{4}{ }^{+}\right]^{+}$: calculated: 376.283 , found: 376.283 


\section{Spectral data for organoboronate products}

(E)-N-(5,5-bis(4,4,5,5-tetramethyl-1,3,2-dioxaborolan-2-yl)hex-2-en-1-yl)-4methylbenzenesulfonamide (3)

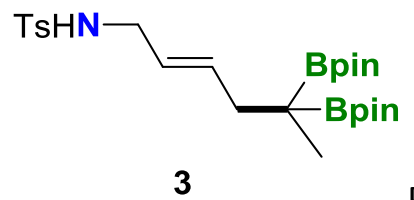

Purified by flash column chromatography (hexane:ethyl acetate $=9: 1$ )

yielded 3 (35\%, $71 \mathrm{mg})$ as a white solid.

${ }^{1} \mathrm{H} \mathrm{NMR}\left(\mathrm{CDCl}_{3}, 400 \mathrm{MHz}\right) \delta 7.66(\mathrm{~d}, \mathrm{~J}=8.3 \mathrm{~Hz}, 2 \mathrm{H}), 7.23(\mathrm{~d}, \mathrm{~J}=7.9 \mathrm{~Hz}, 2 \mathrm{H}), 5.56-5.41(\mathrm{~m}, 1 \mathrm{H})$, $5.30-5.16(\mathrm{~m}, 1 \mathrm{H}), 4.25(\mathrm{t}, \mathrm{J}=6.0 \mathrm{~Hz}, 1 \mathrm{H}), 3.43(\mathrm{t}, \mathrm{J}=6.2 \mathrm{~Hz}, 2 \mathrm{H}), 2.35(\mathrm{~s}, 3 \mathrm{H}), 2.10(\mathrm{~d}, \mathrm{~J}=7.2$ $\mathrm{Hz}, 2 \mathrm{H}), 1.12(\mathrm{~m}, 24 \mathrm{H}), 0.87(\mathrm{~s}, 3 \mathrm{H})$.

${ }^{13} \mathrm{C} \mathrm{NMR}\left(\mathrm{CDCl}_{3}, 100 \mathrm{MHz} \delta 143.32,137.11,134.54,129.69,127.14,125.40,83.09,45.43,36.41\right.$, $24.71,24.60,21.51,15.85$.

${ }^{11} \mathrm{~B}$ NMR $\left(\mathrm{CDCl}_{3}, 128.3 \mathrm{MHz}\right) \delta 34.16$.

HRMS (ESI) for $\mathrm{C}_{25} \mathrm{H}_{42} \mathrm{~B}_{2} \mathrm{NO}_{6} \mathrm{~S}$ [M+H'] ${ }^{+}$: calculated: 506.2919 , found: 506.2917 .

$\mathrm{N}$-(5,5-bis(4,4,5,5-tetramethyl-1,3,2-dioxaborolan-2-yl)hex-1-en-3-yl)-4methylbenzenesulfonamide (4)

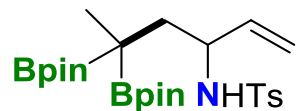

4

Purified by flash column chromatography (hexane:ethyl acetate $=9: 1)$ yielded 4 (64\%, $129 \mathrm{mg}$ ) as a white solid.

${ }^{1} \mathrm{H}$ NMR $\left(\mathrm{CDCl}_{3}, 400 \mathrm{MHz}\right) \delta 7.61(\mathrm{~d}, \mathrm{~J}=8.3 \mathrm{~Hz}, 2 \mathrm{H}), 7.16(\mathrm{~d}, \mathrm{~J}=7.8 \mathrm{~Hz}, 2 \mathrm{H}), 5.35$ (ddd, J = 16.8, 10.0, 7.7 Hz, 2H), $4.87(\mathrm{dt}, \mathrm{J}=17.1,1.1 \mathrm{~Hz}, 1 \mathrm{H}), 4.71(\mathrm{dt}, \mathrm{J}=10.2,1.0 \mathrm{~Hz}, 1 \mathrm{H}), 3.87-3.73(\mathrm{~m}, 1 \mathrm{H})$, $2.32(\mathrm{~s}, 3 \mathrm{H}), 1.80(\mathrm{dd}, \mathrm{J}=14.5,10.4 \mathrm{~Hz}, 1 \mathrm{H}), 1.46(\mathrm{~m}, 1 \mathrm{H}), 1.22(\mathrm{~s}, 6 \mathrm{H}), 1.20(\mathrm{~s}, 6 \mathrm{H}), 1.12(\mathrm{~s}, 12 \mathrm{H})$, $0.97(s, 3 H)$.

${ }^{13} \mathrm{C} \mathrm{NMR}\left(\mathrm{CDCl}_{3}, 100 \mathrm{MHz}\right) \delta 142.72,139.25,138.74,129.17,127.49,115.51,83.74,83.29,54.91$, $38.64,25.23,24.68,24.61,24.56,21.48,14.89$.

${ }^{11}$ B NMR $\left(\mathrm{CDCl}_{3}, 128.3 \mathrm{MHz}\right) \delta 34.29$.

HRMS (ESI) for $\mathrm{C}_{25} \mathrm{H}_{42} \mathrm{~B}_{2} \mathrm{NO}_{6} \mathrm{~S}\left[\mathrm{M}+\mathrm{H}^{+}\right]^{+}$: calculated: 506.2919, found: 506.2913 . 
(E)-N-(6,6-dimethyl-5,5-bis(4,4,5,5-tetramethyl-1,3,2-dioxaborolan-2-yl)hept-2-en-1-yl)-4methylbenzenesulfonamide (6)

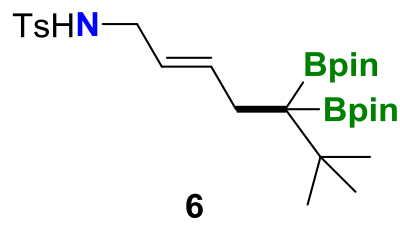

Purified by flash column chromatography (hexane:ethyl acetate $=9: 1)$ yielded $6(38 \%, 83 \mathrm{mg}$ ) as a white solid.

${ }^{1} \mathrm{H}$ NMR $\left(\mathrm{CDCl}_{3}, 400 \mathrm{MHz}\right) \delta 7.67(\mathrm{~d}, \mathrm{~J}=8.3 \mathrm{~Hz}, 2 \mathrm{H}), 7.23(\mathrm{~d}, \mathrm{~J}=8.0 \mathrm{~Hz}, 2 \mathrm{H}), 5.79-5.62(\mathrm{~m}, 1 \mathrm{H})$, $5.33-5.12(\mathrm{~m}, 1 \mathrm{H}), 4.22(\mathrm{t}, \mathrm{J}=5.9 \mathrm{~Hz}, 1 \mathrm{H}), 3.49-3.32(\mathrm{~m}, 2 \mathrm{H}), 2.35(\mathrm{~s}, 3 \mathrm{H}), 2.20(\mathrm{dd}, \mathrm{J}=7.0,1.5$ $\mathrm{Hz}, 2 \mathrm{H}), 1.15(\mathrm{~s}, 24 \mathrm{H}), 0.92(\mathrm{~s}, 9 \mathrm{H})$.

${ }^{13} \mathrm{C} \mathrm{NMR}\left(\mathrm{CDCl}_{3}, 100 \mathrm{MHz}\right) \delta 143.25,138.01,137.17,129.65,127.16,123.44,82.57,45.59,34.00$, $32.87,29.60,24.90,24.77,21.50$.

${ }^{11}$ B NMR $\left(\mathrm{CDCl}_{3}, 128.3 \mathrm{MHz}\right) \delta 33.28$.

HRMS (ESI) for $\mathrm{C}_{28} \mathrm{H}_{48} \mathrm{~B}_{2} \mathrm{NO}_{6} \mathrm{~S}$ [M+H+ $]^{+}$: calculated: 548.3388 , found: 548.3391 .

N-(6,6-dimethyl-5,5-bis(4,4,5,5-tetramethyl-1,3,2-dioxaborolan-2-yl)hept-1-en-3-yl)-4methylbenzenesulfonamide (7)

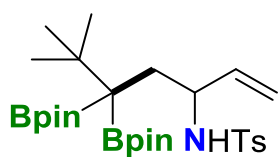

7

Purified by flash column chromatography (hexane:ethyl acetate $=9: 1)$ yielded 7 (53\%, $116 \mathrm{mg}$ ) as a white solid.

${ }^{1} \mathrm{H}$ NMR $\left(\mathrm{CDCl}_{3}, 400 \mathrm{MHz}\right) \delta 7.58(\mathrm{~d}, \mathrm{~J}=8.3 \mathrm{~Hz}, 2 \mathrm{H}), 7.13(\mathrm{~d}, \mathrm{~J}=8.0 \mathrm{~Hz}, 2 \mathrm{H}), 6.54(\mathrm{~d}, \mathrm{~J}=4.8 \mathrm{~Hz}$, $1 \mathrm{H}), 5.24(\mathrm{ddd}, \mathrm{J}=17.0,10.0,8.3 \mathrm{~Hz}, 1 \mathrm{H}), 4.92(\mathrm{dd}, \mathrm{J}=17.0,1.6 \mathrm{~Hz}, 1 \mathrm{H}), 4.68(\mathrm{dd}, \mathrm{J}=10.1,1.6$ $\mathrm{Hz}, 1 \mathrm{H}), 4.13-4.02(\mathrm{~m}, 1 \mathrm{H}), 2.31(\mathrm{~s}, 3 \mathrm{H}), 1.65(\mathrm{~d}, \mathrm{~J}=4.2 \mathrm{~Hz}, 2 \mathrm{H}), 1.20(\mathrm{~s}, 18 \mathrm{H}), 1.18(\mathrm{~s}, 6 \mathrm{H}), 0.97$ $(s, 9 H)$.

${ }^{13} \mathrm{C} \mathrm{NMR}\left(\mathrm{CDCl}_{3}, 100 \mathrm{MHz}\right) \delta 142.19,140.09,139.91,128.96,127.36,115.20,83.36,83.25,57.07$, $35.77,34.48,29.54,25.23,25.20,25.04,24.37,21.44$.

${ }^{11} \mathbf{B}$ NMR $\left(\mathrm{CDCl}_{3}, 128.3 \mathrm{MHz}\right) \delta 33.48$.

HRMS (ESI) for $\mathrm{C}_{28} \mathrm{H}_{48} \mathrm{~B}_{2} \mathrm{NO}_{6} \mathrm{~S}\left[\mathrm{M}+\mathrm{H}^{+}\right]^{+}$: calculated: 548.3388 , found: 548.3398 . 
(E)-N-(5,5-bis(4,4,5,5-tetramethyl-1,3,2-dioxaborolan-2-yl)-5-(trimethylsilyl)pent-2-en-1-yl)4-methylbenzenesulfonamide (9)

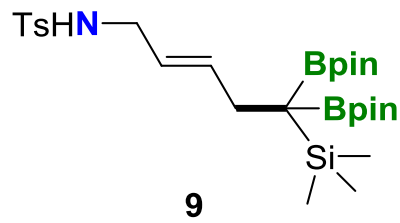

Purified by flash column chromatography (hexane:ethyl acetate $=9: 1$ ) yielded $9(25 \%, 56 \mathrm{mg}$ ) as a white solid.

${ }^{1} \mathrm{H}$ NMR $\left(\mathrm{CDCl}_{3}, 400 \mathrm{MHz}\right) \delta 7.69(\mathrm{~d}, \mathrm{~J}=8.3 \mathrm{~Hz}, 2 \mathrm{H}), 7.26(\mathrm{~d}, \mathrm{~J}=7.6 \mathrm{~Hz}, 2 \mathrm{H}), 5.78-5.61(\mathrm{~m}, 1 \mathrm{H})$, $5.34-5.16(\mathrm{~m}, 1 \mathrm{H}), 4.19(\mathrm{t}, \mathrm{J}=5.9 \mathrm{~Hz}, 1 \mathrm{H}), 3.43(\mathrm{td}, \mathrm{J}=6.2,1.2 \mathrm{~Hz}, 2 \mathrm{H}), 2.38(\mathrm{~s}, 3 \mathrm{H}), 2.21(\mathrm{dd}, \mathrm{J}$ $=7.0,1.4 \mathrm{~Hz}, 2 \mathrm{H}), 1.13(\mathrm{~s}, 24 \mathrm{H}), 0.01(\mathrm{~s}, 9 \mathrm{H})$.

${ }^{13} \mathrm{C} \mathrm{NMR}\left(\mathrm{CDCl}_{3}, 100 \mathrm{MHz}\right) \delta 144.05,138.65,137.92,130.45,127.94,124.14,83.31,46.33,31.36$, $25.71,25.53,22.29,0.00$.

${ }^{11} \mathbf{B}$ NMR $\left(\mathrm{CDCl}_{3}, 128.3 \mathrm{MHz}\right) \delta 33.17$.

HRMS (ESI) for $\mathrm{C}_{27} \mathrm{H}_{48} \mathrm{~B}_{2} \mathrm{NO}_{6} \mathrm{SSi}\left[\mathrm{M}+\mathrm{H}^{+}\right]^{+}$: calculated: 564.3158 , found: 564.3147 .

$\mathrm{N}$-(5,5-bis(4,4,5,5-tetramethyl-1,3,2-dioxaborolan-2-yl)-5-(trimethylsilyl)pent-1-en-3-yl)-4methylbenzenesulfonamide (10)

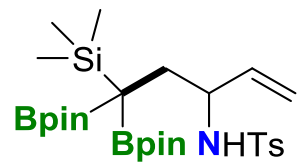

10

Purified by flash column chromatography (hexane:ethyl acetate $=9: 1$ ) yielded 10 (56\%, $126 \mathrm{mg}$ ) as a white solid.

${ }^{1} \mathrm{H}$ NMR $\left(\mathrm{CDCl}_{3}, 400 \mathrm{MHz}\right) \delta 7.60(\mathrm{~d}, \mathrm{~J}=8.3 \mathrm{~Hz}, 2 \mathrm{H}), 7.13(\mathrm{~d}, \mathrm{~J}=8.7 \mathrm{~Hz}, 2 \mathrm{H}), 6.33(\mathrm{~d}, \mathrm{~J}=5.6 \mathrm{~Hz}$, $1 \mathrm{H}), 5.34$ (ddd, J = 17.1, 10.1, 7.9 Hz, 1H), $4.96-4.84(\mathrm{~m}, 1 \mathrm{H}), 4.75-4.64(\mathrm{~m}, 1 \mathrm{H}), 4.22-4.12$ (m, 1H), $2.30(\mathrm{~s}, 3 \mathrm{H}), 1.73-1.54(\mathrm{~m}, 2 \mathrm{H}), 1.27(\mathrm{~s}, 6 \mathrm{H}), 1.25(\mathrm{~s}, 6 \mathrm{H}), 1.10(\mathrm{~s}, 6 \mathrm{H}), 1.07(\mathrm{~s}, 6 \mathrm{H}), 0.01$ $(\mathrm{s}, 9 \mathrm{H})$.

${ }^{13} \mathrm{C} \mathrm{NMR}\left(\mathrm{CDCl}_{3}, 100 \mathrm{MHz}\right) \delta 143.54,141.33,140.90,130.35,128.63,116.45,84.73,84.19,59.13$, $35.07,26.99,26.64,26.10,25.41,22.74,0.01$.

${ }^{11} \mathbf{B}$ NMR $\left(\mathrm{CDCl}_{3}, 128.3 \mathrm{MHz}\right) \delta 32,99$.

HRMS (ESI) for $\mathrm{C}_{27} \mathrm{H}_{48} \mathrm{~B}_{2} \mathrm{NO}_{6} \mathrm{SSi}\left[\mathrm{M}+\mathrm{H}^{+}\right]^{+}$: calculated: 564.3158 , found: 564.3132 . 
4-methyl-N-(5-(tetrahydro-2H-pyran-4-yl)-5,5-bis(4,4,5,5-tetramethyl-1,3,2-dioxaborolan-2-

yl)pent-1-en-3-yl)benzenesulfonamide (12)

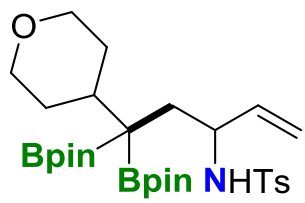

12

Purified by flash column chromatography (hexane:ethyl acetate = 9:1) yielded $12(63 \%, 145 \mathrm{mg})$ as a white solid.

${ }^{1} \mathrm{H} \mathrm{NMR}\left(\mathrm{CDCl}_{3}, 400 \mathrm{MHz}\right) \delta 7.62(\mathrm{~d}, \mathrm{~J}=8.3 \mathrm{~Hz}, 2 \mathrm{H}), 7.17$ (d, J = $\left.8.0 \mathrm{~Hz}, 2 \mathrm{H}\right), 6.05$ (d, J = $5.4 \mathrm{~Hz}$, $1 \mathrm{H}), 5.39(\mathrm{~m}, 1 \mathrm{H}), 4.93(\mathrm{~d}, \mathrm{~J}=17.1 \mathrm{~Hz}, 1 \mathrm{H}), 4.76(\mathrm{~d}, \mathrm{~J}=10.1 \mathrm{~Hz}, 1 \mathrm{H}), 3.82(\mathrm{~m}, 2 \mathrm{H}), 3.19(\mathrm{qd}, \mathrm{J}=$ 11.4, $2.8 \mathrm{~Hz}, 2 \mathrm{H}), 2.34(\mathrm{~s}, 3 \mathrm{H}), 1.87-1.33(\mathrm{~m}, 7 \mathrm{H}), 1.21(\mathrm{~s}, 6 \mathrm{H}), 1.19(\mathrm{~s}, 6 \mathrm{H}), 1.17(\mathrm{~s}, 6 \mathrm{H}), 1.15$ (s, $6 \mathrm{H})$.

${ }^{13} \mathrm{C} \mathrm{NMR}\left(\mathrm{CDCl}_{3}, 100 \mathrm{MHz}\right) \delta 142.64,139.94,138.90,129.16,127.51,115.29,83.68,83.57,68.67$, $68.64,55.64,37.60,35.09,31.11,30.83,25.17,24.89,24.85,24.61,21.47$.

${ }^{11} \mathrm{~B}$ NMR $\left(\mathrm{CDCl}_{3}, 128.3 \mathrm{MHz}\right) \delta 33.82$.

HRMS (ESI) for $\mathrm{C}_{29} \mathrm{H}_{47} \mathrm{~B}_{2} \mathrm{NO}_{7} \mathrm{SNa}\left[\mathrm{M}+\mathrm{Na}^{+}\right]^{+}$: calculated: 598.3157 , found: 598.3162 .

\section{4-methyl-N-(2-((tetrahydro-2H-pyran-4-yl)bis(4,4,5,5-tetramethyl-1,3,2-dioxaborolan-2-}

yl)methyl)but-3-en-1-yl)benzenesulfonamide (13)

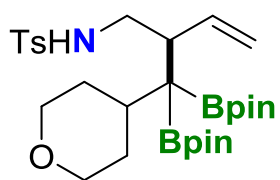

13

Purified by flash column chromatography (hexane:ethyl acetate $=9: 1)$ yielded $\mathbf{1 3}(36 \%, 83 \mathrm{mg}$ ) as a white solid.

${ }^{1} \mathrm{H}$ NMR $\left(\mathrm{CDCl}_{3}, 400 \mathrm{MHz}\right) \delta 7.69(\mathrm{~d}, \mathrm{~J}=8.3 \mathrm{~Hz}, 2 \mathrm{H}), 7.21(\mathrm{~d}, \mathrm{~J}=8.0 \mathrm{~Hz}, 2 \mathrm{H}), 5.50(\mathrm{dt}, \mathrm{J}=17.1,10.1$ $\mathrm{Hz}, 1 \mathrm{H}), 5.00(\mathrm{dd}, \mathrm{J}=10.1,1.9 \mathrm{~Hz}, 1 \mathrm{H}), 4.82(\mathrm{dd}, \mathrm{J}=17.1,1.9 \mathrm{~Hz}, 1 \mathrm{H}), 4.57-4.50(\mathrm{~m}, 1 \mathrm{H}), 3.86-$ $3.72(\mathrm{~m}, 2 \mathrm{H}), 3.34-3.28(\mathrm{~m},, 2 \mathrm{H}), 3.27-3.10(\mathrm{~m}, 2 \mathrm{H}), 2.75-2.64(\mathrm{~m}, 1 \mathrm{H}), 2.38-2.26(\mathrm{~m}, 4 \mathrm{H})$, $1.75-1.33(\mathrm{~m}, 4 \mathrm{H}), 1.14(\mathrm{~s}, 6 \mathrm{H}), 1.13(\mathrm{~s}, 6 \mathrm{H}), 1.08(\mathrm{~s}, 12 \mathrm{H})$.

${ }^{13} \mathrm{C} \mathrm{NMR}\left(\mathrm{CDCl}_{3}, 100 \mathrm{MHz}\right) \delta$ 142.95, 139.35, 137.58, 129.42, 127.37, 118.03, 83.20, 83.11, 68.83, $68.71,45.62,44.80,37.13,31.44,30.51,24.95,24.88,24.85,24.82,24.74,21.45$.

${ }^{11}$ B NMR $\left(\mathrm{CDCl}_{3}, 128.3 \mathrm{MHz}\right) \delta 34,64$.

HRMS (ESI) for $\mathrm{C}_{29} \mathrm{H}_{47} \mathrm{~B}_{2} \mathrm{NO}_{7} \mathrm{SNa}$ [M+Na+] ${ }^{+}$: calculated: 598.3157 , found: 598.3165 . 
(E)-4-methyl-N-(1-phenyl-5,5-bis(4,4,5,5-tetramethyl-1,3,2-dioxaborolan-2-yl)-5-

(trimethylsilyl)pent-1-en-3-yl)benzenesulfonamide (15)

$\left(\mathrm{CH}_{3}\right)_{3} \mathrm{Si} \overbrace{\text { Bpin } \mathrm{NHTs}^{2}}^{\mathrm{Ph}}$

15

Purified by flash column chromatography (hexane:ethyl acetate $=9: 1)$ yielded 15 (12\%, $31 \mathrm{mg})$ as a white solid.

${ }^{1} \mathrm{H} \mathrm{NMR}\left(\mathrm{CDCl}_{3}, 400 \mathrm{MHz}\right) \delta 7.50(\mathrm{~d}, \mathrm{~J}=8.3 \mathrm{~Hz}, 2 \mathrm{H}), 7.15-7.01(\mathrm{~m}, 3 \mathrm{H}), 6.97-6.85(\mathrm{~m}, 4 \mathrm{H}), 6.35$ (d, J = 6.2 Hz, 1H), $6.15(\mathrm{~d}, \mathrm{~J}=15.8 \mathrm{~Hz}, 1 \mathrm{H}), 5.40(\mathrm{dd}, \mathrm{J}=15.8,8.6 \mathrm{~Hz}, 1 \mathrm{H}), 4.43-4.32(\mathrm{~m}, 1 \mathrm{H})$, $2.13(\mathrm{~s}, 3 \mathrm{H}), 1.73-1.60(\mathrm{~m}, 2 \mathrm{H}), 1.25(\mathrm{~s}, 6 \mathrm{H}), 1.20(\mathrm{~s}, 6 \mathrm{H}), 1.19(\mathrm{~s}, 6 \mathrm{H}), 1.16(\mathrm{~s}, 6 \mathrm{H}), 0.01(\mathrm{~s}, 9 \mathrm{H})$. ${ }^{13} \mathrm{C}$ NMR $\left(\mathrm{CDCl}_{3}, 100 \mathrm{MHz}\right) \delta 143.39,141.19,138.08,132.75,131.74,130.34,129.41,128.58$, $128.47,127.58,84.83,84.27,58.56,34.88,27.01,26.66,26.11,25.37,22.55,0.01$.

${ }^{11}$ B NMR $\left(\mathrm{CDCl}_{3}, 128.3 \mathrm{MHz}\right) \delta 33.40$

HRMS (ESI) for $\mathrm{C}_{33} \mathrm{H}_{52} \mathrm{~B}_{2} \mathrm{NO}_{6}$ SSi [M+H'] ${ }^{+}$: calculated: 640.3471 , found: 640.3475 .

(E)-N-(2-(bis(4,4,5,5-tetramethyl-1,3,2-dioxaborolan-2-yl)(trimethylsilyl)methyl)-4phenylbut-3-en-1-yl)-4-methylbenzenesulfonamide (16)<smiles>CNCC(/C=C/P)C([Si])([Si])c1ccccc1</smiles>

16

Purified by flash column chromatography (hexane:ethyl acetate $=9: 1)$ yielded $\mathbf{1 6}(23 \%, 59 \mathrm{mg})$ as a white solid.

${ }^{1} \mathrm{H}$ NMR $\left(\mathrm{CDCl}_{3}, 400 \mathrm{MHz}\right) \delta 7.79-7.71(\mathrm{~m}, 2 \mathrm{H}), 7.33-7.12(\mathrm{~m}, 7 \mathrm{H}), 6.06(\mathrm{dd}, \mathrm{J}=16.0,9.8 \mathrm{~Hz}$, $1 \mathrm{H}), 5.96(\mathrm{~d}, \mathrm{~J}=16.0 \mathrm{~Hz}, 1 \mathrm{H}), 4.69(\mathrm{dd}, \mathrm{J}=8.3,3.6 \mathrm{~Hz}, 1 \mathrm{H}), 3.39-3.22(\mathrm{~m}, 2 \mathrm{H}), 2.59-2.49(\mathrm{~m}$, $1 \mathrm{H}), 2.38(\mathrm{~s}, 3 \mathrm{H}), 1.25(\mathrm{~s}, 6 \mathrm{H}), 1.21(\mathrm{~s}, 6 \mathrm{H}), 1.18(\mathrm{~s}, 12 \mathrm{H}), 0.01(\mathrm{~s}, 9 \mathrm{H})$.

${ }^{13} \mathrm{C}$ NMR $\left(\mathrm{CDCl}_{3}, 100 \mathrm{MHz}\right) \delta 142.66,137.90,136.87,132.57,131.56,129.22,128.35,127.20$, $127.05,125.93,82.78,82.48,47.27,43.34,25.26,25.07,24.57,24.28,21.28,0.01$.

${ }^{11}$ B NMR $\left(\mathrm{CDCl}_{3}, 128.3 \mathrm{MHz}\right) \delta 34.21$.

HRMS (ESI) for $\mathrm{C}_{33} \mathrm{H}_{52} \mathrm{~B}_{2} \mathrm{NO}_{6} \mathrm{SSi}\left[\mathrm{M}+\mathrm{H}^{+}\right]^{+}$: calculated: 640.3471 , found: 640.3473 
(E)-4-methyl-N-(4-phenyl-1,1-bis(4,4,5,5-tetramethyl-1,3,2-dioxaborolan-2-yl)-1-

(trimethylsilyl)hex-4-en-3-yl)benzenesulfonamide (18)<smiles>[10BH2]C(CC([10BH2])([10BH2])[13CH3])/C(=C/C)c1ccccc1</smiles>

18

Purified by flash column chromatography (hexane:ethyl acetate $=9: 1$ ) yielded $\mathbf{1 8}(9 \%, 24 \mathrm{mg}$ ) as a white solid.

${ }^{1} \mathrm{H}$ NMR $\left(\mathrm{CDCl}_{3}, 400 \mathrm{MHz}\right) \delta 7.73-7.65(\mathrm{~m}, 2 \mathrm{H}), 7.29-7.13(\mathrm{~m}, 5 \mathrm{H}), 7.09-7.00(\mathrm{~m}, 2 \mathrm{H}), 6.60$ $(\mathrm{d}, \mathrm{J}=4.9 \mathrm{~Hz}, 1 \mathrm{H}), 5.34-5.24(\mathrm{~m}, 1 \mathrm{H}), 4.74-4.65(\mathrm{~m}, 1 \mathrm{H}), 2.37(\mathrm{~s}, 3 \mathrm{H}), 1.75$ (dd, J = 14.5, $2.8 \mathrm{~Hz}$, 1H), $1.54(\mathrm{dd}, \mathrm{J}=14.5,11.0 \mathrm{~Hz}, 1 \mathrm{H}), 1.34(\mathrm{~s}, 6 \mathrm{H}), 1.27(\mathrm{~s}, 6 \mathrm{H}), 1.24(\mathrm{~s}, 6 \mathrm{H}), 1.19(\mathrm{~s}, 6 \mathrm{H}), 0.01(\mathrm{~s}$, $9 \mathrm{H})$.

${ }^{13} \mathrm{C}$ NMR $\left(\mathrm{CDCl}_{3}, 100 \mathrm{MHz}\right.$ 143.39, 141.19, 138.08, 132.75, 131.74, 130.34, 129.41, 128.58, $128.47,127.58,84.83,84.27,78.64,78.33$, 78.01, 58.56, 34.88, 27.01, 26.66, 26.11, 25.37, 22.55, $-0.01)$

${ }^{11} \mathrm{~B}$ NMR $\left(\mathrm{CDCl}_{3}, 128.3 \mathrm{MHz}\right) \delta 32,90$.

HRMS (ESI) for $\mathrm{C}_{34} \mathrm{H}_{54} \mathrm{~B}_{2} \mathrm{NO}_{6}$ SSi [M+H+ $]^{+}$: calculated: 654.3627 , found: 654.3631 .

(Z)-N-(2-(bis(4,4,5,5-tetramethyl-1,3,2-dioxaborolan-2-yl)(trimethylsilyl)methyl)-3phenylpent-3-en-1-yl)-4-methylbenzenesulfonamide (19)

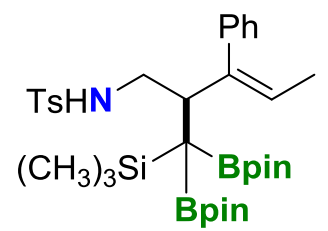

19

Purified by flash column chromatography (hexane:ethyl acetate = 9:1) yielded 19 (12\%, $31 \mathrm{mg})$ as a white solid.

${ }^{1} \mathrm{H} \mathrm{NMR}\left(\mathrm{CDCl}_{3}, 400 \mathrm{MHz}\right) \delta 7.60(\mathrm{~d}, \mathrm{~J}=8.3 \mathrm{~Hz}, 2 \mathrm{H}), 7.30-7.08(\mathrm{~m}, 5 \mathrm{H}), 7.08-6.97(\mathrm{~m}, 2 \mathrm{H}), 6.57$ $(\mathrm{dd}, \mathrm{J}=8.0,2.4 \mathrm{~Hz}, 1 \mathrm{H}), 5.55(\mathrm{qd}, \mathrm{J}=6.7,1.3 \mathrm{~Hz}, 1 \mathrm{H}), 3.10-2.98(\mathrm{~m}, 2 \mathrm{H}), 2.97-2.80(\mathrm{~m}, 2 \mathrm{H})$, $2.36(\mathrm{~s}, 3 \mathrm{H}), 1.39$ (dd, J = 6.7, 1.2 Hz, 3H), $1.22(\mathrm{br}, 24 \mathrm{H}), 0.01(\mathrm{~s}, 9 \mathrm{H})$.

${ }^{13} \mathrm{C}$ NMR $\left(\mathrm{CDCl}_{3}, 100 \mathrm{MHz}\right) \delta 142.00,141.96,140.96,137.08,128.81,128.45,127.35,126.70$, $125.86,122.77,82.76,82.39,45.71,44.91,24.91,24.72,24.62,24.28,20.94,14.06,0.01$.

${ }^{11} \mathrm{~B}$ NMR $\left(\mathrm{CDCl}_{3}, 128.3 \mathrm{MHz}\right) \delta 34.68$.

HRMS (ESI) for $\mathrm{C}_{34} \mathrm{H}_{54} \mathrm{~B}_{2} \mathrm{NO}_{6}$ SSi [M+H+ $]^{+}$: calculated: 654.3627 , found: 654.3620 . 
(E)-4-methyl-N-(2-methyl-5,5-bis(4,4,5,5-tetramethyl-1,3,2-dioxaborolan-2-yl)-5-

(trimethylsilyl)pent-2-en-1-yl)benzenesulfonamide (21)

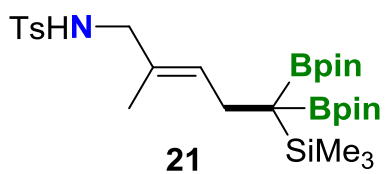

Purified by flash column chromatography (hexane:ethyl acetate $=9: 1)$ yielded $\mathbf{2 1}(93 \%, 215 \mathrm{mg}$ ) as a white solid.

${ }^{1} \mathrm{H}$ NMR $\left(\mathrm{CDCl}_{3}, 400 \mathrm{MHz}\right) \delta 7.69(\mathrm{~d}, \mathrm{~J}=8.3 \mathrm{~Hz}, 2 \mathrm{H}), 7.24(\mathrm{~d}, \mathrm{~J}=7.7 \mathrm{~Hz}, 2 \mathrm{H}), 5.41-5.31(\mathrm{~m}, 1 \mathrm{H})$, $4.12(\mathrm{t}, \mathrm{J}=6.0 \mathrm{~Hz}, 1 \mathrm{H}), 3.34(\mathrm{~d}, \mathrm{~J}=6.0 \mathrm{~Hz}, 2 \mathrm{H}), 2.37(\mathrm{~s}, 3 \mathrm{H}), 2.17(\mathrm{~d}, \mathrm{~J}=7.0 \mathrm{~Hz}, 2 \mathrm{H}), 1.51(\mathrm{~s}, 3 \mathrm{H})$, $1.11(\mathrm{~s}, 24 \mathrm{H}), 0.01(\mathrm{~s}, 9 \mathrm{H})$.

${ }^{13} \mathrm{C} \mathrm{NMR}\left(\mathrm{CDCl}_{3}, 100 \mathrm{MHz}\right) \delta 144.06,137.98,133.37,130.48,128.80,128.07,83.38,52.72,26.93$, $25.77,25.56,22.41,15.46,0.00$.

${ }^{11} \mathrm{~B}$ NMR $\left(\mathrm{CDCl}_{3}, 128.3 \mathrm{MHz}\right) \delta 32.80$.

HRMS (ESI) for $\mathrm{C}_{28} \mathrm{H}_{49} \mathrm{~B}_{2} \mathrm{NO}_{6} \mathrm{SSiNa}\left[\mathrm{M}+\mathrm{Na}^{+}\right]^{+}$: calculated: 600.3133 , found: 600.3134 .

(E)-4-methyl-N-(2,6,6-trimethyl-5,5-bis(4,4,5,5-tetramethyl-1,3,2-dioxaborolan-2-yl)hept-2en-1-yl)benzenesulfonamide (22)

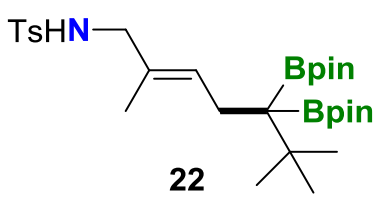

Purified by flash column chromatography (hexane:ethyl acetate $=9: 1$ ) yielded $\mathbf{2 2}(96 \%, 216 \mathrm{mg}$ ) as a white-yellowish solid.

${ }^{1} \mathrm{H} \mathrm{NMR}\left(\mathrm{CDCl}_{3}, 400 \mathrm{MHz}\right) \delta 7.67(\mathrm{~d}, \mathrm{~J}=8.3 \mathrm{~Hz}, 2 \mathrm{H}), 7.22(\mathrm{~d}, \mathrm{~J}=8.0 \mathrm{~Hz}, 2 \mathrm{H}), 5.41$ (tq, J = 6.9, 1.3 $\mathrm{Hz}, 1 \mathrm{H}), 4.24(\mathrm{t}, \mathrm{J}=5.9 \mathrm{~Hz}, 1 \mathrm{H}), 3.33(\mathrm{~d}, \mathrm{~J}=5.3 \mathrm{~Hz}, 2 \mathrm{H}), 2.34(\mathrm{~s}, 3 \mathrm{H}), 2.16(\mathrm{~d}, \mathrm{~J}=6.5 \mathrm{~Hz}, 2 \mathrm{H}), 1.47$ (d, J = $1.4 \mathrm{~Hz}, 3 \mathrm{H}), 1.11(\mathrm{~s}, 24 \mathrm{H}), 0.93(\mathrm{~s}, 9 \mathrm{H})$.

${ }^{13} \mathrm{C} \mathrm{NMR}\left(\mathrm{CDCl}_{3}, 100 \mathrm{MHz}\right) \delta 143.12,137.23,132.07,129.56,127.74,127.22,82.54,51.84,33.95$, $29.62,28.15,24.88,24.75,21.51,14.51$.

${ }^{11} \mathrm{~B}$ NMR $\left(\mathrm{CDCl}_{3}, 128.3 \mathrm{MHz}\right) \delta 33.37$.

HRMS (ESI) for $\mathrm{C}_{29} \mathrm{H}_{49} \mathrm{~B}_{2} \mathrm{NO}_{6} \mathrm{SNa}\left[\mathrm{M}+\mathrm{Na}^{+}\right]^{+}$: calculated: 584.3364 , found: 584.3375 
(E)-4-methyl-N-(2-methyl-5,5-bis(4,4,5,5-tetramethyl-1,3,2-dioxaborolan-2-yl)hex-2-en-1yl)benzenesulfonamide (23)

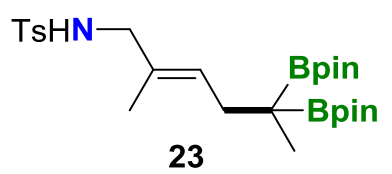

Purified by flash column chromatography (hexane:ethyl acetate $=9: 1$ ) yielded $\mathbf{2 3}(98 \%, 204 \mathrm{mg}$ ) as a white solid.

${ }^{1} \mathrm{H}$ NMR $\left(\mathrm{CDCl}_{3}, 400 \mathrm{MHz}\right) \delta 7.66(\mathrm{~d}, \mathrm{~J}=8.3 \mathrm{~Hz}, 2 \mathrm{H}), 7.22(\mathrm{~d}, \mathrm{~J}=8.4 \mathrm{~Hz}, 2 \mathrm{H}), 5.23(\mathrm{td}, \mathrm{J}=7.4,1.3$ $\mathrm{Hz}, 1 \mathrm{H}), 4.23(\mathrm{~m}, 1 \mathrm{H}), 3.35(\mathrm{~d}, \mathrm{~J}=6.1,2 \mathrm{H}), 2.35(\mathrm{~s}, 3 \mathrm{H}), 2.10(\mathrm{~d}, \mathrm{~J}=7.1 \mathrm{~Hz}, 2 \mathrm{H}), 1.49(\mathrm{~s}, 3 \mathrm{H}), 1.13$ $(\mathrm{s}, 24 \mathrm{H}), 0.88(\mathrm{~s}, 3 \mathrm{H})$.

${ }^{13} \mathrm{C} \mathrm{NMR}\left(\mathrm{CDCl}_{3}, 100 \mathrm{MHz}\right) \delta 143.23,137.14,130.59,129.63,128.69,127.15,83.07,51.56,31.77$, $24.67,24.63,21.51,16.03,14.57$.

${ }^{11} \mathrm{~B}$ NMR $\left(\mathrm{CDCl}_{3}, 128.3 \mathrm{MHz}\right) \delta 34.35$.

HRMS (ESI) for $\mathrm{C}_{26} \mathrm{H}_{43} \mathrm{~B}_{2} \mathrm{NO}_{6} \mathrm{SNa}\left[\mathrm{M}+\mathrm{Na}^{+}\right]^{+}$: calculated: 542.2894 , found: 542.2906 .

(E)-4-methyl-N-(2-methyl-5-(tetrahydro-2H-pyran-4-yl)-5,5-bis(4,4,5,5-tetramethyl-1,3,2dioxaborolan-2-yl)pent-2-en-1-yl)benzenesulfonamide (24)

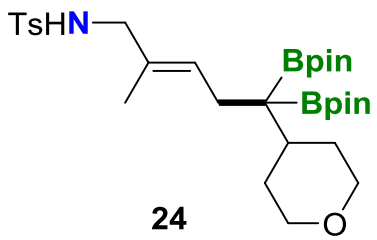

Purified by flash column chromatography (hexane:ethyl acetate $=9: 1$ ) yielded $\mathbf{2 4}(99 \%, 233 \mathrm{mg}$ ) as a white solid.

${ }^{1} \mathrm{H}$ NMR $\left(\mathrm{CDCl}_{3}, 400 \mathrm{MHz}\right) \delta 7.67(\mathrm{~d}, \mathrm{~J}=8.4 \mathrm{~Hz}, 2 \mathrm{H}), 7.23(\mathrm{~d}, \mathrm{~J}=7.8 \mathrm{~Hz}, 2 \mathrm{H}), 5.32(\mathrm{td}, \mathrm{J}=7.0,1.3$ $\mathrm{Hz}, 1 \mathrm{H}), 4.22(\mathrm{t}, \mathrm{J}=6.3 \mathrm{~Hz}, 1 \mathrm{H}), 3.84(\mathrm{dd}, \mathrm{J}=10.7,3.3 \mathrm{~Hz}, 2 \mathrm{H}), 3.35(\mathrm{~d}, \mathrm{~J}=6.1 \mathrm{~Hz}, 2 \mathrm{H}), 3.29(\mathrm{td}, \mathrm{J}$ $=11.4,2.7 \mathrm{~Hz}, 2 \mathrm{H}), 2.36(\mathrm{~s}, 3 \mathrm{H}), 2.20(\mathrm{~d}, \mathrm{~J}=7.1 \mathrm{~Hz}, 2 \mathrm{H}), 1.79(\mathrm{~m}, 1 \mathrm{H}), 1.55-1.39(\mathrm{~m}, 4 \mathrm{H}), 1.35(\mathrm{~s}$, $3 \mathrm{H}), 1.13(\mathrm{~s}, 24 \mathrm{H})$.

${ }^{13} \mathrm{C} \mathrm{NMR}\left(\mathrm{CDCl}_{3}, 100 \mathrm{MHz}\right) \delta 143.24,137.19,129.78,129.63,129.20,127.20,83.04,68.91,51.57$, $37.61,31.38,27.26,24.82,24.77,21.53,14.61$.

${ }^{11}$ B NMR $\left(\mathrm{CDCl}_{3}, 128.3 \mathrm{MHz}\right) \delta 33.40$.

HRMS (ESI) for $\mathrm{C}_{30} \mathrm{H}_{49} \mathrm{~B}_{2} \mathrm{NO}_{7} \mathrm{SNa}\left[\mathrm{M}+\mathrm{Na}^{+}\right]^{+}$: calculated: 612.3313 , found: 612.3314 . 
(E)-4-methyl-N-(2-methyl-7-phenyl-5,5-bis(4,4,5,5-tetramethyl-1,3,2-dioxaborolan-2-yl)hept2-en-1-yl)benzenesulfonamide (26)

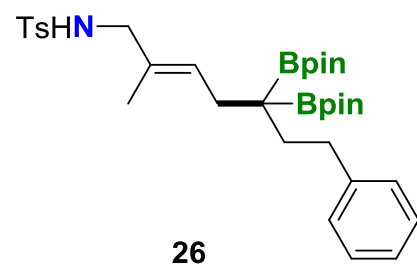

Purified by flash column chromatography (hexane:ethyl acetate $=9: 1)$ yielded $\mathbf{2 6}(50 \%, 122 \mathrm{mg}$ ) as a white solid.

${ }^{1} \mathrm{H}$ NMR $\left(\mathrm{CDCl}_{3}, 400 \mathrm{MHz}\right) \delta 7.65(\mathrm{~d}, \mathrm{~J}=8.4 \mathrm{~Hz}, 2 \mathrm{H}), 7.23-7.13(\mathrm{~m}, 4 \mathrm{H}), 7.11-7.00(\mathrm{~m}, 3 \mathrm{H}), 5.22$ (tq, J = 7.4, 1.4 Hz, 1H), $4.20(\mathrm{t}, \mathrm{J}=6.3 \mathrm{~Hz}, 1 \mathrm{H}), 3.36(\mathrm{~d}, \mathrm{~J}=6.3 \mathrm{~Hz}, 2 \mathrm{H}), 2.48-2.36(\mathrm{~m}, 2 \mathrm{H}), 2.35$ $(\mathrm{s}, 3 \mathrm{H}) 2.33-2.22(\mathrm{~m}, 2 \mathrm{H}), 1.79-1.64(\mathrm{~m}, 2 \mathrm{H}), 1.58(\mathrm{~s}, \mathrm{~J}=1.4 \mathrm{~Hz}, 3 \mathrm{H}), 1.14(\mathrm{~s}, 24 \mathrm{H})$.

${ }^{13} \mathrm{C} \mathrm{NMR}\left(\mathrm{CDCl}_{3}, 100 \mathrm{MHz}\right) \delta 143.48,143.26,137.14,130.50,129.67,128.57,128.44,128.21$, $127.15,125.52,83.20,51.61,33.93,32.44,27.63,24.84,24.70,21.51,14.74$.

${ }^{11} \mathrm{~B}$ NMR $\left(\mathrm{CDCl}_{3}, 128.3 \mathrm{MHz}\right) \delta 34.05$.

HRMS (ESI) for $\mathrm{C}_{33} \mathrm{H}_{49} \mathrm{~B}_{2} \mathrm{NO}_{6} \mathrm{SNa}\left[\mathrm{M}+\mathrm{Na}^{+}\right]^{+}$: calculated: 632.3364 , found: 632.3381 .

(E)-4-methyl-N-(2-methyl-6-phenyl-5,5-bis(4,4,5,5-tetramethyl-1,3,2-dioxaborolan-2-yl)hex2-en-1-yl)benzenesulfonamide (28)

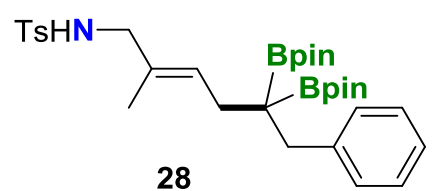

Purified by flash column chromatography (hexane:ethyl acetate $=9: 1)$ yielded $\mathbf{2 8}(75 \%, 179 \mathrm{mg}$ ) as a white solid.

${ }^{1} \mathrm{H} \mathrm{NMR}\left(\mathrm{CDCl}_{3}, 400 \mathrm{MHz}\right) \delta 7.67(\mathrm{~d}, \mathrm{~J}=8.3 \mathrm{~Hz}, 2 \mathrm{H}), 7.23(\mathrm{~d}, \mathrm{~J}=8.3 \mathrm{~Hz}, 2 \mathrm{H}), 7.13-7.03(\mathrm{~m}, 5 \mathrm{H})$, $5.24(\mathrm{tq}, \mathrm{J}=6.8,1.2 \mathrm{~Hz}, 1 \mathrm{H}), 4.13(\mathrm{t}, \mathrm{J}=6.2 \mathrm{~Hz}, 1 \mathrm{H}), 3.35(\mathrm{~d}, \mathrm{~J}=6.2 \mathrm{~Hz} 2 \mathrm{H}), 2.81(\mathrm{~s}, 2 \mathrm{H}), 2.34(\mathrm{~s}$, $3 \mathrm{H}), 2.10(\mathrm{~d}, \mathrm{~J}=6.7 \mathrm{~Hz}, 2 \mathrm{H}), 1.38(\mathrm{~d}, \mathrm{~J}=1.3 \mathrm{~Hz}, 3 \mathrm{H}), 1.14(\mathrm{~s}, 12 \mathrm{H}), 1.13(\mathrm{~s}, 12 \mathrm{H})$.

${ }^{13} \mathrm{C}$ NMR $\left(\mathrm{CDCl}_{3}, 100 \mathrm{MHz}\right) \delta 143.28,141.53,137.17,130.41,129.76,129.67,128.88,127.74$, $127.16,125.55,83.37,51.50,35.34,27.40,24.95,24.73,21.51,14.73$.

${ }^{11} \mathbf{B}$ NMR $\left(\mathrm{CDCl}_{3}, 128.3 \mathrm{MHz}\right) \delta 32.91$.

HRMS (ESI) for $\mathrm{C}_{32} \mathrm{H}_{47} \mathrm{~B}_{2} \mathrm{NO}_{6} \mathrm{SNa}\left[\mathrm{M}+\mathrm{Na}^{+}\right]^{+}$: calculated: 618.3207 , found: 618.3213 . 
(E)-4-methyl-N-(2-methyl-5-(4,4,5,5-tetramethyl-1,3,2-dioxaborolan-2-yl)-5-(p-tolyl)pent-2en-1-yl)benzenesulfonamide (30)<smiles>C/C(=C\CC(c1ccccc1)(c1ccccc1)c1ccccc1)CN</smiles>

Purified by flash column chromatography (hexane:ethyl acetate $=9: 1$ ) yielded 30 (65\%, $122 \mathrm{mg}$ ) as a white solid.

${ }^{1} \mathrm{H}$ NMR $\left(\mathrm{CDCl}_{3}, 400 \mathrm{MHz}\right) \delta 7.63(\mathrm{~d}, \mathrm{~J}=8.4 \mathrm{~Hz}, 2 \mathrm{H}), 7.22(\mathrm{~d}, \mathrm{~J}=7.9 \mathrm{~Hz}, 2 \mathrm{H}), 6.97(\mathrm{~s}, 4 \mathrm{H}), 5.21-$ $5.14(\mathrm{~m}, 1 \mathrm{H}), 4.04(\mathrm{t}, \mathrm{J}=6.5 \mathrm{~Hz}, 1 \mathrm{H}), 3.31(\mathrm{~d}, \mathrm{~J}=6.3 \mathrm{~Hz}, 2 \mathrm{H}), 2.34(\mathrm{~s}, 3 \mathrm{H}), 2.33-2.31(\mathrm{~m}, 1 \mathrm{H}), 2.21$ (s, 3H), $2.20-2.11(\mathrm{~m}, 2 \mathrm{H}), 1.42(\mathrm{~s}, 3 \mathrm{H}), 1.11(\mathrm{~s}, 6 \mathrm{H}), 1.09(\mathrm{~s}, 6 \mathrm{H})$.

${ }^{13} \mathrm{C}$ NMR $\left(\mathrm{CDCl}_{3}, 100 \mathrm{MHz}\right) \delta 143.28,139.35,137.14,134.79,130.78,129.64,129.04,128.54$, $128.28,127.14,83.41,51.30,30.91,24.64,24.62,21.53,20.99,14.38$.

${ }^{11} \mathrm{~B}$ NMR $\left(\mathrm{CDCl}_{3}, 128.3 \mathrm{MHz}\right) \delta 32.22$.

HRMS (ESI) for $\mathrm{C}_{26} \mathrm{H}_{37} \mathrm{BNO}_{4} \mathrm{~S}$ [M+H+ ${ }^{+}$: calculated: 470.2536 , found: 470.2548 .

(E)-6,6-dimethyl-5,5-bis(4,4,5,5-tetramethyl-1,3,2-dioxaborolan-2-yl)hept-2-en-1-ol (33)

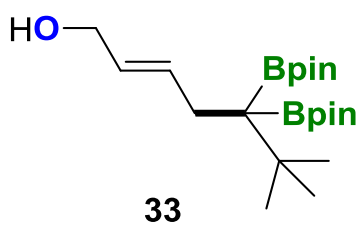

Purified by flash column chromatography (hexane:ethyl acetate $=9: 1$ ) yielded 33 (90\%, $142 \mathrm{mg}$ ) as a white solid.

${ }^{1} \mathrm{H}$ NMR $\left(\mathrm{CDCl}_{3}, 400 \mathrm{MHz}\right) \delta 5.93-5.77(\mathrm{~m}, 1 \mathrm{H}), 5.68-5.51(\mathrm{~m}, 1 \mathrm{H}), 3.97(\mathrm{~d}, \mathrm{~J}=6.2 \mathrm{~Hz}, 2 \mathrm{H}), 2.31$ (dd, J = 7.1, 1.4 Hz, 2H), $1.16(\mathrm{~s}, 12 \mathrm{H}), 1.12(\mathrm{~s}, 12 \mathrm{H}), 0.97(\mathrm{~s}, 9 \mathrm{H})$.

${ }^{13} \mathrm{C} \mathrm{NMR}\left(\mathrm{CDCl}_{3}, 100 \mathrm{MHz}\right) \delta 136.21,128.49,82.55,64.26,34.05,32.96,29.65,24.94,24.79$.

${ }^{11} \mathbf{B}$ NMR $\left(\mathrm{CDCl}_{3}, 128.3 \mathrm{MHz}\right) \delta 33.87$.

HRMS (ESI) for $\mathrm{C}_{21} \mathrm{H}_{44} \mathrm{NB}_{2} \mathrm{O}_{5}\left[\mathrm{M}+\mathrm{NH}_{4}^{+}\right]^{+}$: calculated: 412.3405 , found: 412.3409 . 
(E)-5,5-bis(4,4,5,5-tetramethyl-1,3,2-dioxaborolan-2-yl)-5-(trimethylsilyl)pent-2-en-1-ol (34)

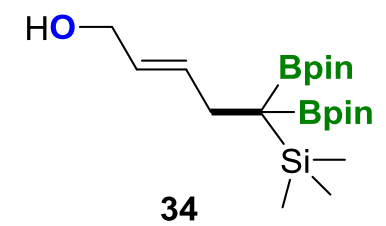

Purified by flash column chromatography (hexane:ethyl acetate $=9: 1)$ yielded $\mathbf{3 4}(53 \%, 87 \mathrm{mg})$ as a white solid.

${ }^{1} \mathrm{H}$ NMR $\left(\mathrm{CDCl}_{3}, 400 \mathrm{MHz}\right) \delta 5.85-5.66(\mathrm{~m}, 1 \mathrm{H}), 5.66-5.46(\mathrm{~m}, 1 \mathrm{H}), 3.95(\mathrm{~d}, \mathrm{~J}=6.2 \mathrm{~Hz}, 2 \mathrm{H}), 2.28$ (dd, J = 6.9, 1.3 Hz, 2H), $1.15(\mathrm{~s}, 12 \mathrm{H}), 1.07(\mathrm{~s}, 12 \mathrm{H}), 0.01(\mathrm{~s}, 9 \mathrm{H})$.

${ }^{13} \mathrm{C} \mathrm{NMR}\left(\mathrm{CDCl}_{3}, 100 \mathrm{MHz}\right) \delta 136.71,129.05,83.21,64.89,31.40,25.66,25.47,0.01$.

${ }^{11} \mathbf{B}$ NMR $\left(\mathrm{CDCl}_{3}, 128.3 \mathrm{MHz}\right) \delta 33.39$.

HRMS (ESI) for $\mathrm{C}_{20} \mathrm{H}_{44} \mathrm{~B}_{2} \mathrm{NO5Si}\left[\mathrm{M}+\mathrm{NH}_{4}^{+}\right]^{+}$: calculated: 428.31758 , found: 428.3168 .

$\mathrm{N}$-(2-(1,1-bis(4,4,5,5-tetramethyl-1,3,2-dioxaborolan-2-yl)ethyl)cyclohex-3-en-1-yl)-4methylbenzenesulfonamide (36)<smiles>CC(Br)(Br)c1ccccc1</smiles>

Purified by flash column chromatography (hexane:ethyl acetate $=9: 1$ ) yielded $36(98 \%, 208 \mathrm{mg}$ ) as a white solid.

${ }^{1} \mathrm{H}$ NMR $\left(\mathrm{CDCl}_{3}, 400 \mathrm{MHz}\right) \delta 7.69(\mathrm{~d}, \mathrm{~J}=8.3 \mathrm{~Hz}, 2 \mathrm{H}), 7.31-7.11(\mathrm{~m}, 2 \mathrm{H}), 5.54(\mathrm{dq}, \mathrm{J}=10.0,3.3$ $\mathrm{Hz}, 1 \mathrm{H}), 5.36(\mathrm{~d}, \mathrm{~J}=7.3 \mathrm{~Hz}, 1 \mathrm{H}), 5.22(\mathrm{dq}, \mathrm{J}=10.1,2.1 \mathrm{~Hz}, 1 \mathrm{H}), 3.28(\mathrm{~m}, 1 \mathrm{H}), 2.74-2.56(\mathrm{~m}, 1 \mathrm{H})$, $2.33(\mathrm{~s}, 3 \mathrm{H}), 1.90-1.77(\mathrm{~m}, 2 \mathrm{H}), 1.72-1.59(\mathrm{~m}, 1 \mathrm{H}), 1.35-1.33(\mathrm{~m}, 1 \mathrm{H}), 1.32(\mathrm{~s}, 6 \mathrm{H}), 1.31(\mathrm{~s}$, $6 \mathrm{H}), 1.15(\mathrm{~s}, 6 \mathrm{H}), 1.14(\mathrm{~s}, 6 \mathrm{H}), 0.83(\mathrm{~s}, 3 \mathrm{H})$.

${ }^{13} \mathrm{C}$ NMR $\left(\mathrm{CDCl}_{3}, 100 \mathrm{MHz}\right) \delta$ 142.86, 139.50, 130.96, 129.49, 128.39, 127.07, 83.97, 83.47, 52.43, $44.02,29.38,25.63,25.11,24.56,24.53,24.49,21.49,10.76$.

${ }^{11}$ B NMR $\left(\mathrm{CDCl}_{3}, 128.3 \mathrm{MHz}\right) \delta 33.31$.

HRMS (ESI) for $\mathrm{C}_{27} \mathrm{H}_{43} \mathrm{~B}_{2} \mathrm{NNaO}_{6} \mathrm{~S}$ [M+Na+] ${ }^{+}$: calculated: 554.2894 , found: 554.2904 
N-(2-(2,2-dimethyl-1,1-bis(4,4,5,5-tetramethyl-1,3,2-dioxaborolan-2-yl)propyl)cyclohex-3-en1-yl)-4-methylbenzenesulfonamide (37)<smiles></smiles>

Purified by flash column chromatography (hexane:ethyl acetate $=9: 1$ ) yielded $\mathbf{3 7}(98 \%, 225 \mathrm{mg}$ ) as a white-yellowish solid.

${ }^{1} \mathrm{H}$ NMR $\left(\mathrm{CDCl}_{3}, 400 \mathrm{MHz}\right) \delta 7.71(\mathrm{~d}, \mathrm{~J}=8.3 \mathrm{~Hz}, 2 \mathrm{H}), 7.30-7.03(\mathrm{~m}, 2 \mathrm{H}), 5.50(\mathrm{~d}, 2 \mathrm{H}), 5.29(\mathrm{~d}, \mathrm{~J}=$ $8.0 \mathrm{~Hz}, 1 \mathrm{H}), 4.29(\mathrm{~m}, 1 \mathrm{H}), 2.32(\mathrm{~s}, 3 \mathrm{H}), 2.28-2.18(\mathrm{~m}, 1 \mathrm{H}), 1.91(\mathrm{~m}, 3 \mathrm{H}), 1.44-1.25(\mathrm{~m}, 1 \mathrm{H}), 1.13$ $(\mathrm{s}, 24 \mathrm{H}), 0.88$ (s. 9H).

${ }^{13} \mathrm{C}$ NMR $\left(\mathrm{CDCl}_{3}, 100 \mathrm{MHz}\right) \delta 142.47,139.61,130.68,129.36,127.57,127.03,82.71,82.68,51.57$, $42.61,35.36,30.13,25.37,25.01,24.90,24.88,24.59,21.69,21.44$.

${ }^{11} \mathrm{~B}$ NMR $\left(\mathrm{CDCl}_{3}, 128.3 \mathrm{MHz}\right) \delta 34,21$.

HRMS (ESI) for $\mathrm{C}_{30} \mathrm{H}_{50} \mathrm{~B}_{2} \mathrm{NO}_{6} \mathrm{~S}\left[\mathrm{M}+\mathrm{H}^{+}\right]^{+}$: calculated: 574.3545 , found: 574.3543 .

N-(2-(bis(4,4,5,5-tetramethyl-1,3,2-dioxaborolan-2-yl)(trimethylsilyl)methyl)cyclohex-3-en-1yl)-4-methylbenzenesulfonamide (38)<smiles>C[Si](C)(C)C(Br)(Br)c1ccccc1</smiles>

Purified by flash column chromatography (hexane:ethyl acetate $=9: 1$ ) yielded 38 (99\%, $233 \mathrm{mg}$ ) as a white solid.

${ }^{1}{ }^{H}$ NMR $\left(\mathrm{CDCl}_{3}, 400 \mathrm{MHz}\right) \delta 7.75-7.67(\mathrm{~m}, 2 \mathrm{H}), 7.26-7.14(\mathrm{~m}, 2 \mathrm{H}), 5.51(\mathrm{br}, 2 \mathrm{H}), 5.28-5.15$ $(\mathrm{m}, 1 \mathrm{H}), 3.93(\mathrm{~m}, 1 \mathrm{H}), 2.42(\mathrm{~d}, \mathrm{~J}=4.1 \mathrm{~Hz}, 1 \mathrm{H}), 2.34(\mathrm{~s}, 3 \mathrm{H}), 1.99-1.77(\mathrm{~m}, 3 \mathrm{H}), 1.36(\mathrm{~m}, 1 \mathrm{H}), 1.14$ (m, 18H), $1.12(\mathrm{~s}, 6 \mathrm{H}), 0.00(\mathrm{~s}, 9 \mathrm{H})$.

${ }^{13} \mathrm{C} \mathrm{NMR}\left(\mathrm{CDCl}_{3}, 100 \mathrm{MHz}\right) \delta 141.83,139.03,129.31,128.69,126.17,126.13,82.10,81.85,52.26$, $40.34,24.35,24.20,24.13,24.01,23.45,20.77,20.72,0.00$.

${ }^{11} \mathrm{~B}$ NMR $\left(\mathrm{CDCl}_{3}, 128.3 \mathrm{MHz}\right) \delta 33.82$.

HRMS (ESI) for $\mathrm{C}_{29} \mathrm{H}_{50} \mathrm{~B}_{2} \mathrm{NO}_{6}$ SSi [M+H'] ${ }^{+}$: calculated: 590.3314 , found: 590.3321 . 
4-methyl-N-(2-((tetrahydro-2H-pyran-4-yl)bis(4,4,5,5-tetramethyl-1,3,2-dioxaborolan-2-

yl)methyl)cyclohex-3-en-1-yl)benzenesulfonamide (39)<smiles>[AlH2][C@H]1CCC=C[C@H]1C(Br)(Cc1ccccc1)C1CCOCC1</smiles>

Purified by flash column chromatography (hexane:ethyl acetate $=9: 1$ )

yielded 39 (96\%, $231 \mathrm{mg})$ as a white solid.

${ }^{1} \mathrm{H}$ NMR $\left(\mathrm{CDCl}_{3}, 400 \mathrm{MHz}\right) \delta 7.68(\mathrm{~d}, \mathrm{~J}=8.3 \mathrm{~Hz}, 2 \mathrm{H}), 7.20(\mathrm{~d}, \mathrm{~J}=6.9 \mathrm{~Hz}, 2 \mathrm{H}), 5.625 .54(\mathrm{~m}, 1 \mathrm{H})$, $5.53-5.42(m, 1 H), 5.36(d, J=7.6 H z, 1 H), 3.85-3.73(m, 1 H), 3.73-3.61(m, 1 H), 3.61-3.49$ $(\mathrm{m}, 1 \mathrm{H}), 3.18(\mathrm{td}, \mathrm{J}=11.3,2.3 \mathrm{~Hz}, 1 \mathrm{H}), 3.04(\mathrm{td}, \mathrm{J}=11.2,3.0 \mathrm{~Hz}, 1 \mathrm{H}), 2.56(\mathrm{~m}, 1 \mathrm{H}), 2.35(\mathrm{~s}, 3 \mathrm{H})$, $1.98-1.79(\mathrm{~m}, 3 \mathrm{H}), 1.75-1.57(\mathrm{~m}, 2 \mathrm{H}), 1.56-1.41(\mathrm{~m}, 2 \mathrm{H}), 1.39-1.25(\mathrm{~m}, 2 \mathrm{H}), 1.20(\mathrm{~s}, 12 \mathrm{H})$, $1.09(\mathrm{~s}, 12 \mathrm{H})$.

${ }^{13} \mathrm{C} \mathrm{NMR}\left(\mathrm{CDCl}_{3}, 100 \mathrm{MHz}\right) \delta 142.85,139.24,131.47,129.50,126.99,125.95,83.50,83.08,68.88$, $68.73,50.59,42.25,36.25,31.92,31.04,26.30,25.12,25.05,24.71,24.63,22.21,21.47$.

${ }^{11} \mathbf{B}$ NMR $\left(\mathrm{CDCl}_{3}, 128.3 \mathrm{MHz}\right) \delta 32,26$.

HRMS (ESI) for $\mathrm{C}_{31} \mathrm{H}_{50} \mathrm{~B}_{2} \mathrm{NO}_{7} \mathrm{~S}\left[\mathrm{M}+\mathrm{H}^{+}\right]^{+}$: calculated: 602.3494 , found: 602.3508 .

4-methyl-N-(2-(2-phenyl-1,1-bis(4,4,5,5-tetramethyl-1,3,2-dioxaborolan-2-yl)ethyl)cyclohex3-en-1-yl)benzenesulfonamide (40)<smiles></smiles>

Purified by flash column chromatography (hexane:ethyl acetate $=9: 1)$ yielded $\mathbf{4 0}(51 \%, 124 \mathrm{mg}$ ) as a white solid.

${ }^{1}{ }_{H}$ NMR $\left(\mathrm{CDCl}_{3}, 400 \mathrm{MHz}\right) \delta 7.79-7.71(\mathrm{~m}, 2 \mathrm{H}), 7.33-7.21(\mathrm{~m}, 4 \mathrm{H}), 7.14-7.04(\mathrm{~m}, 3 \mathrm{H}), 5.28$ (dq, J = 10.1, 2.1 Hz, 1H), $5.16(\mathrm{br}, 2 \mathrm{H}), 3.70(\mathrm{~m}, 1 \mathrm{H}), 3.08(\mathrm{~d}, \mathrm{~J}=14.6 \mathrm{~Hz}, 1 \mathrm{H}), 2.93(\mathrm{~d}, \mathrm{~J}=14.6 \mathrm{~Hz}$, 1H), $2.66(\mathrm{~m}, 1 \mathrm{H}), 2.38(\mathrm{~s}, 3 \mathrm{H}), 1.83-1.57(\mathrm{~m}, 3 \mathrm{H}), 1.35-1.34(\mathrm{~m}, 1 \mathrm{H}), 1.29(\mathrm{~s}, 12 \mathrm{H}), 1.26(\mathrm{~s}$, $6 \mathrm{H}), 1.23(\mathrm{~s}, 6 \mathrm{H})$.

${ }^{13} \mathrm{C}$ NMR $\left(\mathrm{CDCl}_{3}, 100 \mathrm{MHz}\right) \delta 142.88,142.41,139.59,131.34,130.21,129.53,127.37,127.04$, $125.41,125.26,83.95,83.57,52.30,43.68,34.08,28.42,25.37,25.24,25.01,24.64,23.70,21.51$. ${ }^{11} \mathrm{~B}$ NMR $\left(\mathrm{CDCl}_{3}, 128.3 \mathrm{MHz}\right) \delta 35.22$.

HRMS (ESI) for $\mathrm{C}_{33} \mathrm{H}_{47} \mathrm{~B}_{2} \mathrm{NO}_{6} \mathrm{SNa}$ [M+Na+] ${ }^{+}$: calculated: 630.3207 , found: 630.3219 . 
4-methyl-N-(2-((4,4,5,5-tetramethyl-1,3,2-dioxaborolan-2-yl)(p-tolyl)methyl)cyclohex-3-en-1yl)benzenesulfonamide (41)<smiles>[3H]N[C@@H]1CCC=CC1C(Cc1ccccc1)c1ccc(C)cc1</smiles>

Purified by flash column chromatography (hexane:ethyl acetate $=9: 1$ ) yielded 41 (66\%, $127 \mathrm{mg}$ ) as a white solid.

${ }^{1} \mathrm{H}$ NMR $\left(\mathrm{CDCl}_{3}, 400 \mathrm{MHz}\right) \delta 7.37(\mathrm{~d}, \mathrm{~J}=8.3 \mathrm{~Hz}, 2 \mathrm{H}), 7.07(\mathrm{~d}, \mathrm{~J}=7.9 \mathrm{~Hz}, 2 \mathrm{H}), 6.87(\mathrm{~d}, \mathrm{~J}=7.7 \mathrm{~Hz}$, $2 \mathrm{H}), 6.77(\mathrm{~d}, \mathrm{~J}=8.1 \mathrm{~Hz}, 2 \mathrm{H}), 5.66(\mathrm{br}, 2 \mathrm{H}), 4.58(\mathrm{~d}, \mathrm{~J}=8.2 \mathrm{~Hz}, 1 \mathrm{H}), 3.11-3.03(\mathrm{~m}, 1 \mathrm{H}), 2.35(\mathrm{~s}$, $3 \mathrm{H}), 2.34-2.27(\mathrm{~m}, 1 \mathrm{H}), 2.24(\mathrm{~s}, 3 \mathrm{H}), 2.12-1.86(\mathrm{~m}, 3 \mathrm{H}), 1.70-1.58(\mathrm{~m}, 1 \mathrm{H}), 1.57-1.43(\mathrm{~m}$, $1 \mathrm{H}), 1.13-0.97(\mathrm{~m}, 12 \mathrm{H})$

${ }^{13} \mathrm{C}$ NMR $\left(\mathrm{CDCl}_{3}, 100 \mathrm{MHz}\right) \delta 142.60,137.92,136.35,134.95,129.38,129.27,128.57,128.41$, $127.55,126.97,83.52,48.78,43.13,24.53,22.26,21.55,21.07,20.52$.

${ }^{11}$ B NMR $\left(\mathrm{CDCl}_{3}, 128.3 \mathrm{MHz}\right) \delta 31.18$

HRMS (ESI) for $\mathrm{C}_{27} \mathrm{H}_{36} \mathrm{BNO}_{4} \mathrm{SNa}\left[\mathrm{M}+\mathrm{Na}^{+}\right]^{+}$: calculated: 504.2355 , found: 504.2358 . 
$\underline{{ }^{1} \mathrm{H},{ }^{13} \mathrm{C} \text { Spectra of vinyl aziridines }}$
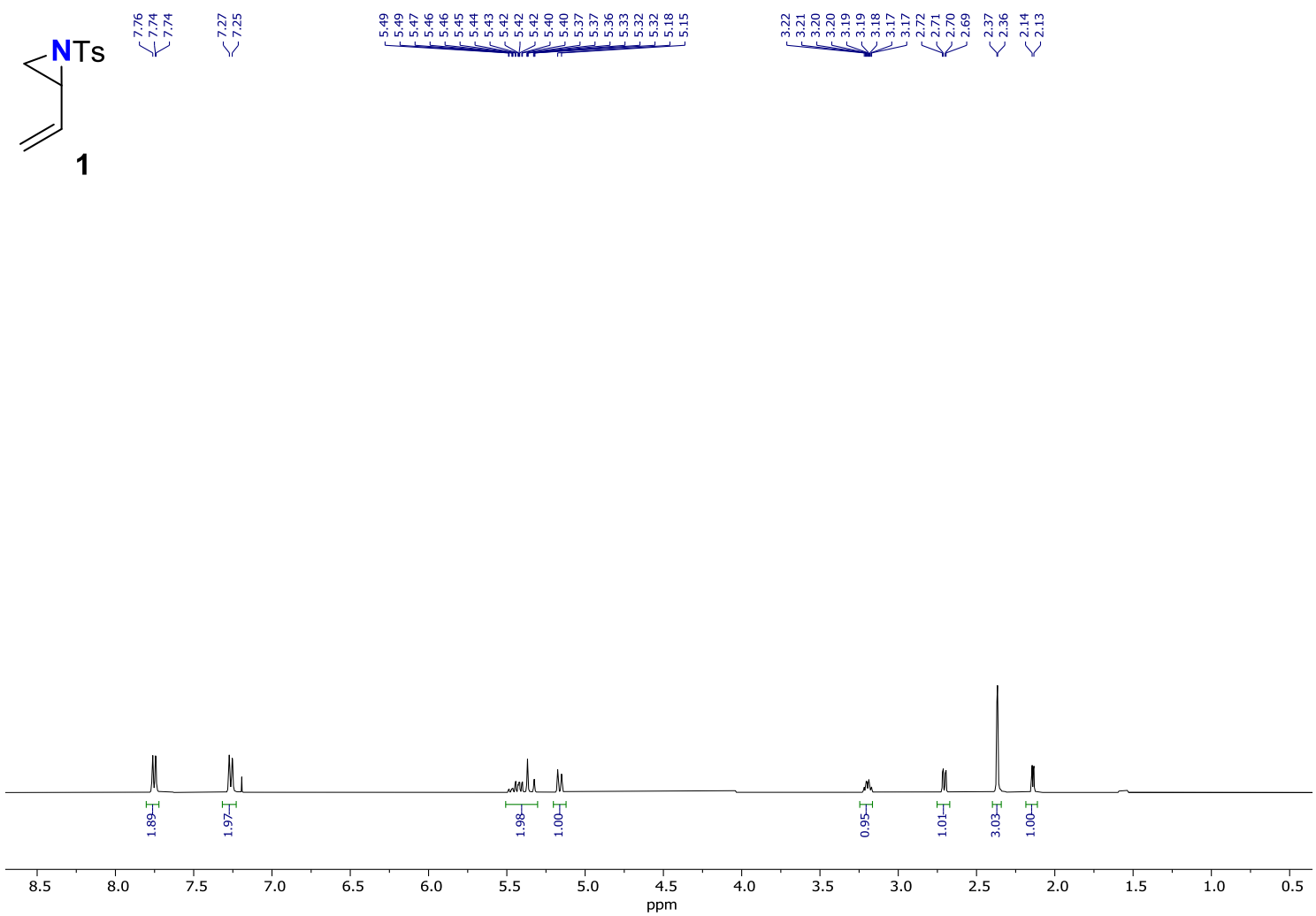

\begin{tabular}{|c|c|c|c|c|}
\hline 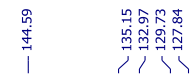 & 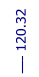 & 纱 & 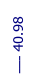 & $\stackrel{\substack{+\dot{m} \\
\mid}}{\mid}$ \\
\hline
\end{tabular}

$\int_{1}^{N T s}$

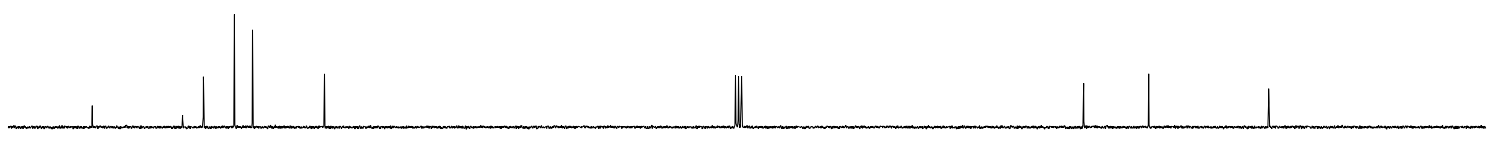

$\begin{array}{llllllllllllllllllllllllllllllllllllllll}150 & 145 & 140 & 135 & 130 & 125 & 120 & 115 & 110 & 105 & 100 & 95 & 90 & 85 & 80 & 75 & 70 & 65 & 60 & 55 & 50 & 45 & 40 & 35 & 30 & 25 & 20 & 15 & 10 & 5 & 0\end{array}$ 


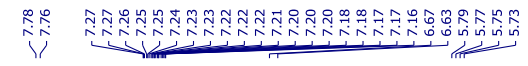

$\sum_{P h}^{N T s} 14$

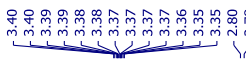

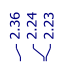

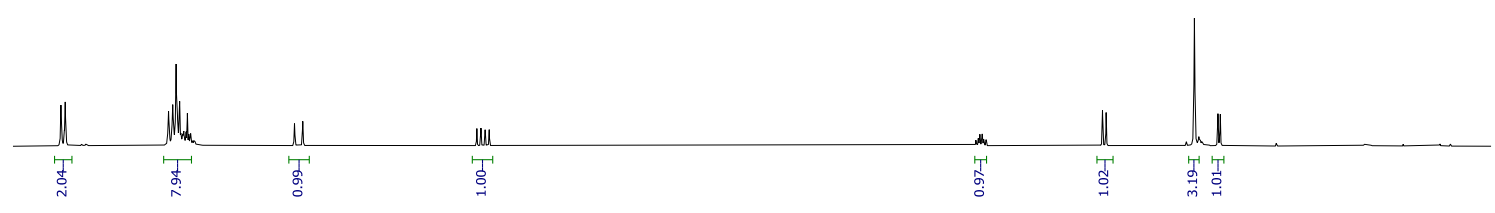

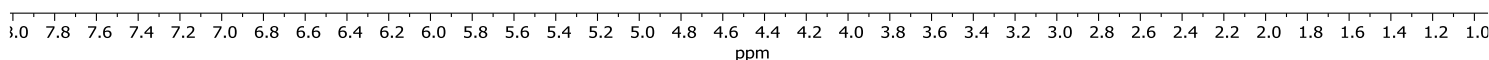<smiles>P/C=C\C1C[N+]1=[As]</smiles>

欲
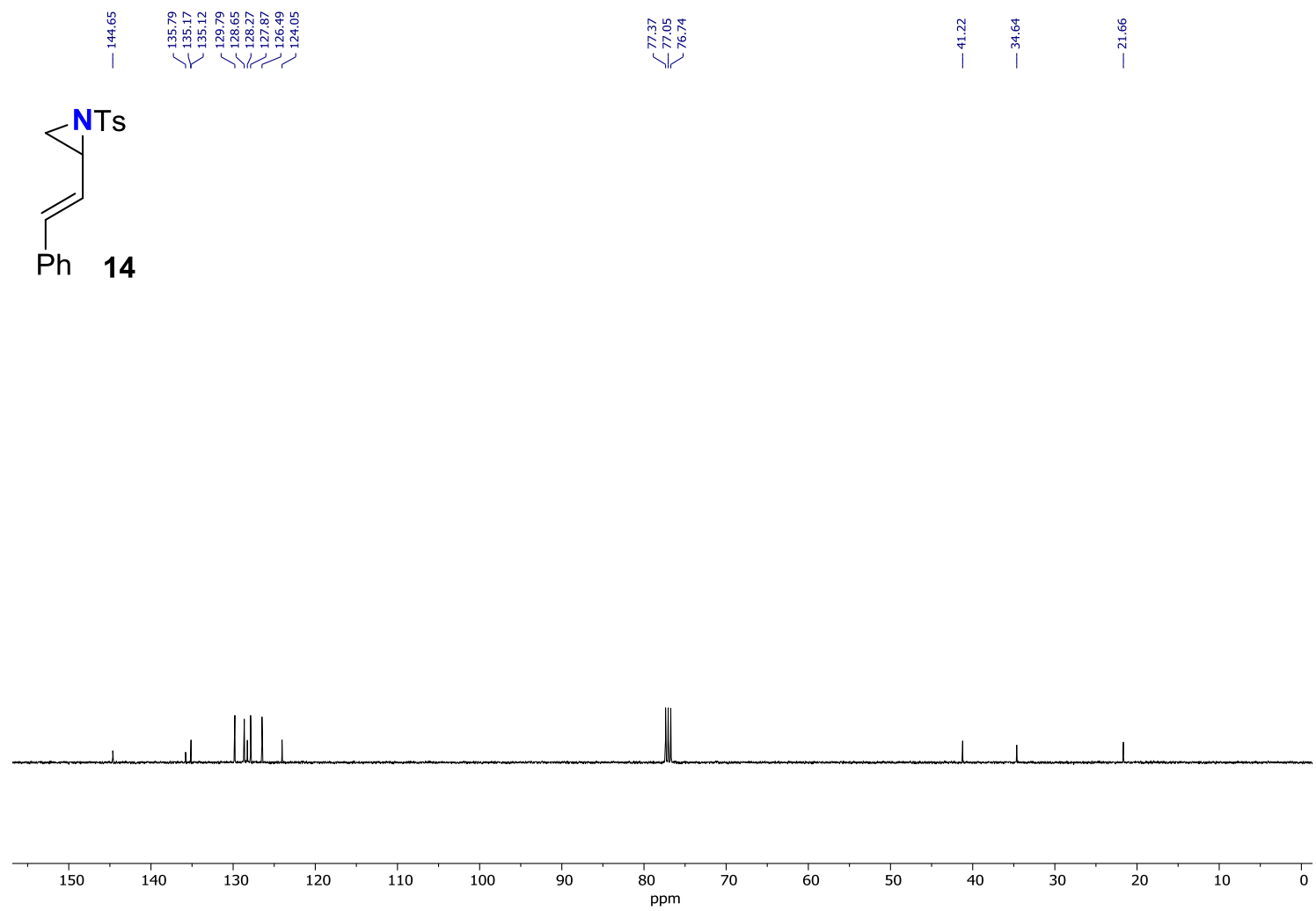

26 


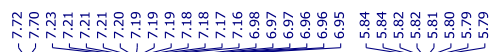

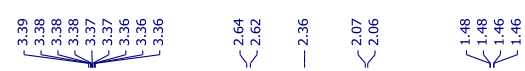<smiles>C/C=C(\c1ccccc1)C1CC1</smiles>

17

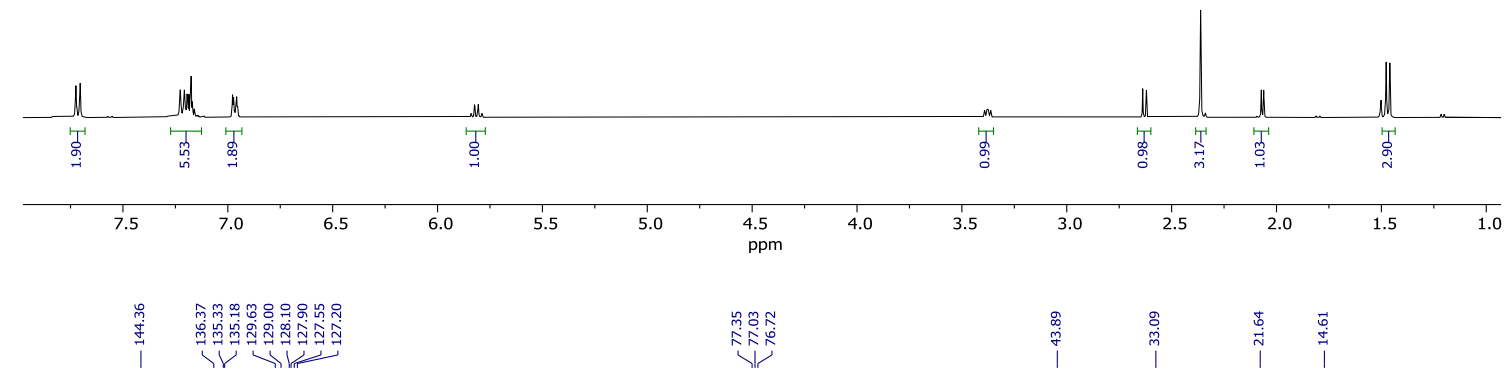

$\overbrace{P h}^{N T s}$

17

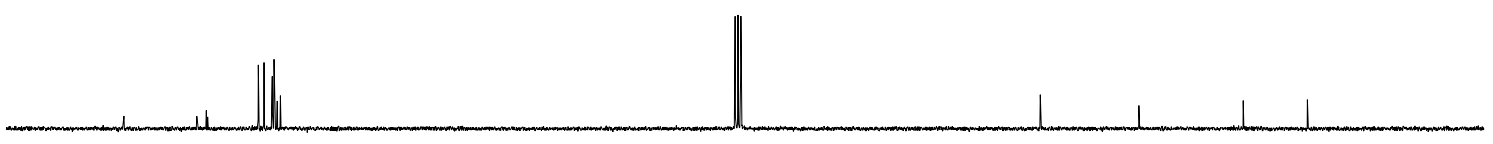

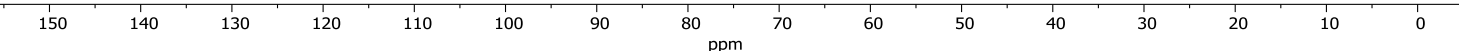




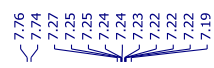

$\underbrace{N T s}_{20}+\underbrace{N T s}$

(ratio 2/1)

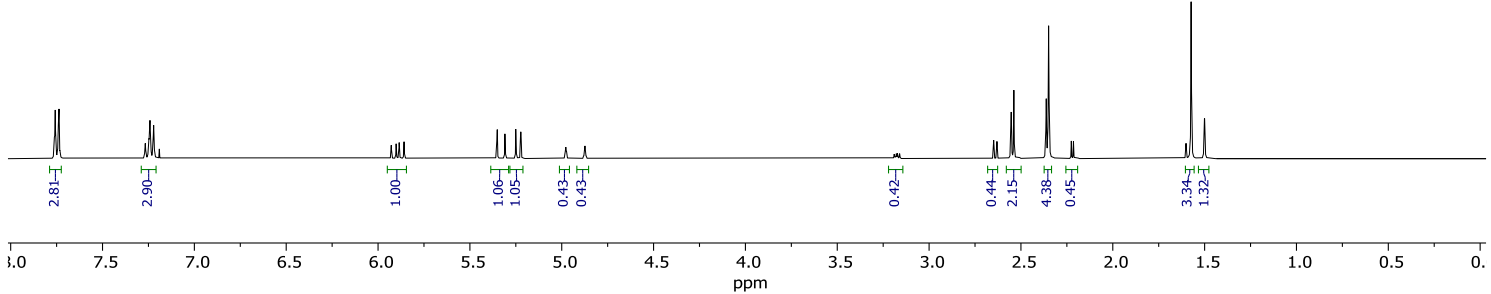

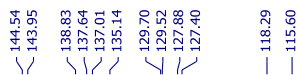

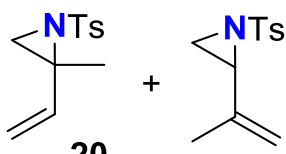

20

(ratio 2/1)

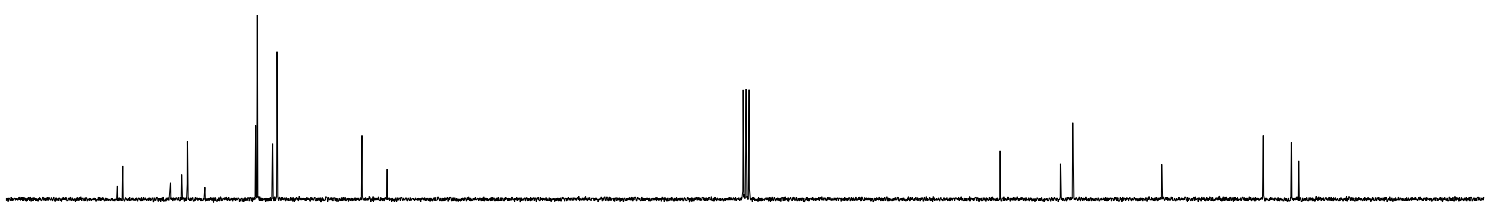


<smiles>[NH3+]C1C2C=CCCC12</smiles>

35
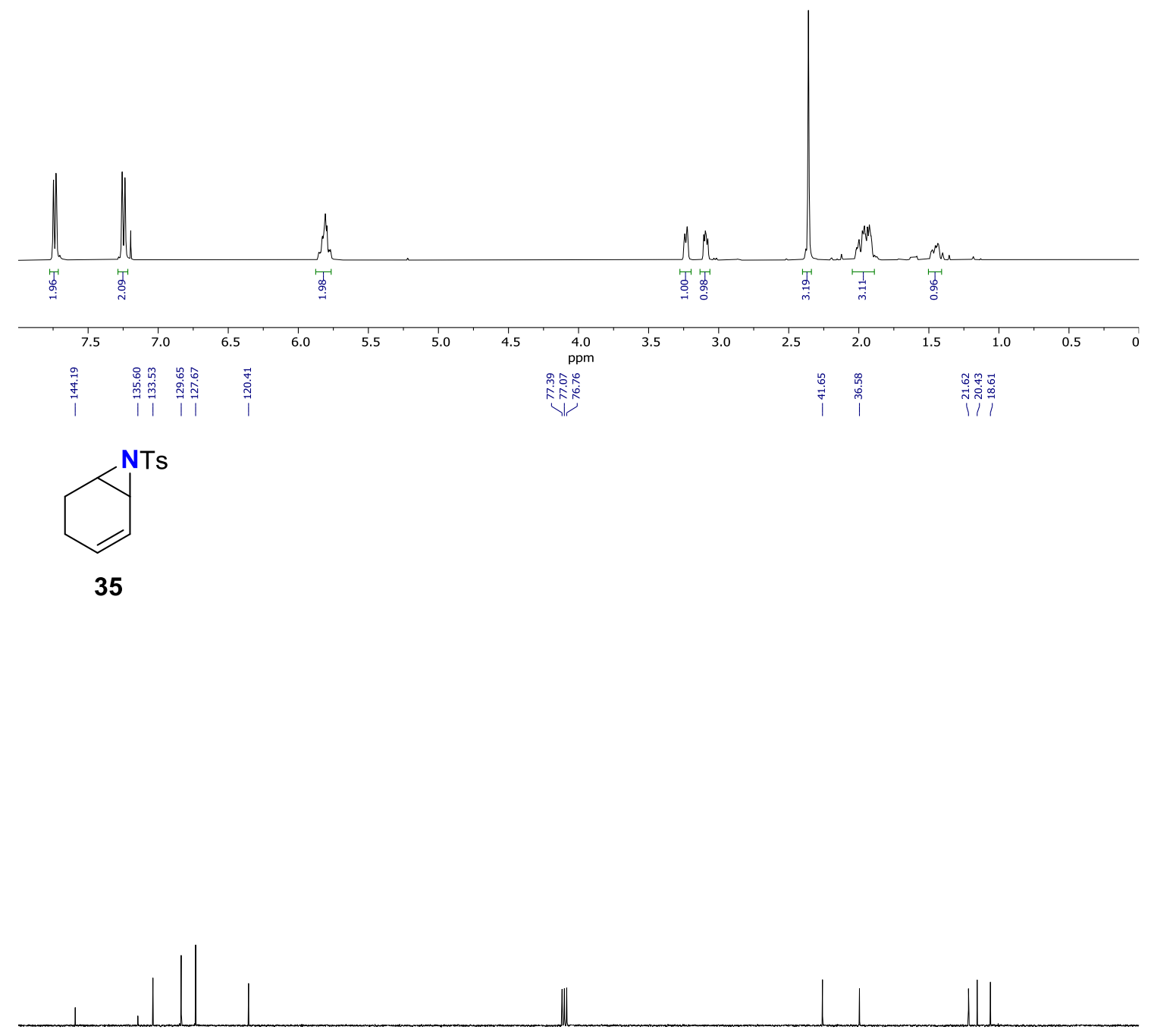

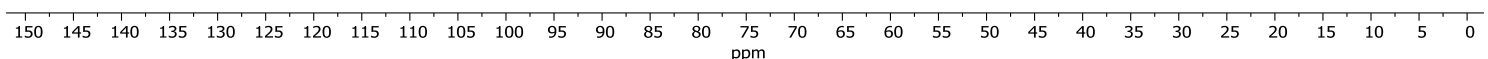


${ }^{1} \mathrm{H},{ }^{13} \mathrm{C},{ }^{11} \mathrm{~B}$ Spectra of geminal-diborylalkanes

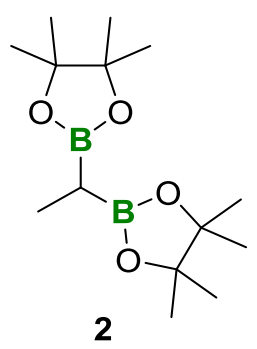

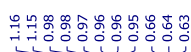

O.,

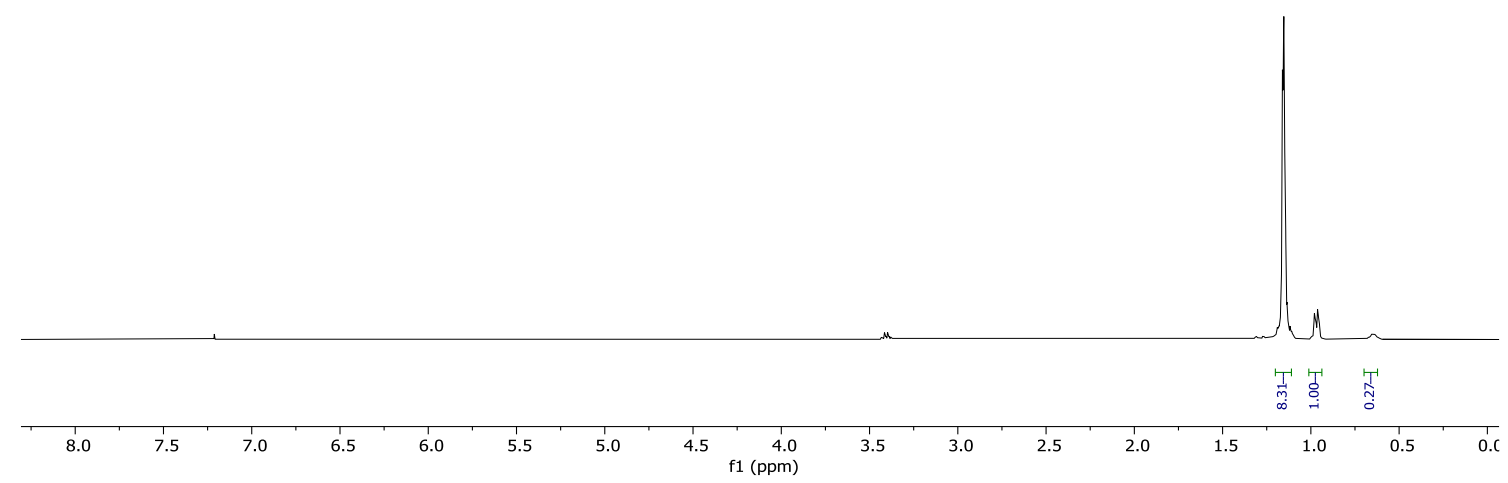<smiles>CC(B1OC(C)(C)C(C)(C)O1)B1OC(C)(C)C(C)(C)O1</smiles>

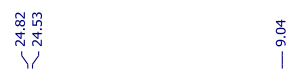

O., 0

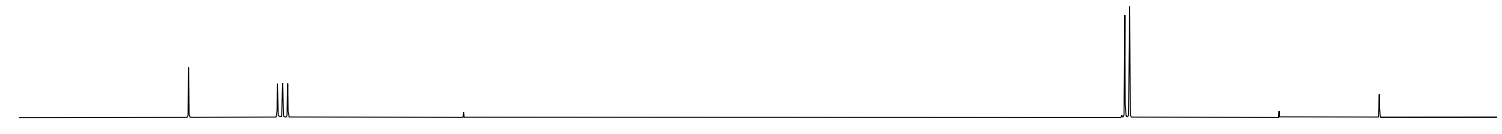

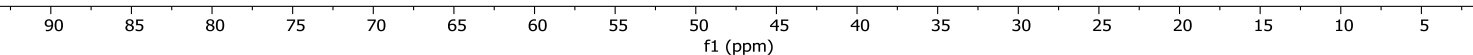



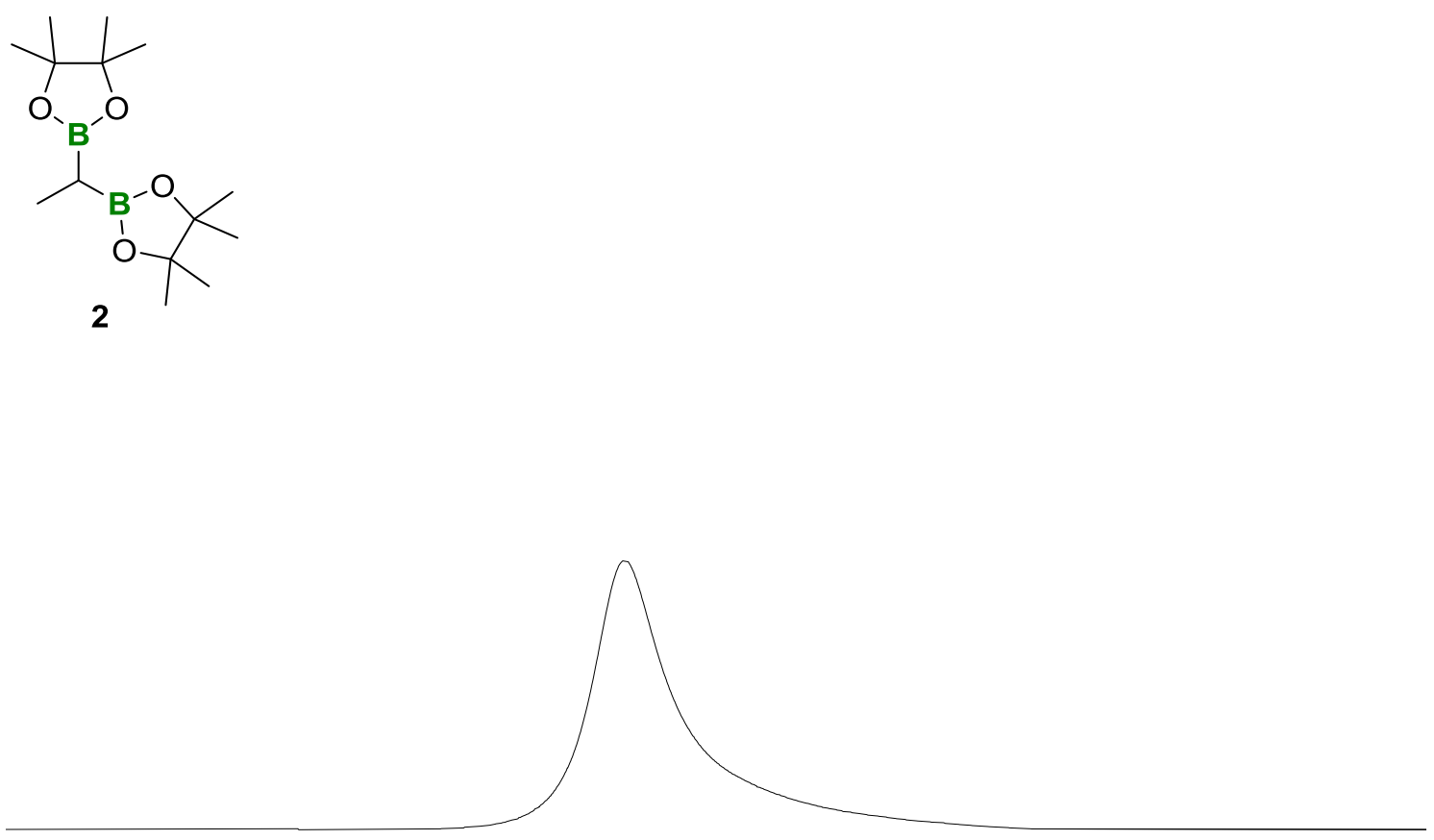

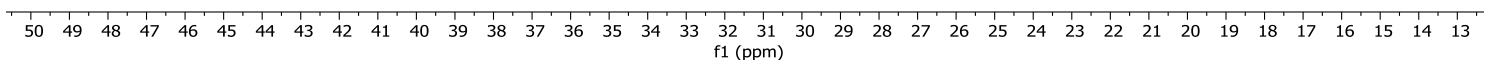

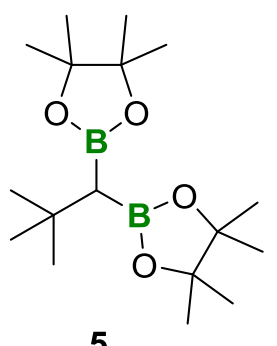

5

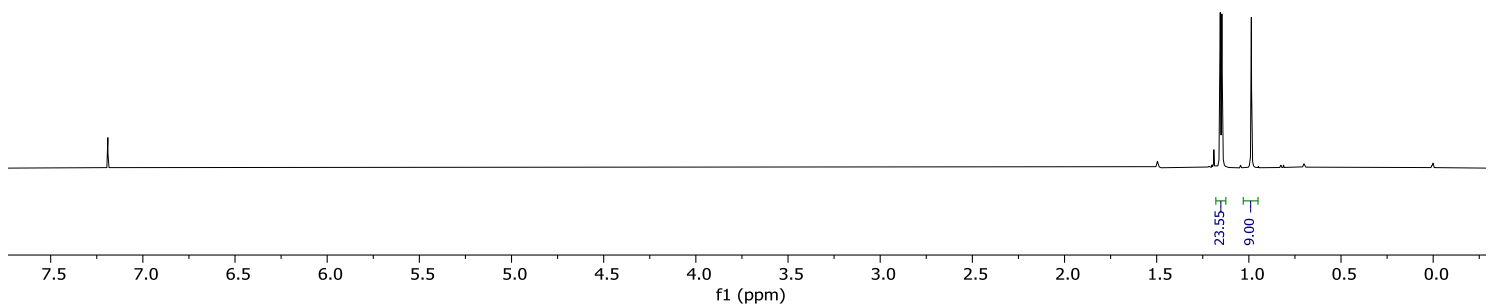




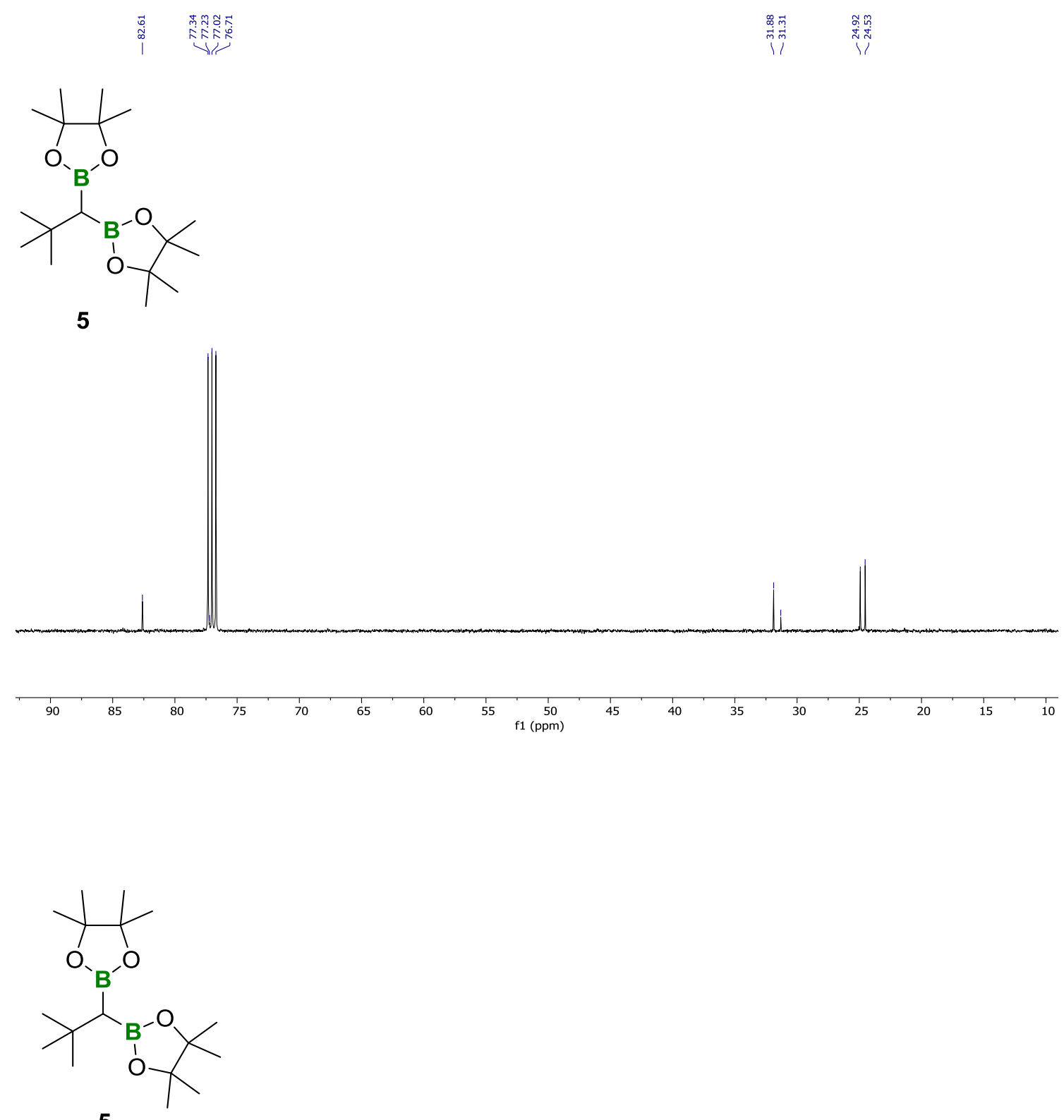

5

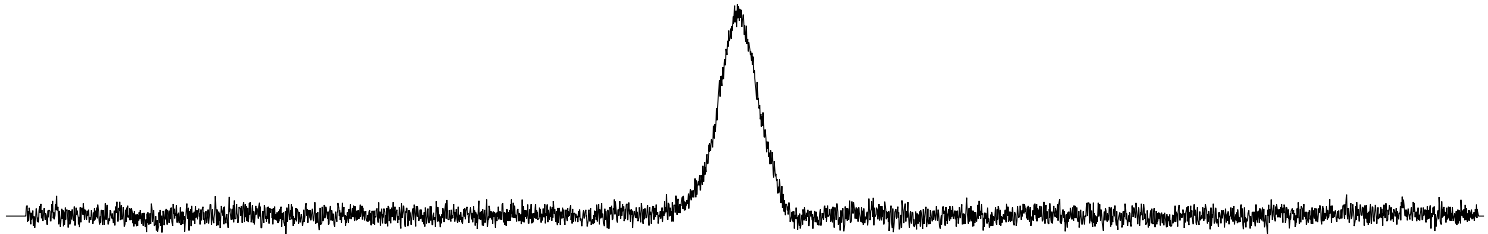

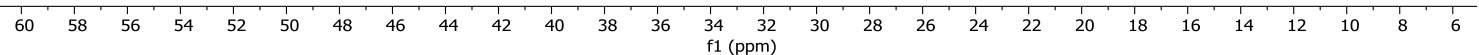



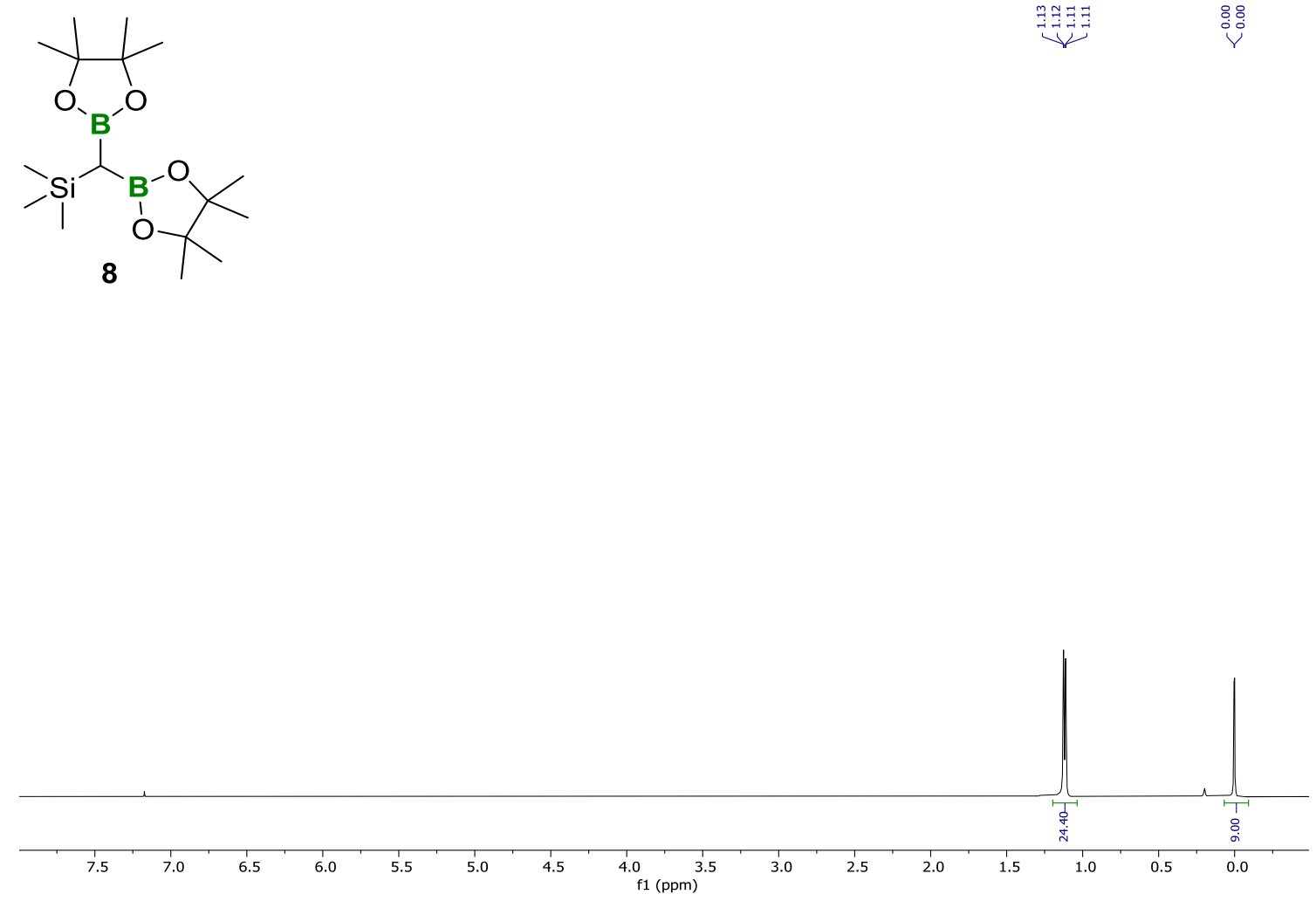<smiles>CC(C)(C)OB(OC(C)(C)C)OC(C)(C)C</smiles>

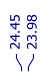

$\stackrel{\text { ò }}{i}$

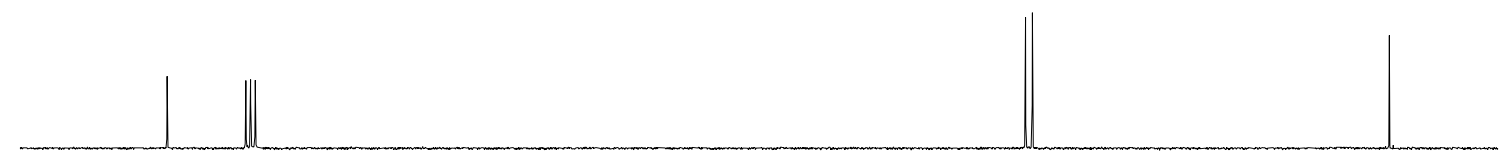

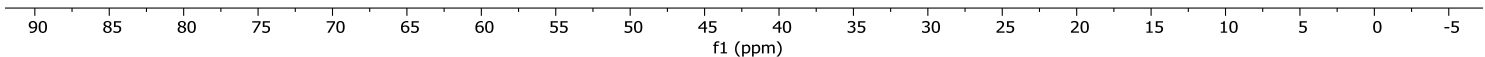



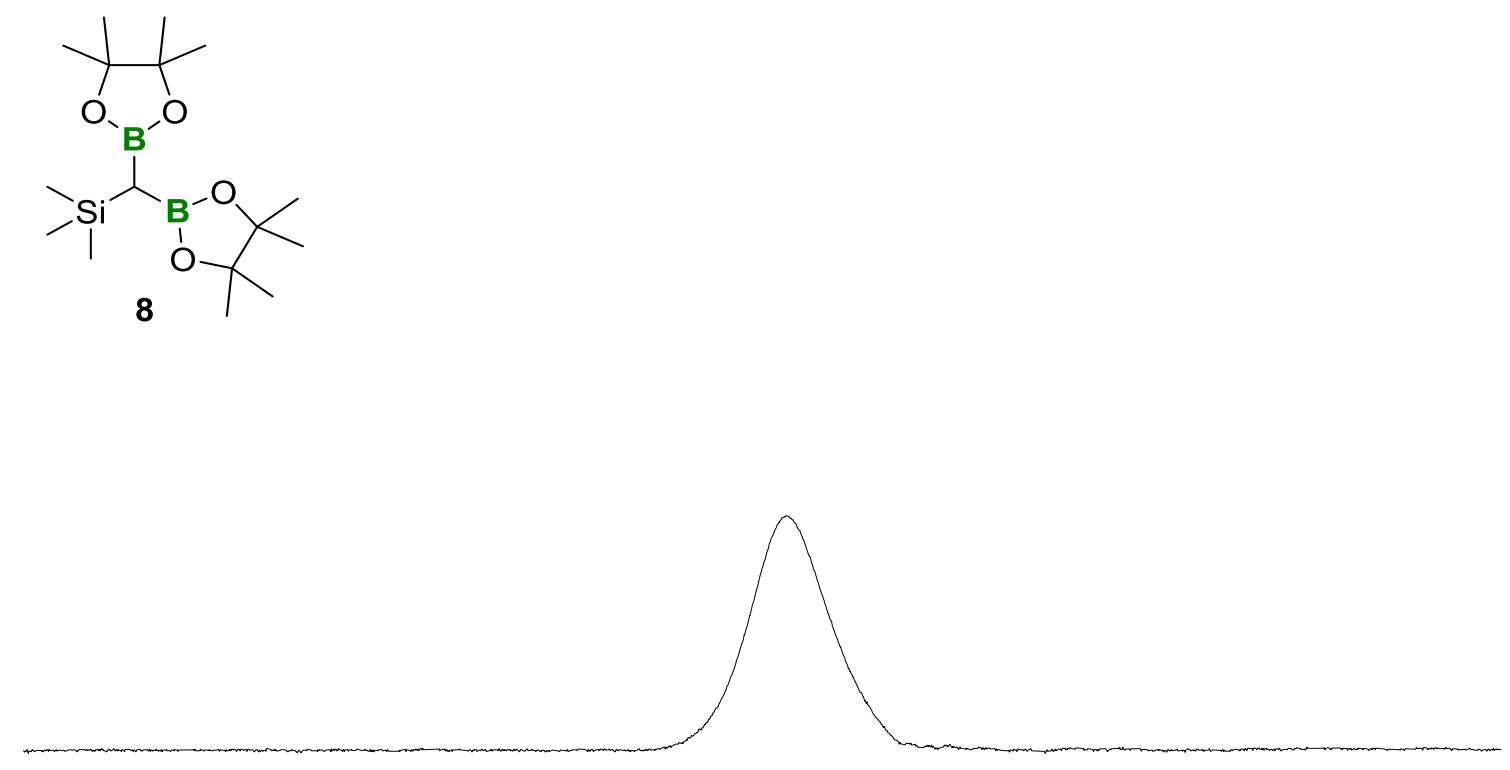

$\begin{array}{lllllllllllllllllllllllllllllllllllllll}49 & 48 & 47 & 46 & 45 & 44 & 43 & 42 & 41 & 40 & 39 & 38 & 37 & 36 & 35 & 34 & 33 & 32 & 31 & 30 & 29 & 28 & 27 & 26 & 25 & 24 & 23 & 22 & 21 & 20 & 19 & 18\end{array}$

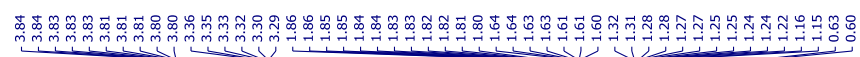

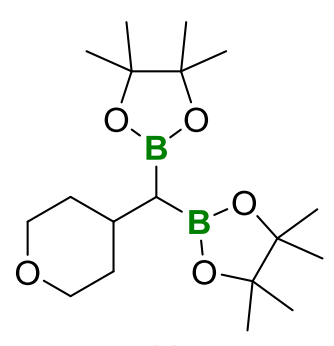

11

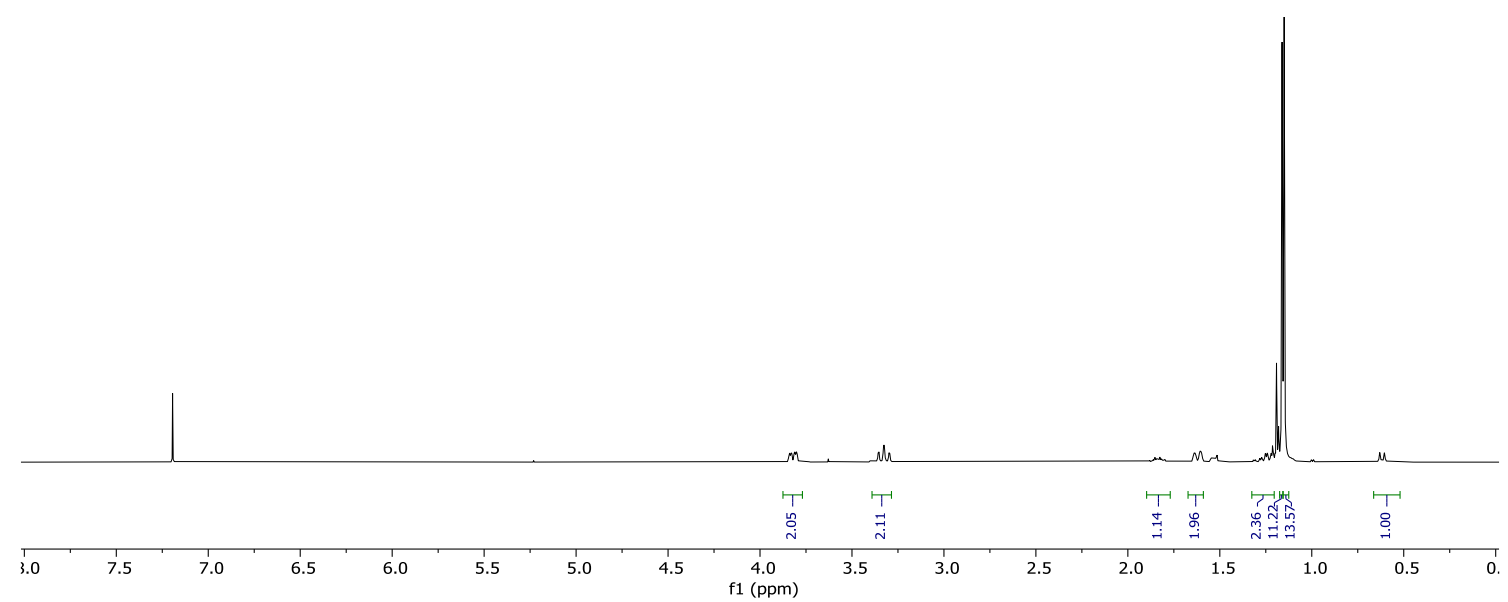



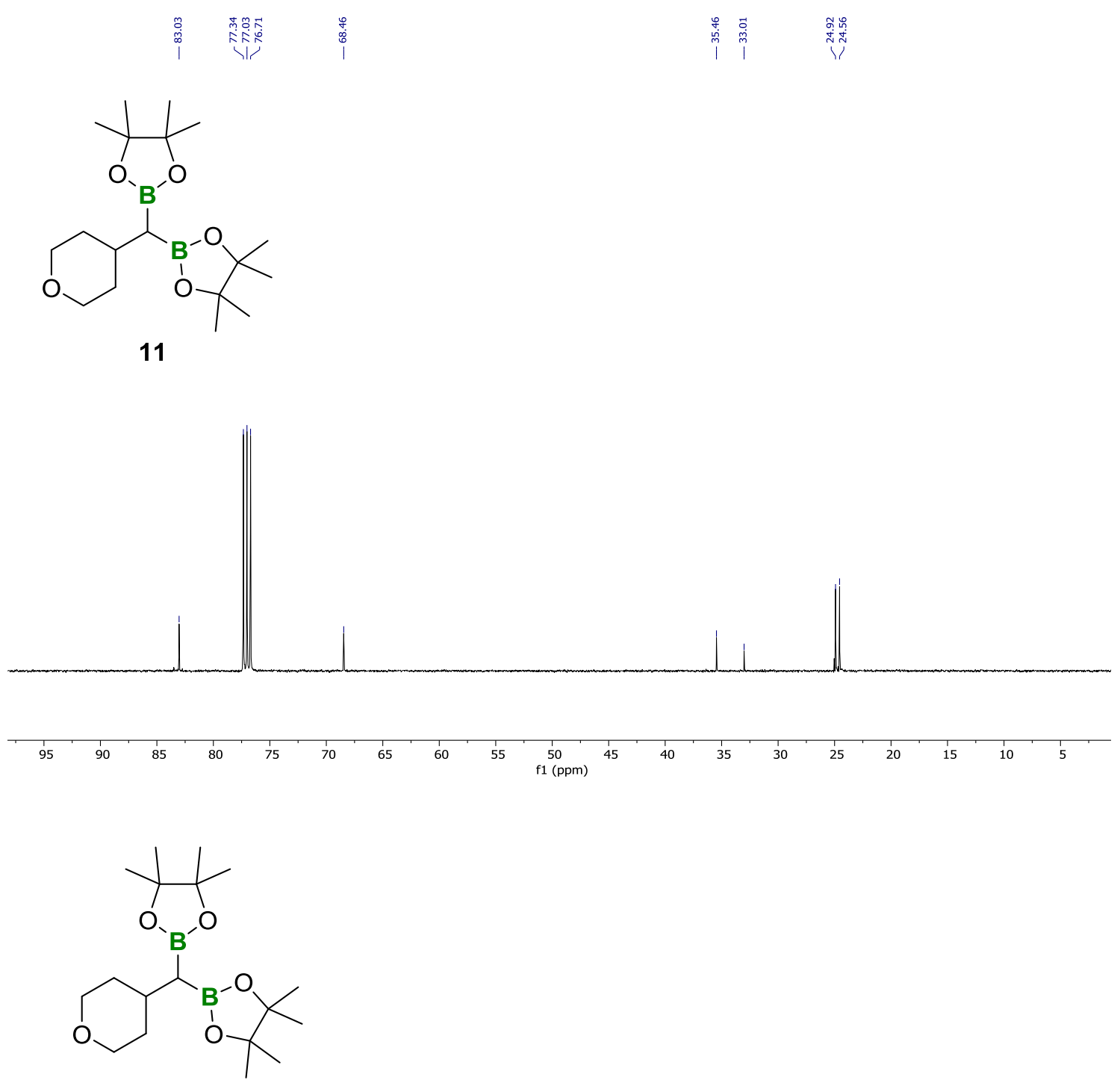

11

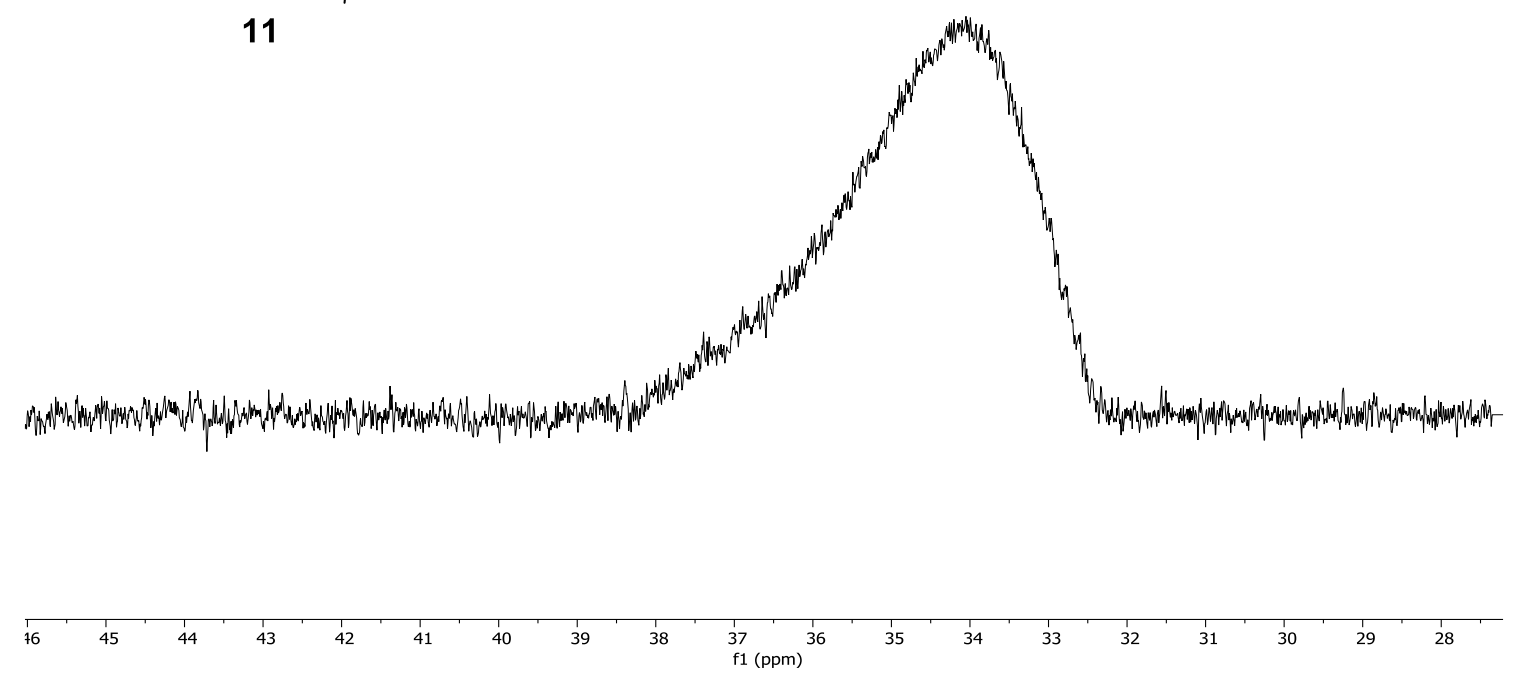




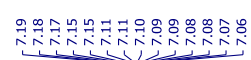<smiles>CC1(C)OB(C(CCc2ccccc2)B2OC(C)(C)C(C)(C)O2)OC1(C)C</smiles>
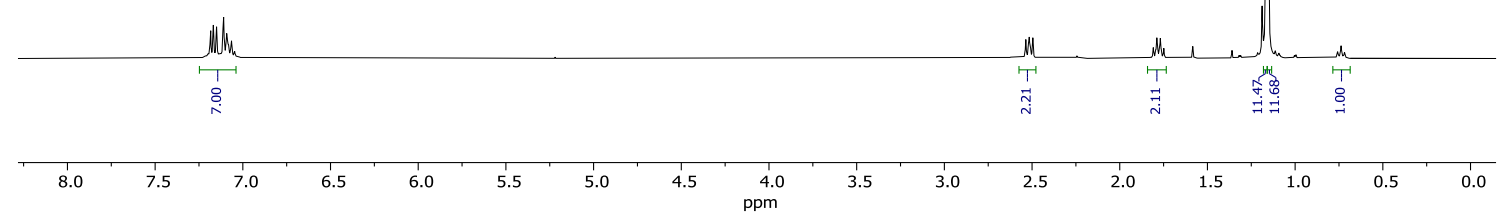

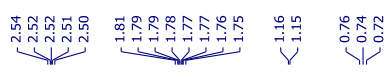

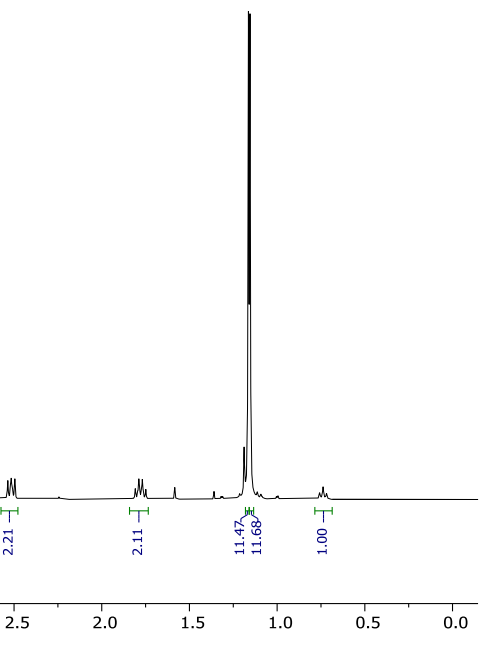

总

1<smiles>CC1(C)OB(C(CCc2ccccc2)B2OC(C)(C)C(C)(C)O2)OC1(C)C</smiles>

25

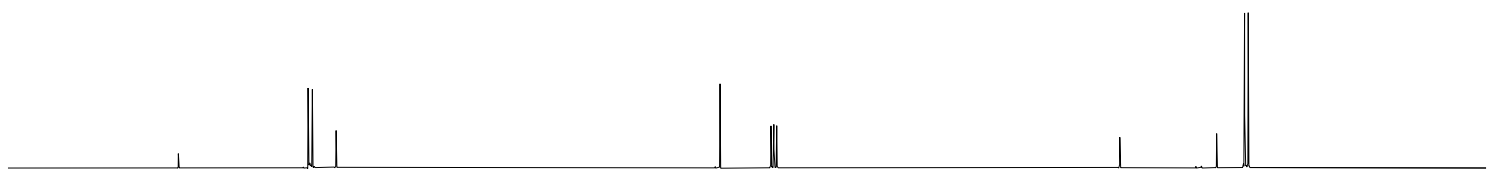

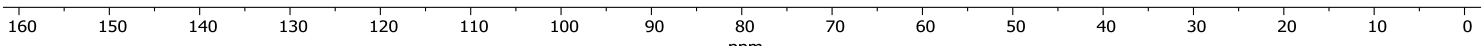



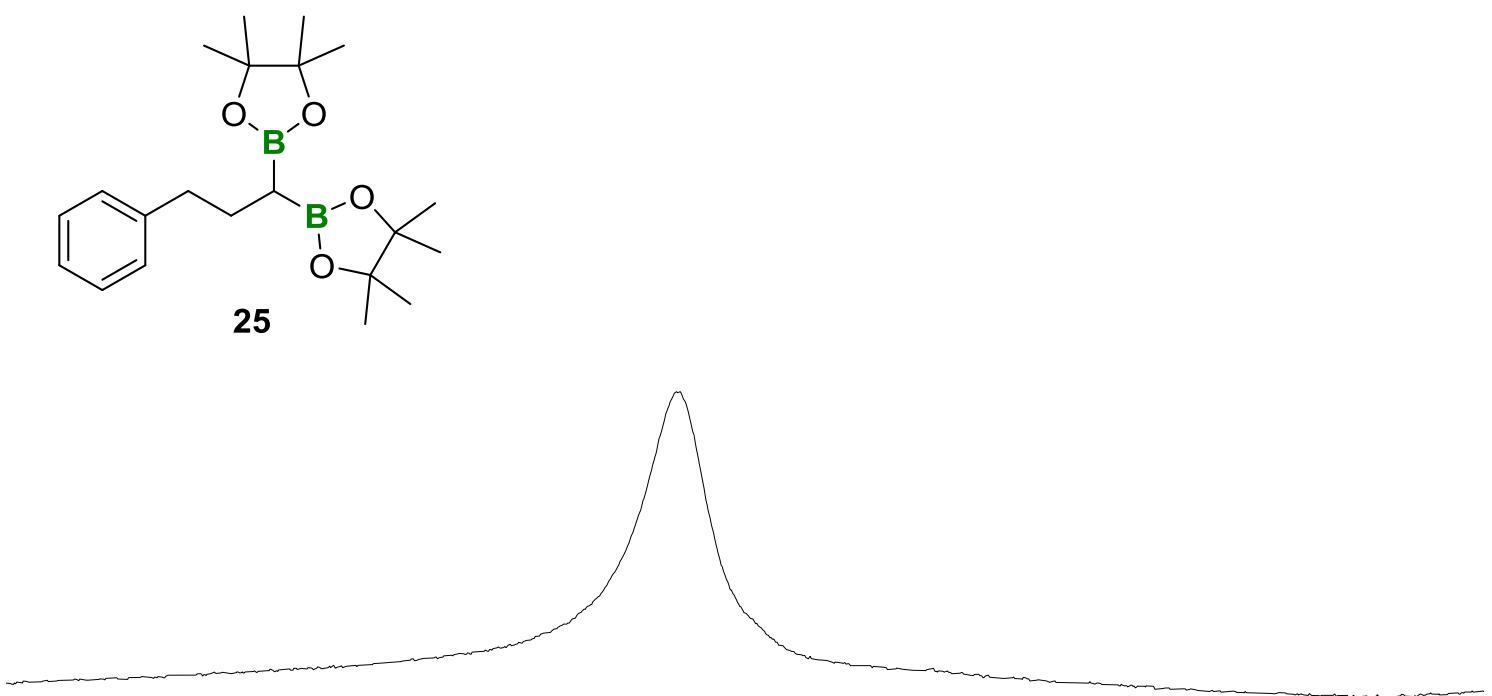

\begin{tabular}{llllllllllllllllllllllllllllllllllllllllllll}
\hline 64 & 62 & 60 & 58 & 56 & 54 & 52 & 50 & 48 & 46 & 44 & 42 & 40 & 38 & 36 & 34 & 32 & 30 & 28 & 26 & 24 & 22 & 20 & 18 & 16 & 14 & 12 & 10 & 8 & 6 & 4 & 1 & 0 & -2
\end{tabular}

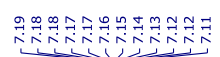

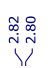

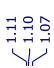<smiles>[Z7]CC(B1OC(C)(C)C(C)(C)O1)B1OC(C)(C)C(C)(C)O1</smiles>

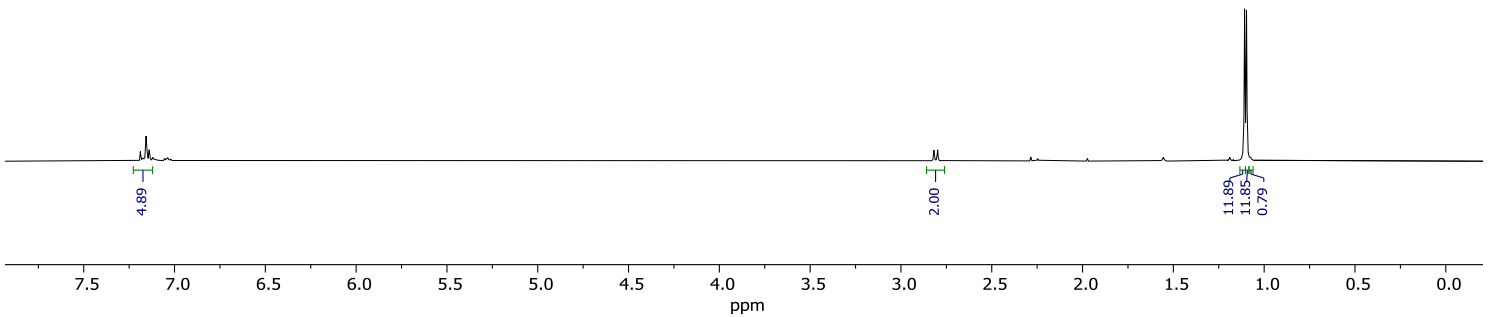



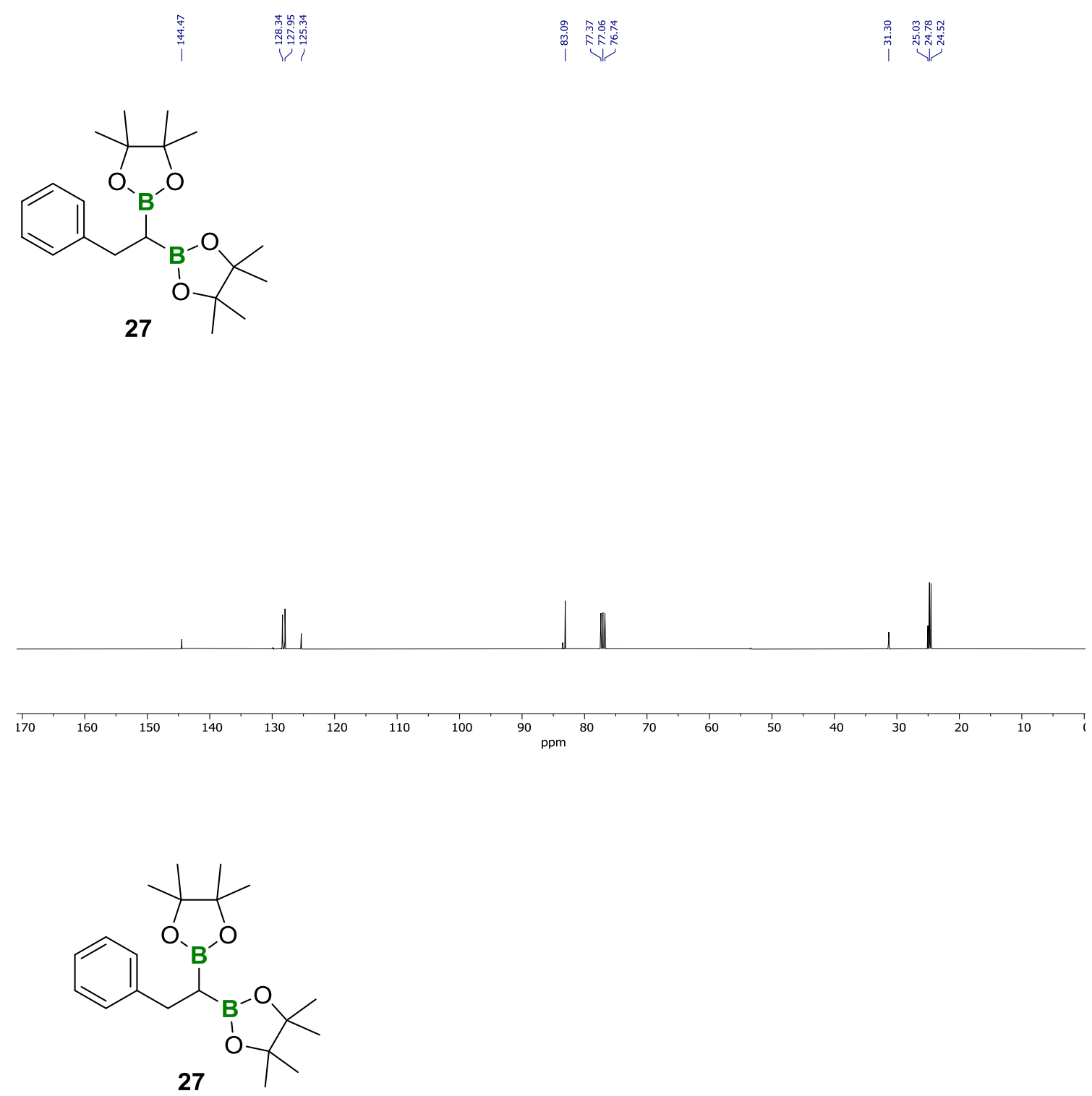

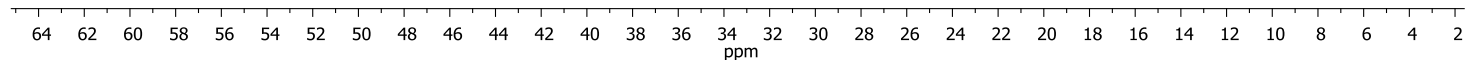




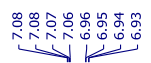
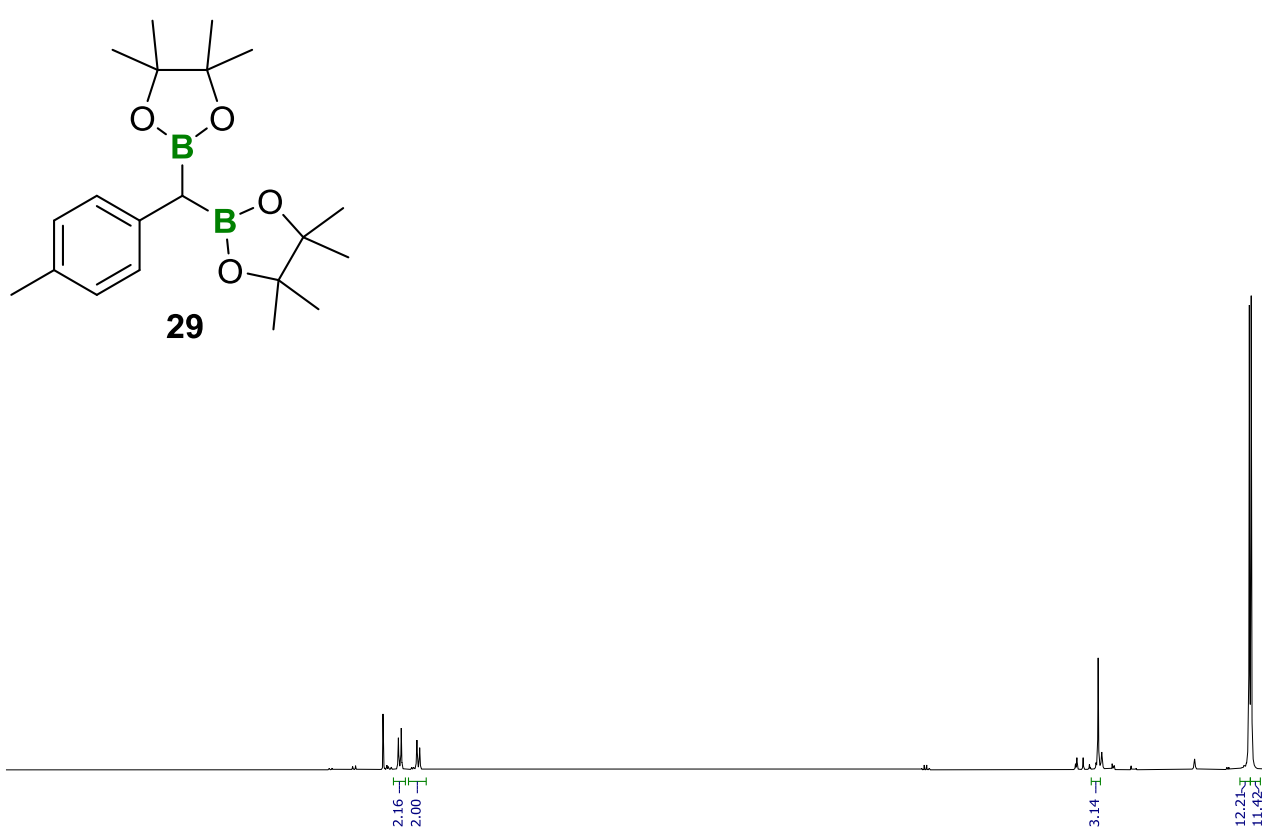

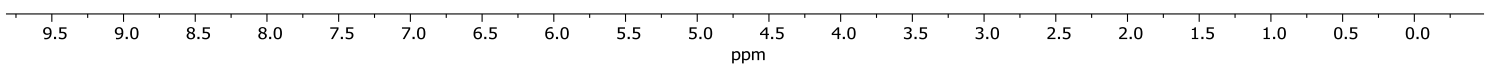

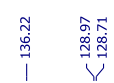

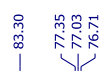

ป
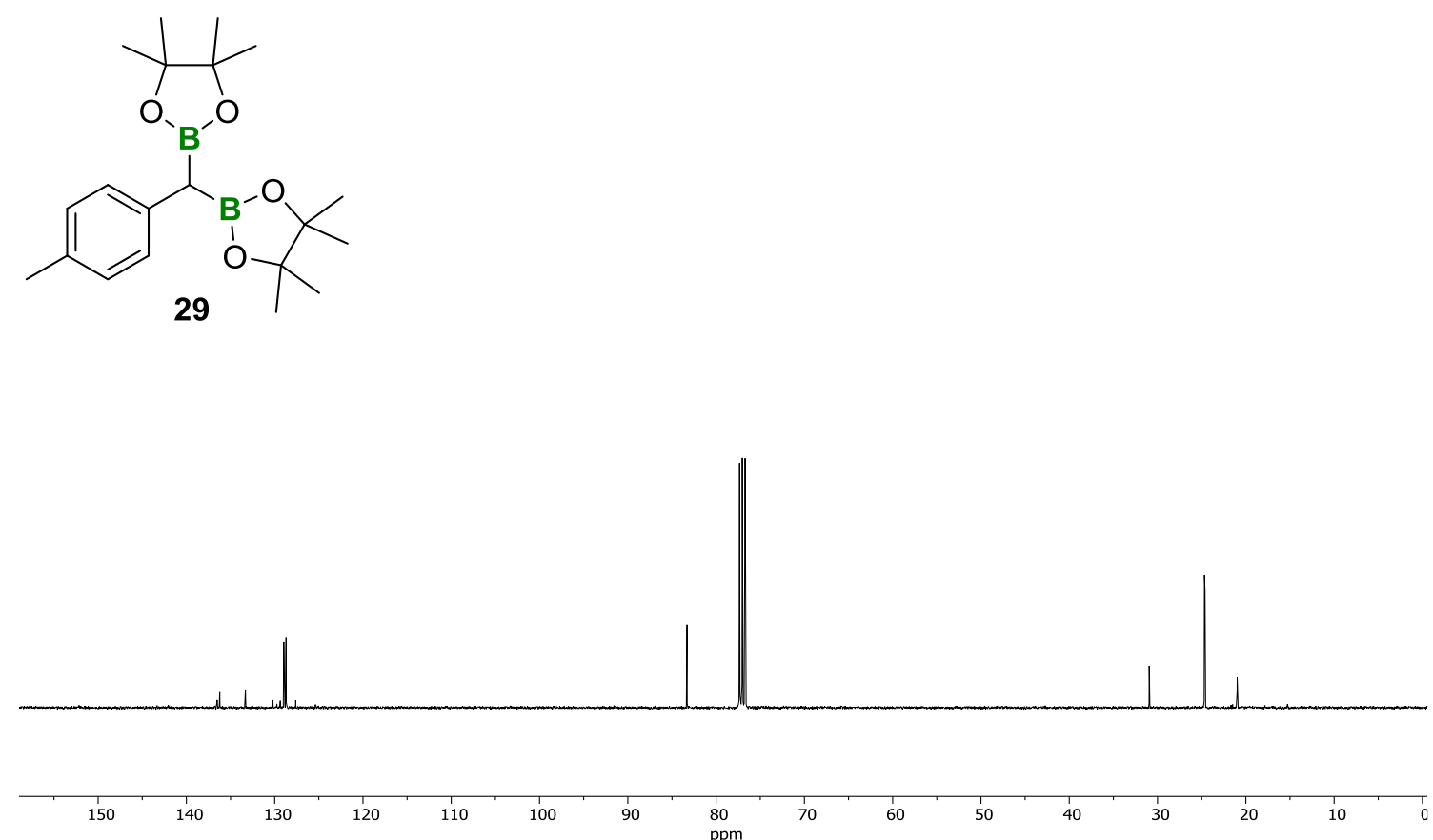

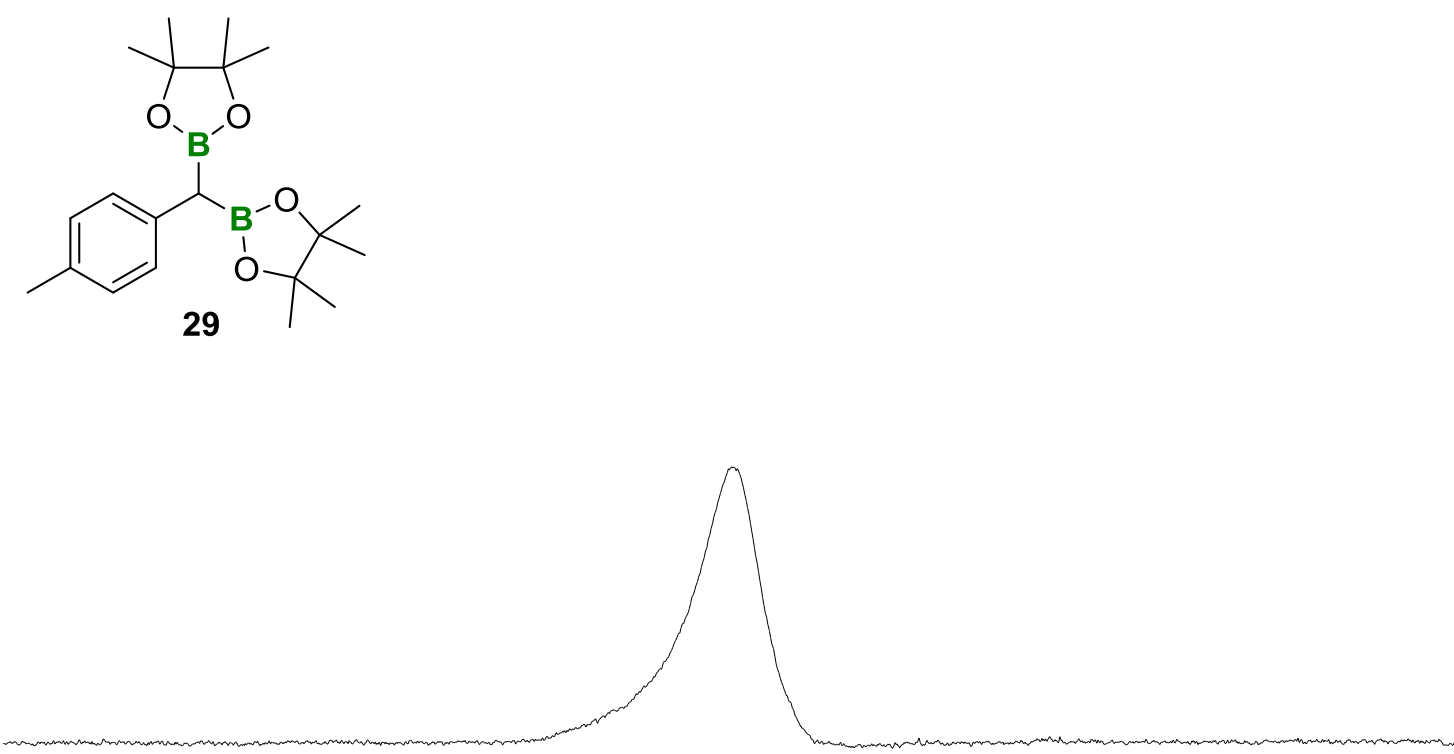

$\begin{array}{lllllllllllllllllllllllllllllll}56 & 54 & 52 & 50 & 48 & 46 & 44 & 42 & 40 & 38 & 36 & 34 & 32 & 30 & 28 & 26 & 24 & 22 & 20 & 18 & 16 & 14 & 12 & 10\end{array}$ 
$\underline{{ }^{1} \mathrm{H},{ }^{13} \mathrm{C},{ }^{11} \mathrm{~B} \text { Spectra of organoborane products }}$

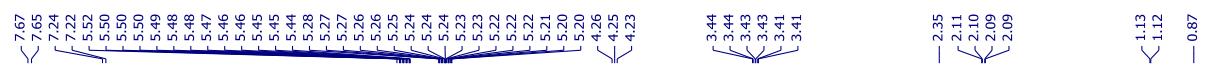

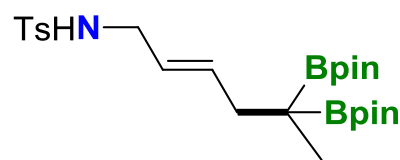

3
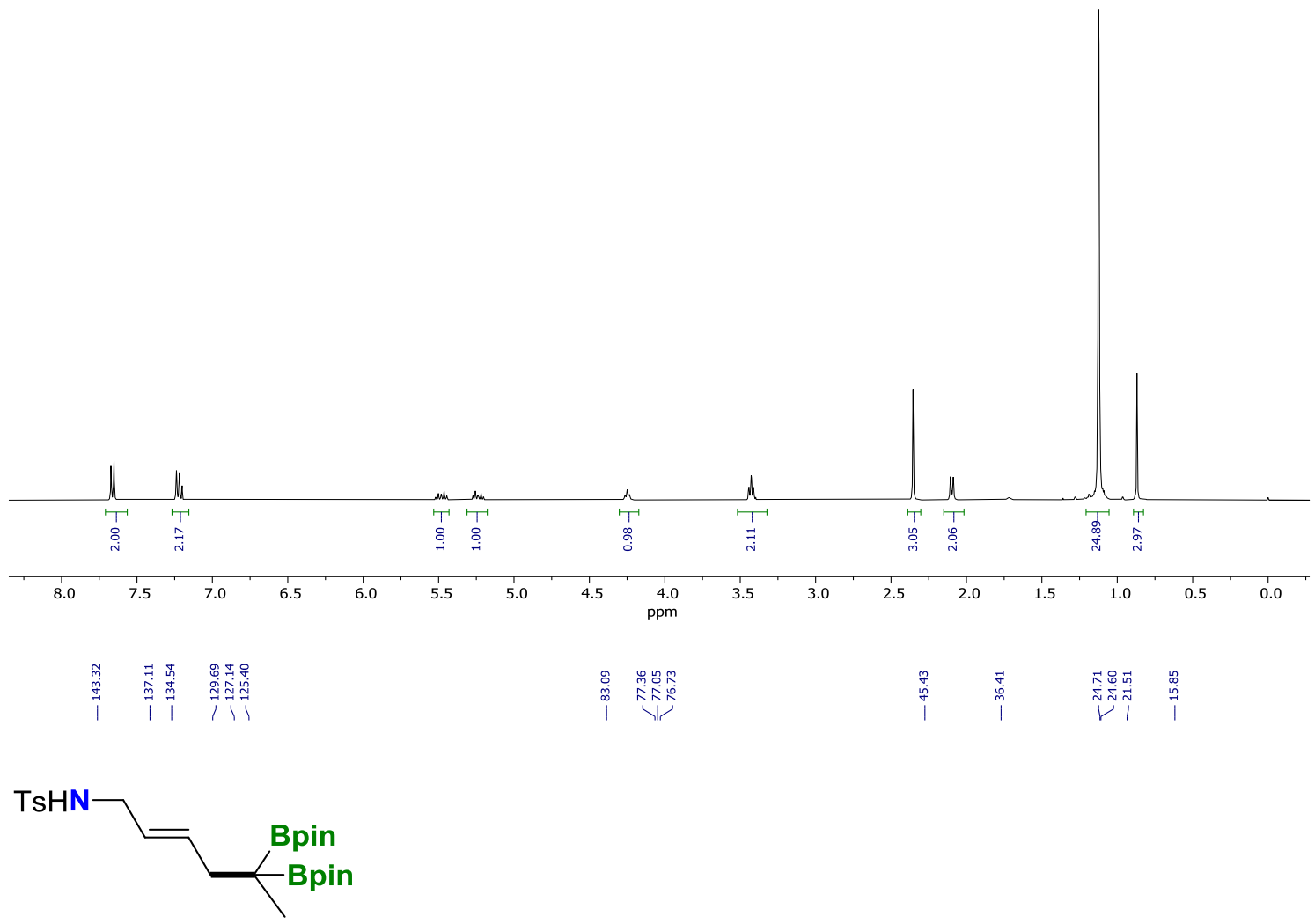

3

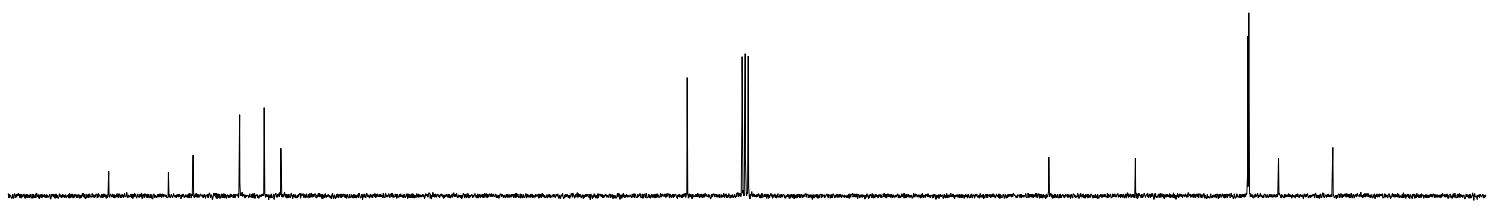

$\begin{array}{llllllllllllllllllllllllllllllllllllllllllllll}150 & 145 & 140 & 135 & 130 & 125 & 120 & 115 & 110 & 105 & 100 & 95 & 90 & 85 & 80 & 75 & 70 & 65 & 60 & 55 & 50 & 45 & 40 & 35 & 30 & 25 & 20 & 15 & 10 & 5 & 1\end{array}$ 


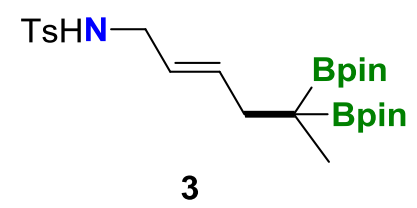

3

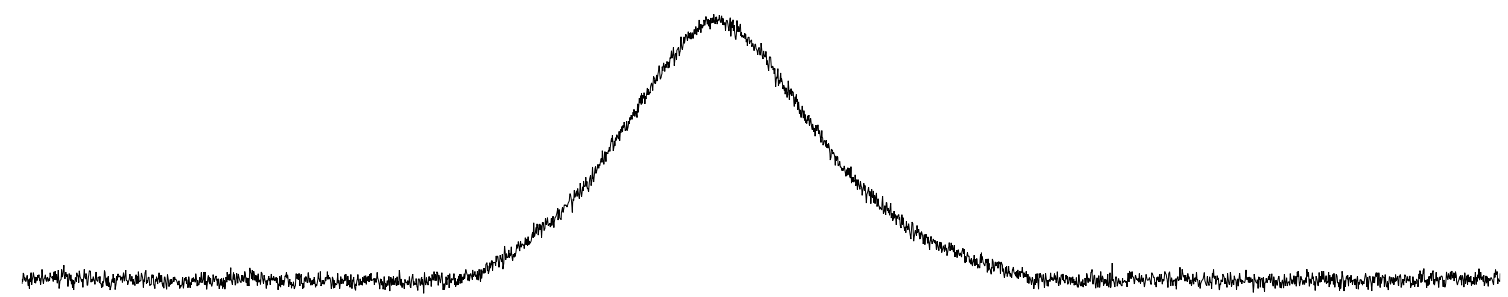

\begin{tabular}{llllllllllllllllllllllllllllllllllllllllllllllllllll}
\hline 49 & 48 & 47 & 46 & 45 & 44 & 43 & 42 & 41 & 40 & 39 & 38 & 37 & 36 & 35 & 34 & 33 & 32 & 31 & 30 & 29 & 28 & 27 & 26 & 25 & 24 & 23 & 22 & 21 & 20 & 19 & 18 & 1
\end{tabular}

戠

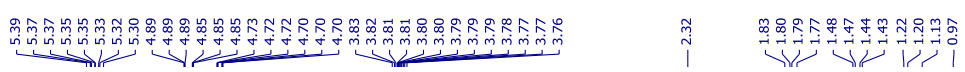

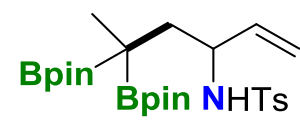

4

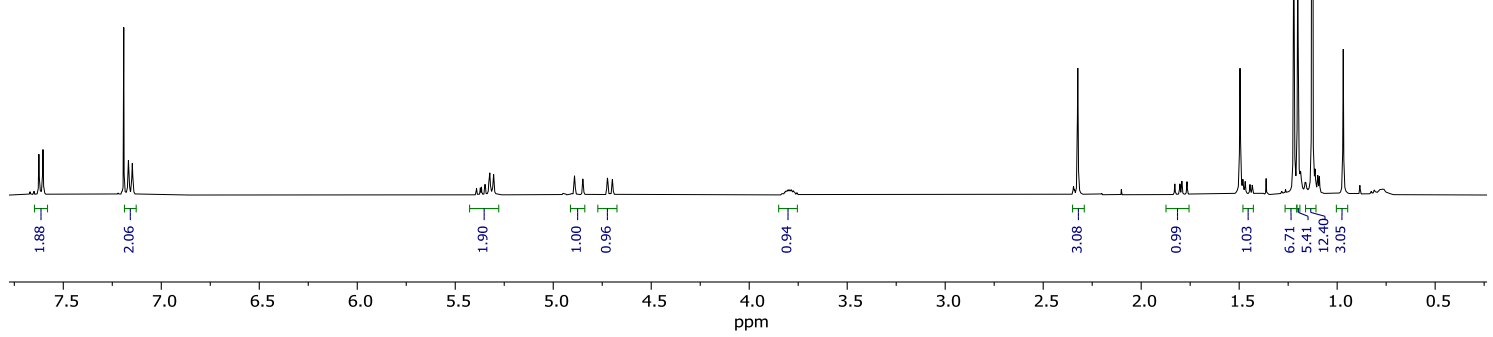



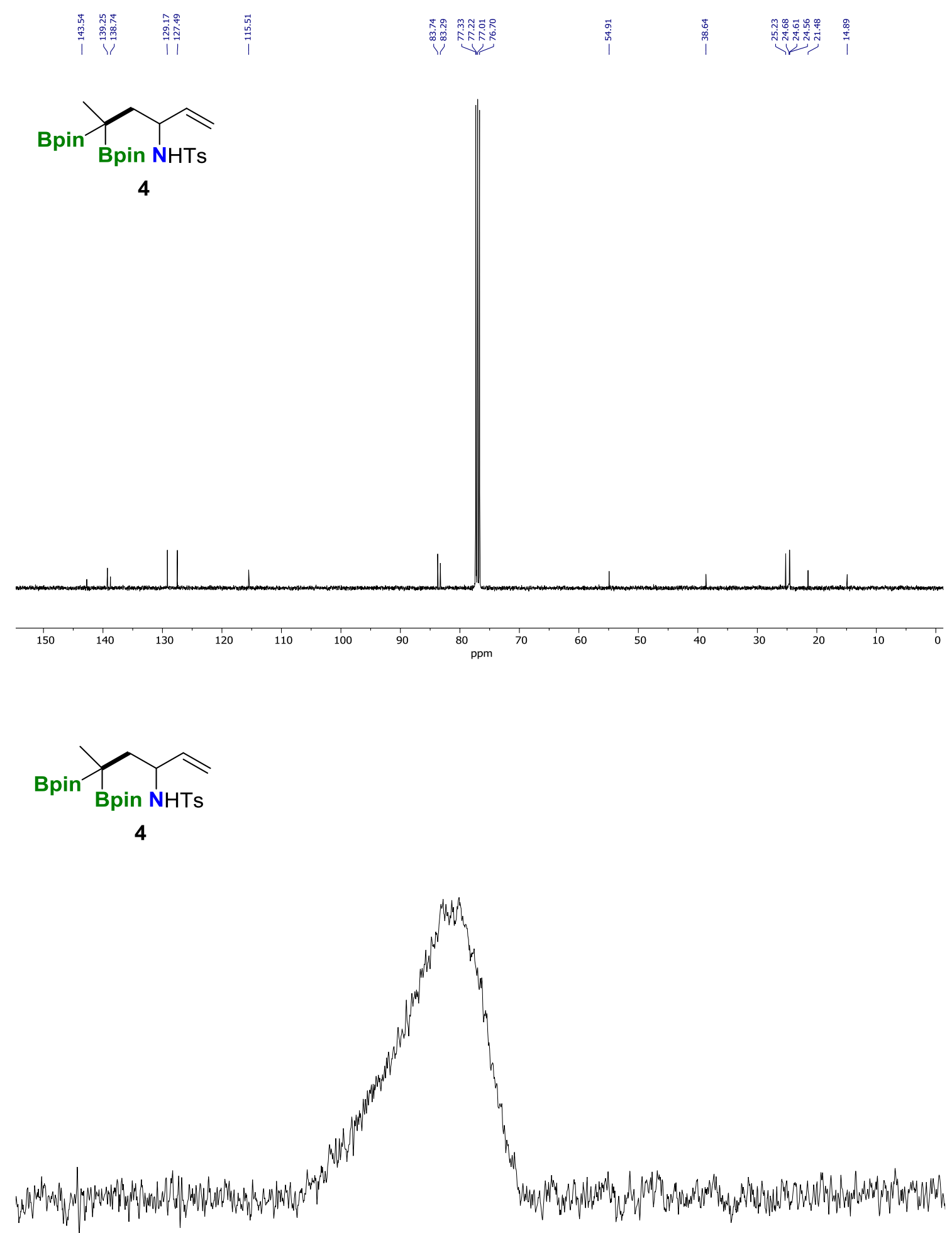

$\begin{array}{lllllllllllllllllllllllllllllllllllllllllllllllll}54 & 53 & 52 & 51 & 50 & 49 & 48 & 47 & 46 & 45 & 44 & 43 & 42 & 41 & 40 & 39 & 38 & 37 & 36 & 35 & 34 & 33 & 32 & 31 & 30 & 29 & 28 & 27 & 26 & 25 & 24 & 23 & 22 & 21 & 20 & 19 & 18 & 17 & 16 & 15 & 14 & 13\end{array}$ 

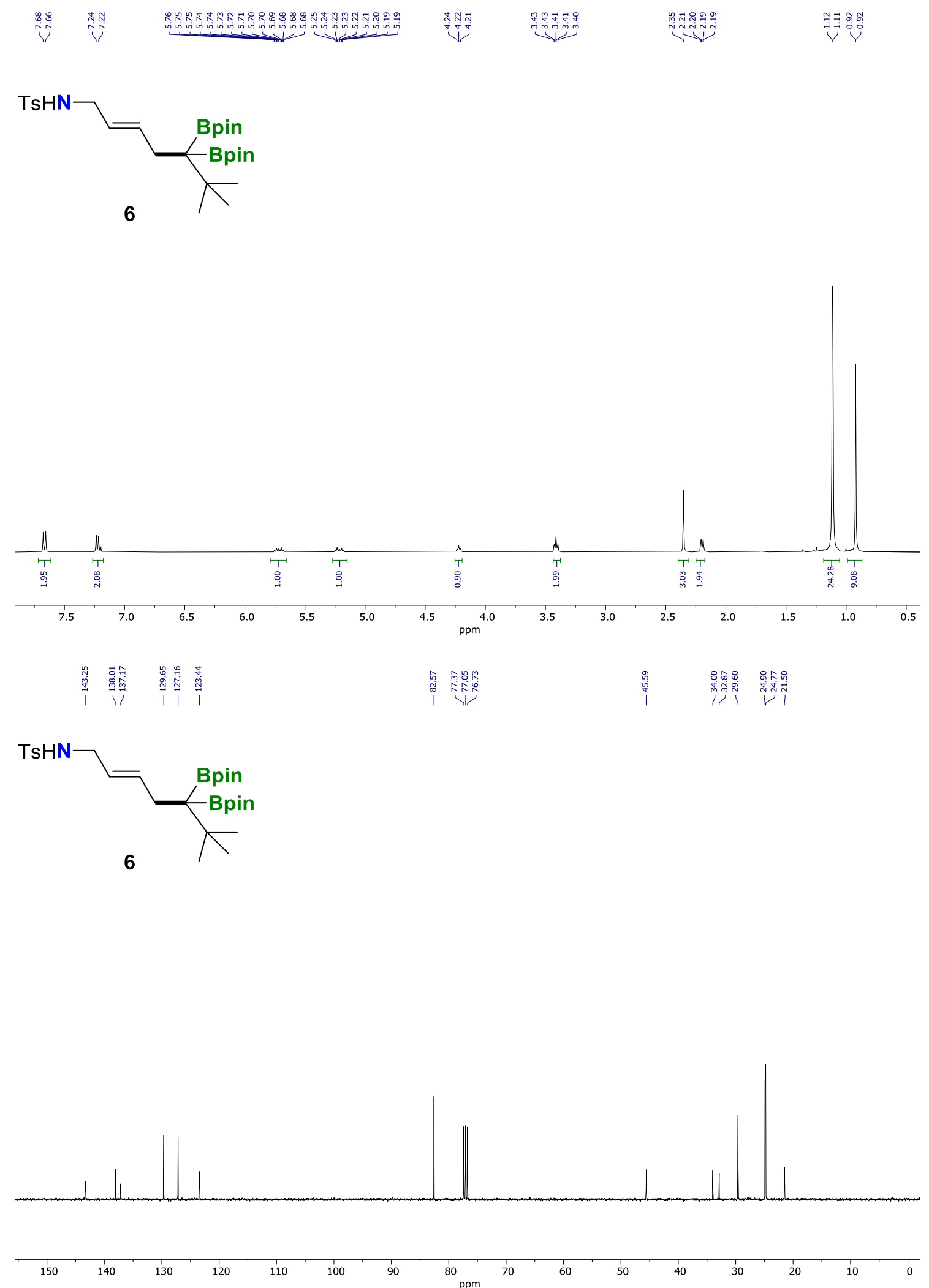

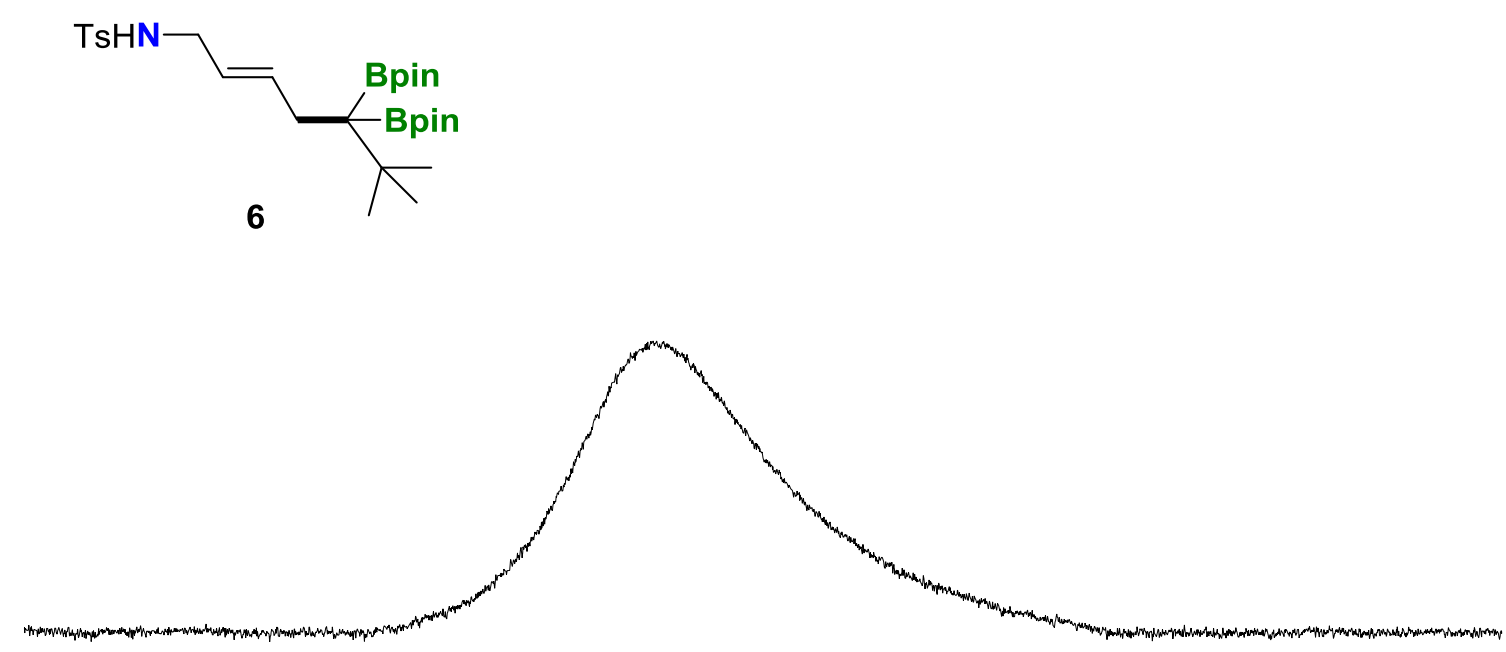

\begin{tabular}{lllllllllllllllllllllllllllllllllllllll}
\hline & 1 & 45 & 44 & 43 & 42 & 41 & 40 & 39 & 38 & 37 & 36 & 35 & 34 & 33 & 32 & 31 & 30 & 29 & 28 & 27 & 26 & 25 & 24 & 23 & 22 & 21 & 20 & 19 & 18 & 17 & 16 \\
\hline
\end{tabular}

诲
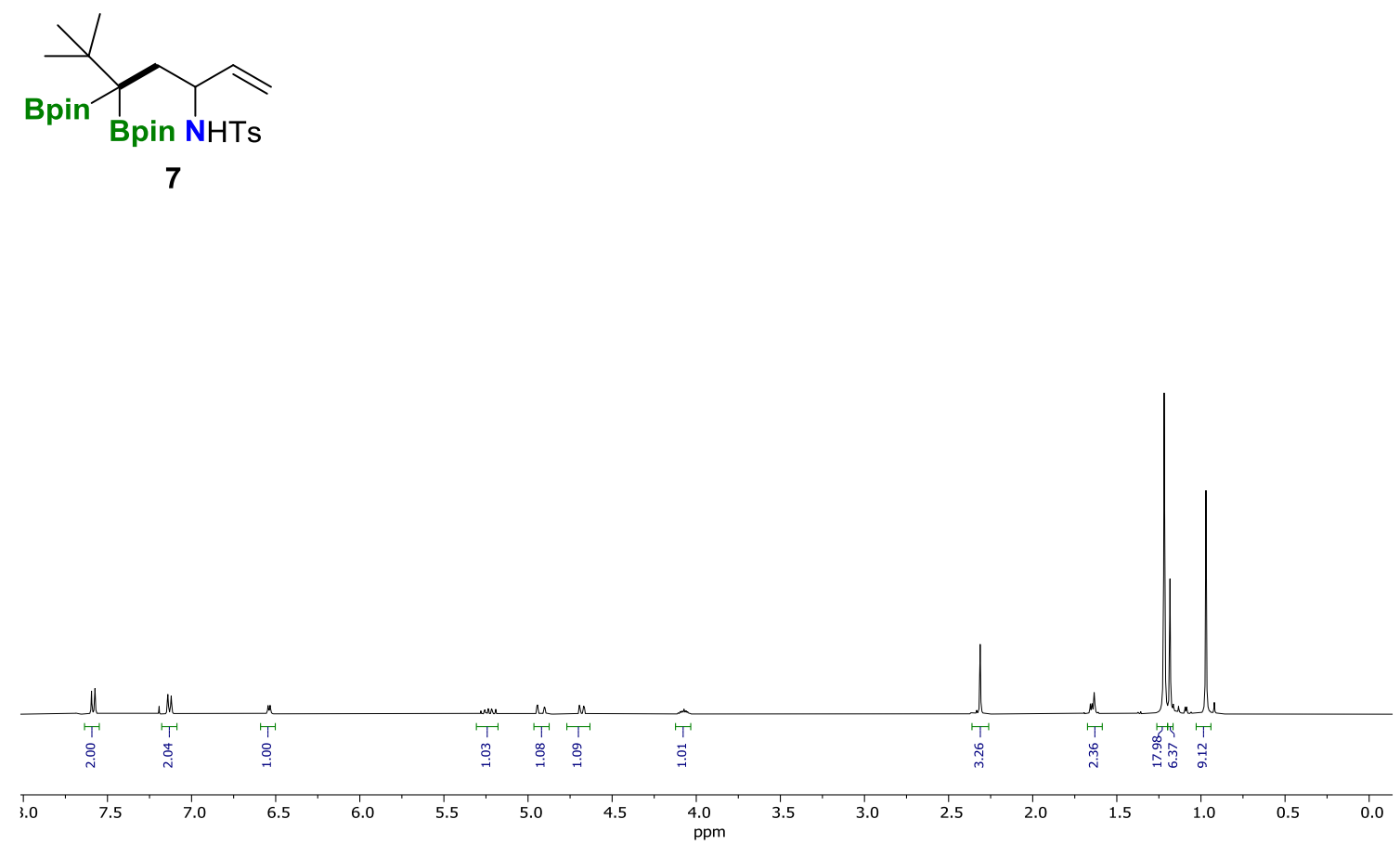

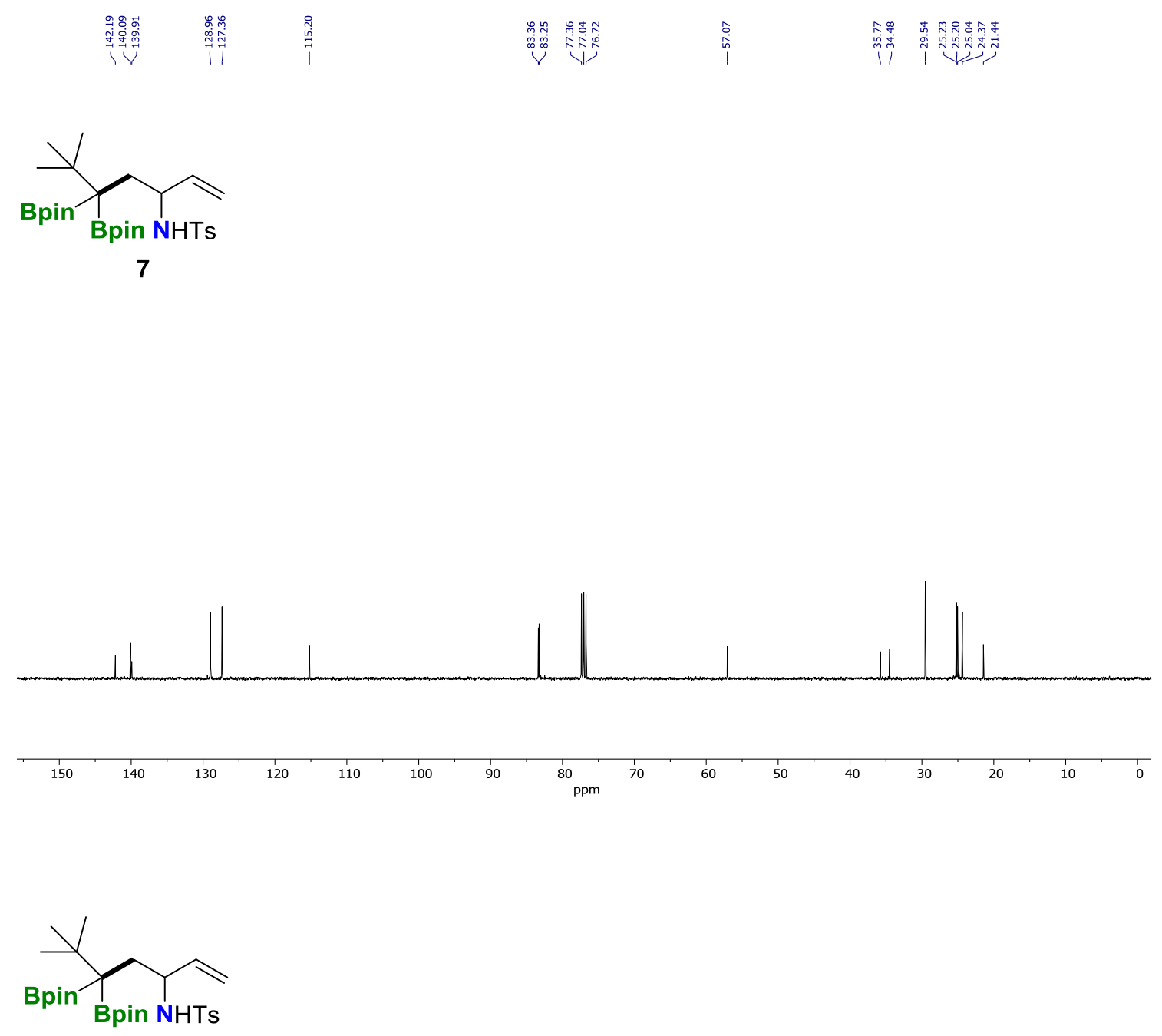

7

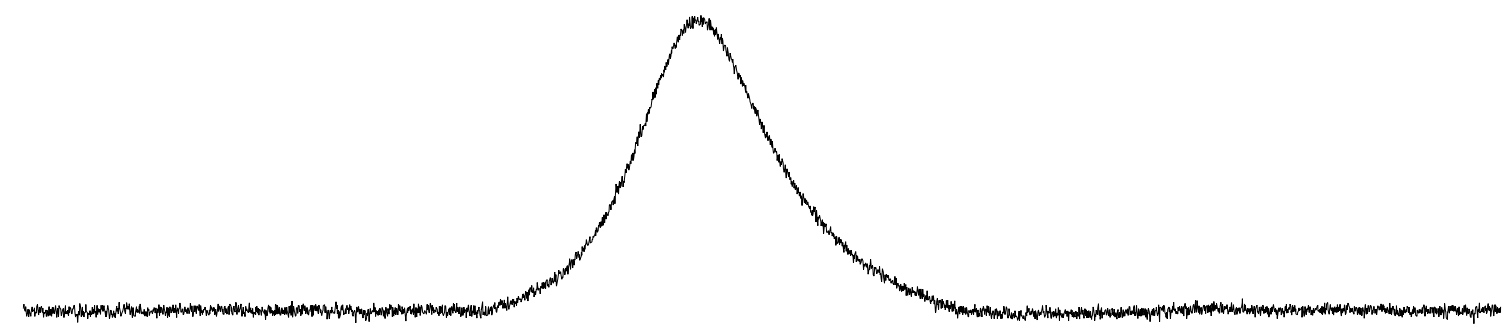



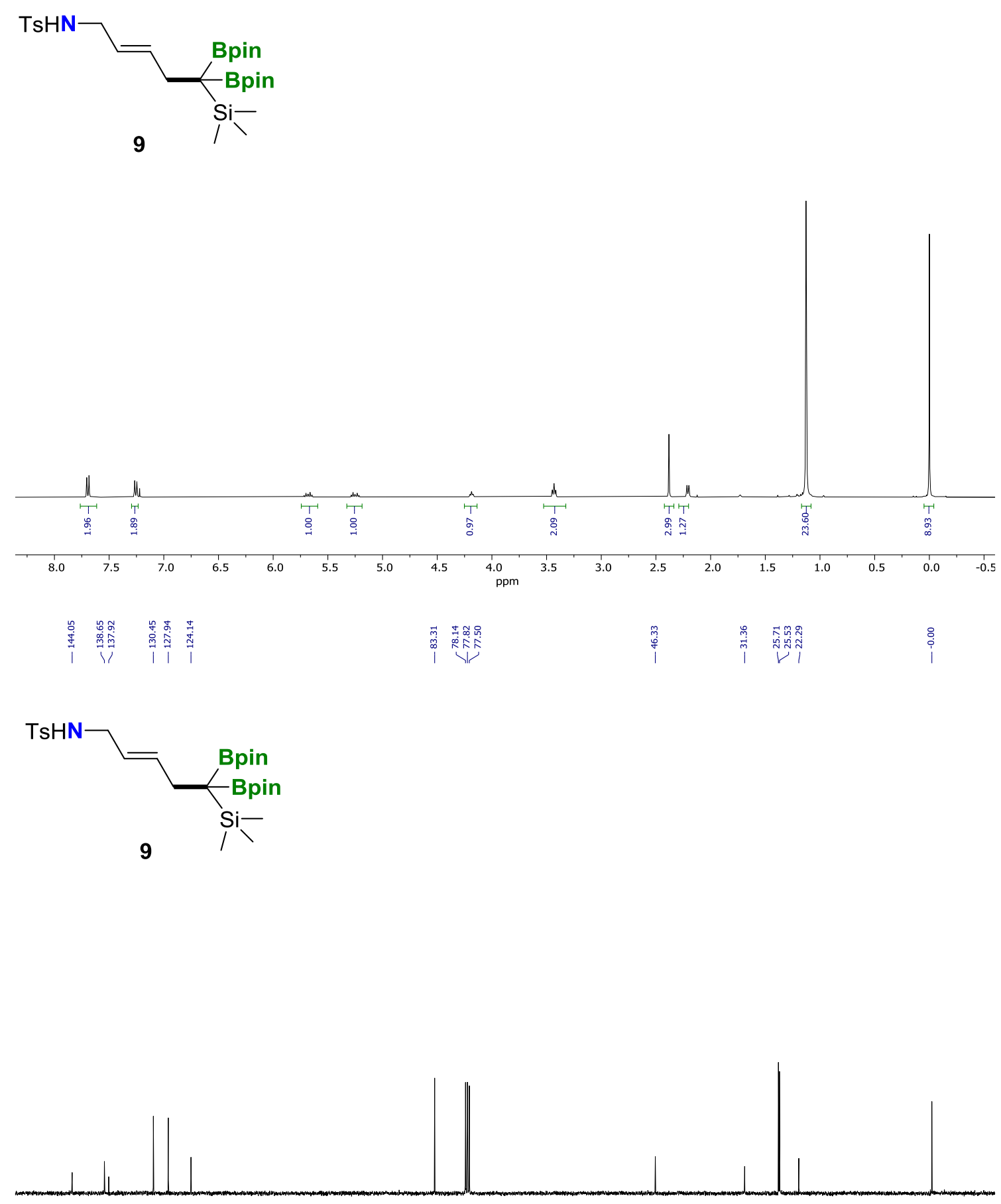

$\begin{array}{lllllll}150 & 140 & 130 & 120 & 110 & 100 & 90\end{array}$

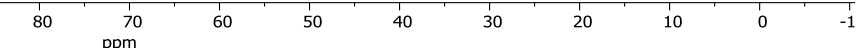




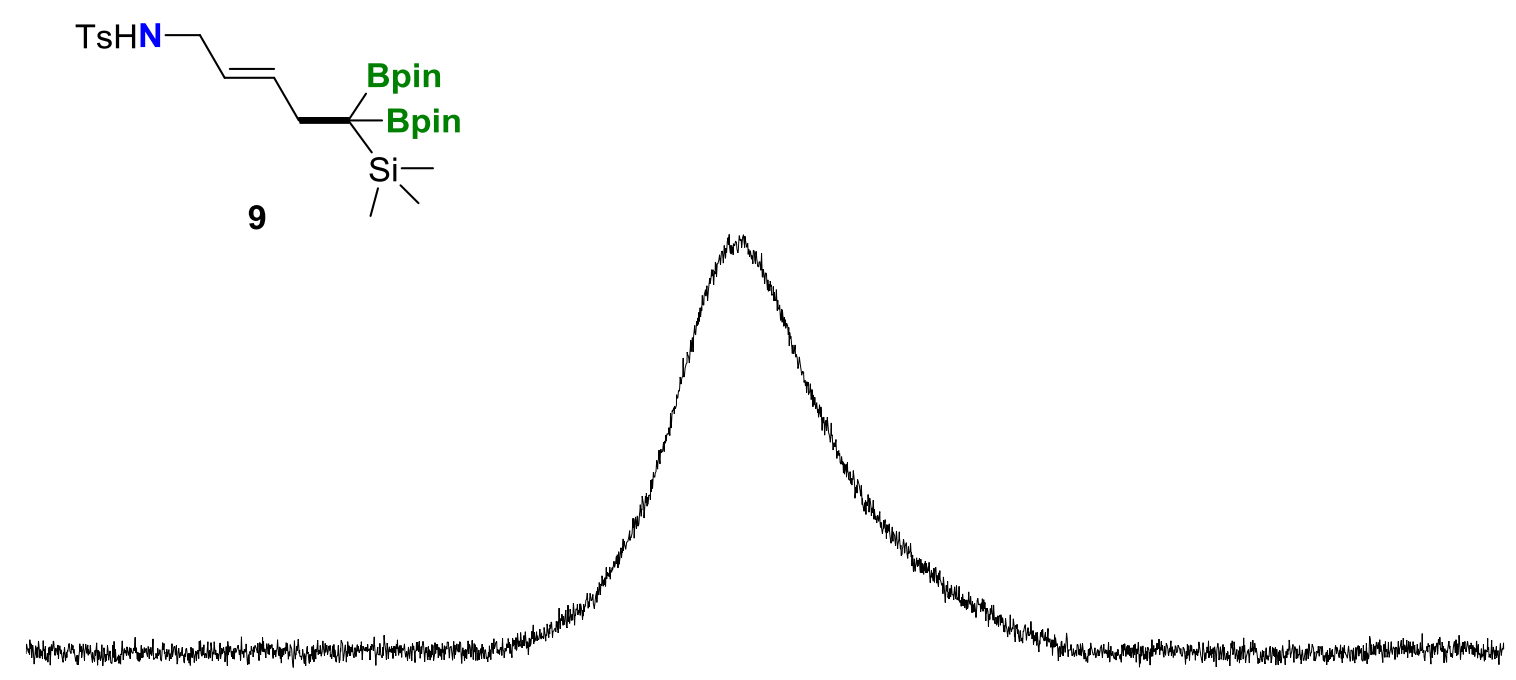

$\begin{array}{lllllllllllllllllllllllllllllllllllllllllllllll}1 & 1 & 50 & 49 & 48 & 4 & 46 & 45 & 14 & 43 & 42 & 14 & 40 & 39 & 38 & 37 & 36 & 35 & 34 & 33 & 32 & 31 & 30 & 29 & 28 & 1 & 26 & 25 & 14 & 12 & 12 & 1 & 10 & 1 & 1 & 17 & 16 & 15 & 14\end{array}$

謟<smiles>[B]C([B]NC(C)=S)(CC([Pb])C=C)[Si](C)(C)C</smiles>

10

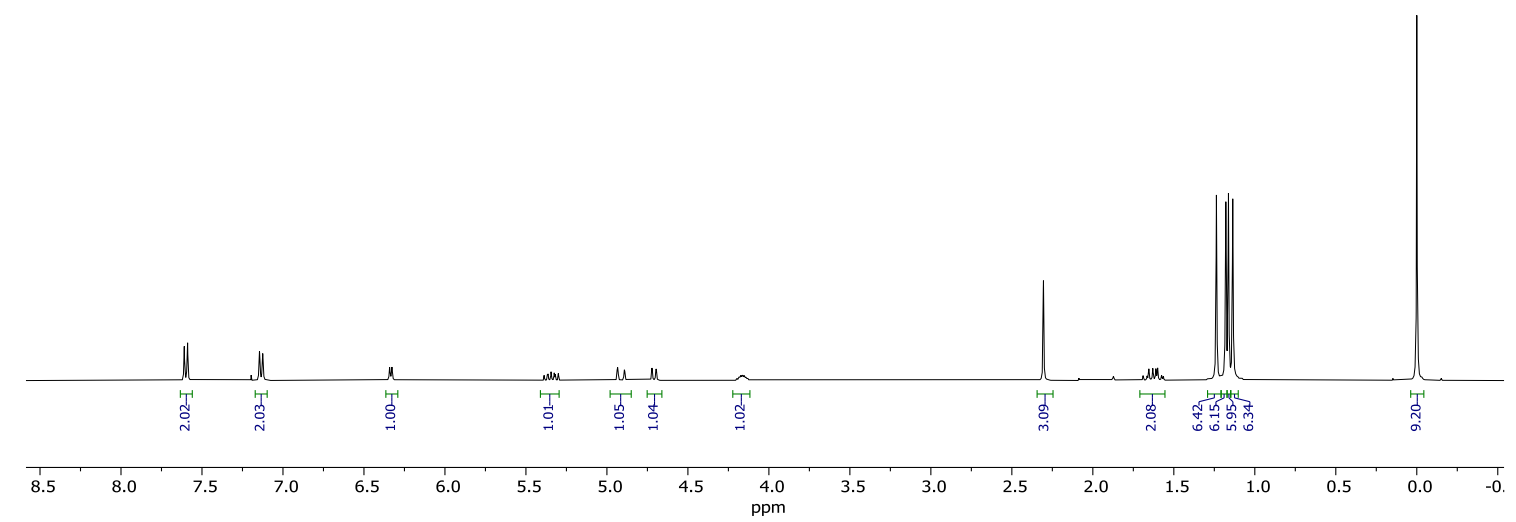




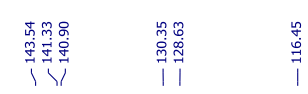

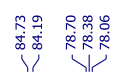

弯

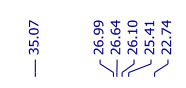

${ }_{B p i n}^{\prime} \overbrace{B p i n}^{S_{N H T s}}$

10

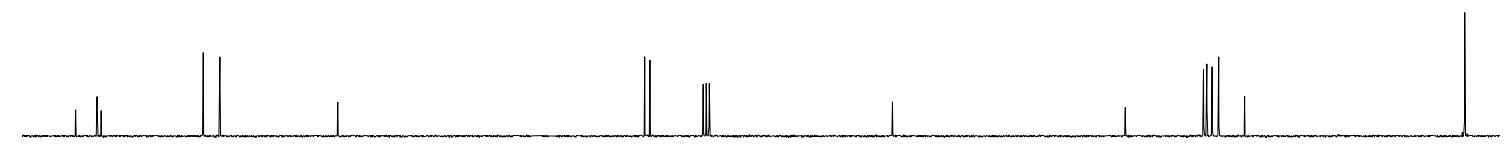

$\begin{array}{lllllllllllllllllllllllllllllllllllllll}145 & 140 & 135 & 130 & 125 & 120 & 115 & 110 & 105 & 100 & 95 & 90 & 85 & 80 & 75 & 70 & 65 & 60 & 55 & 50 & 45 & 40 & 35 & 30 & 25 & 20 & 15 & 10 & 5 & 0\end{array}$

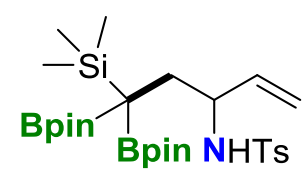

10

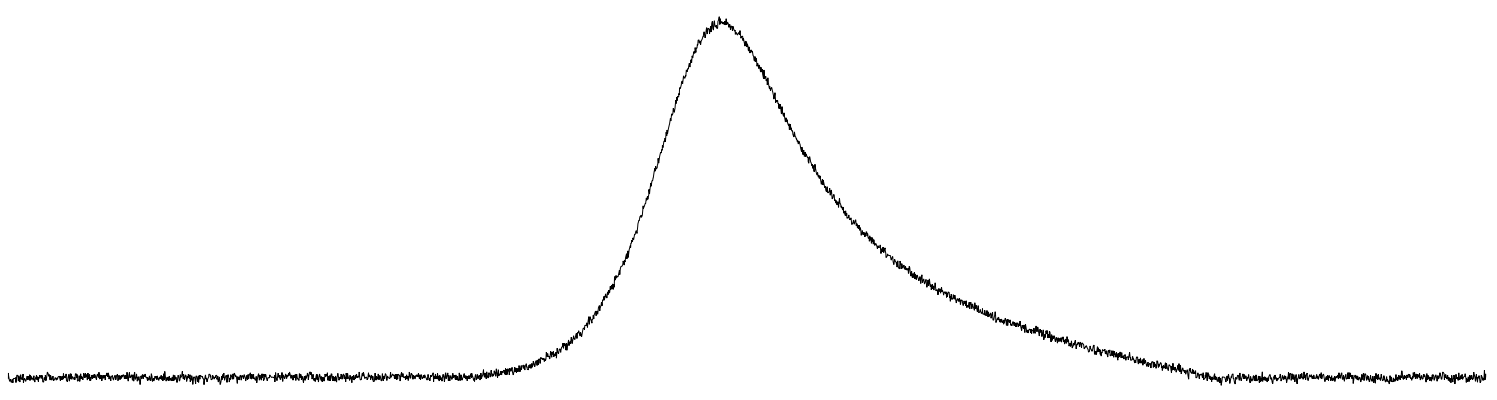

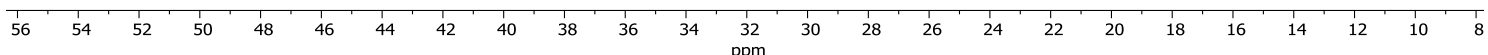




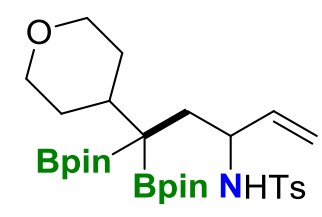

12

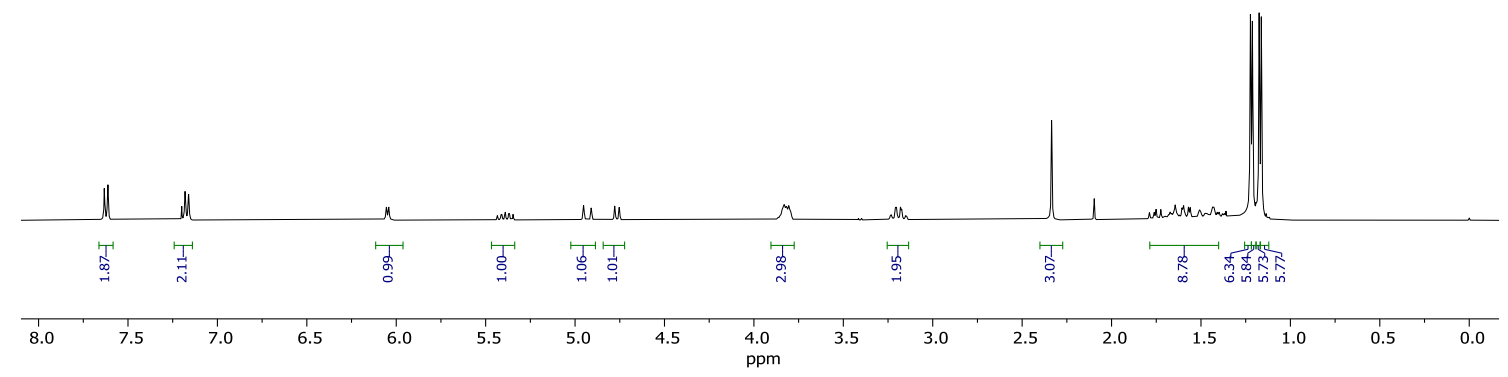

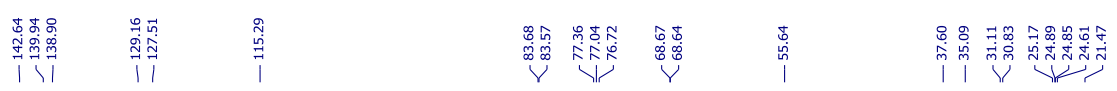

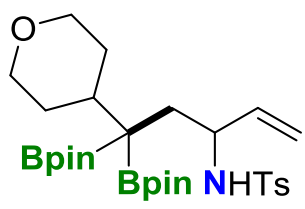

12
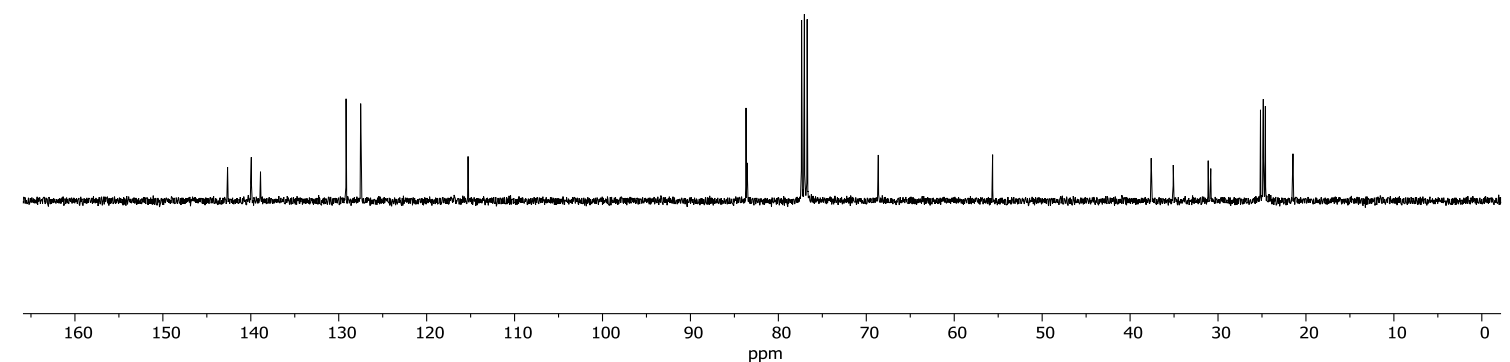


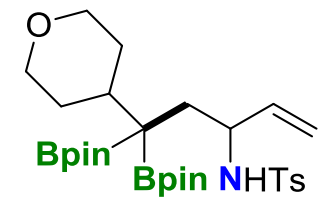

12
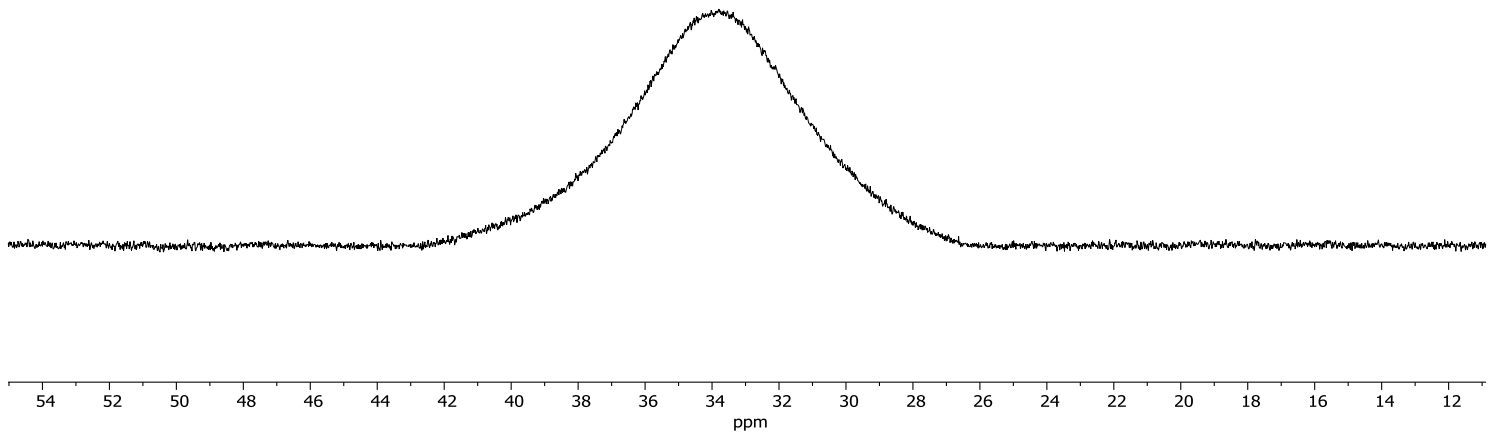

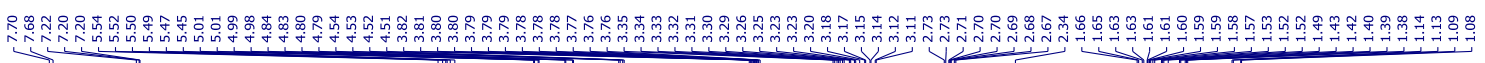<smiles>[3H]NCC(C=C)C([Pb]c1ccccc1)(C1CCOCC1)C1CCOCC1</smiles>

13

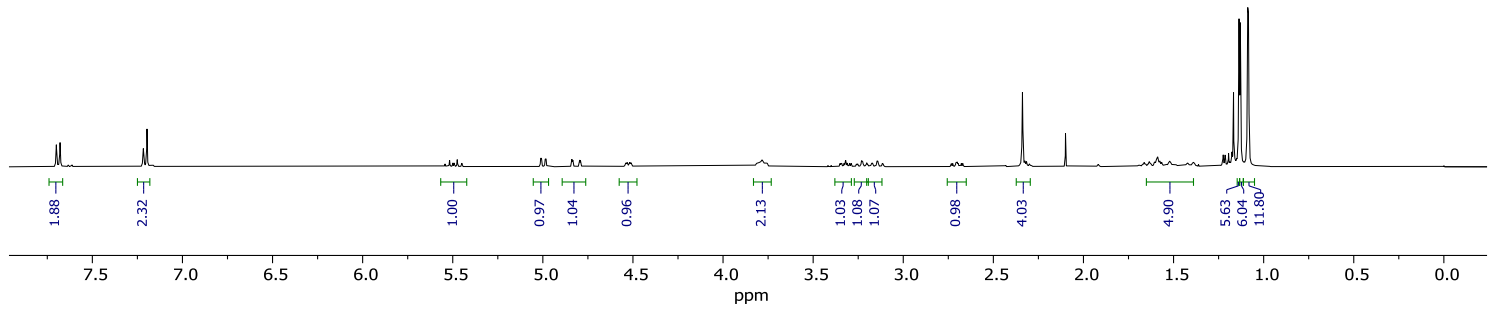




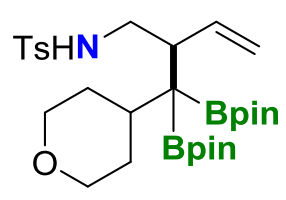

13
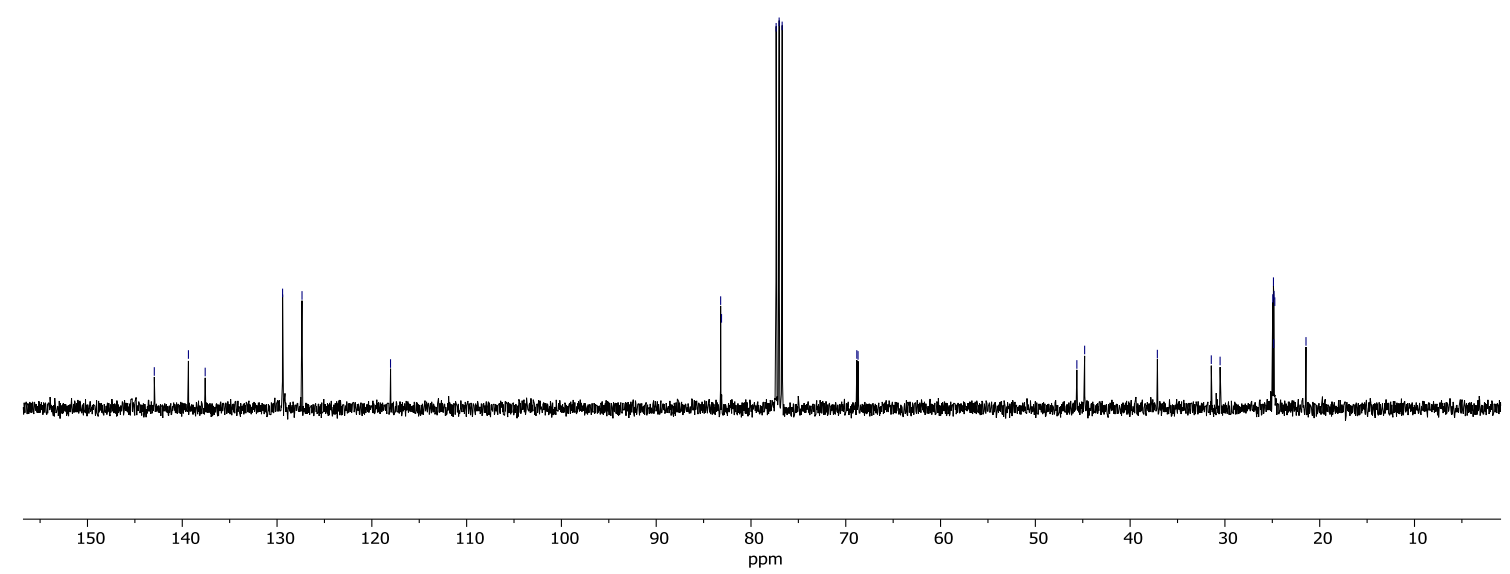<smiles>[3H]NCC(C=C)C([Pb])([Pb])C1CCOCC1</smiles>

13

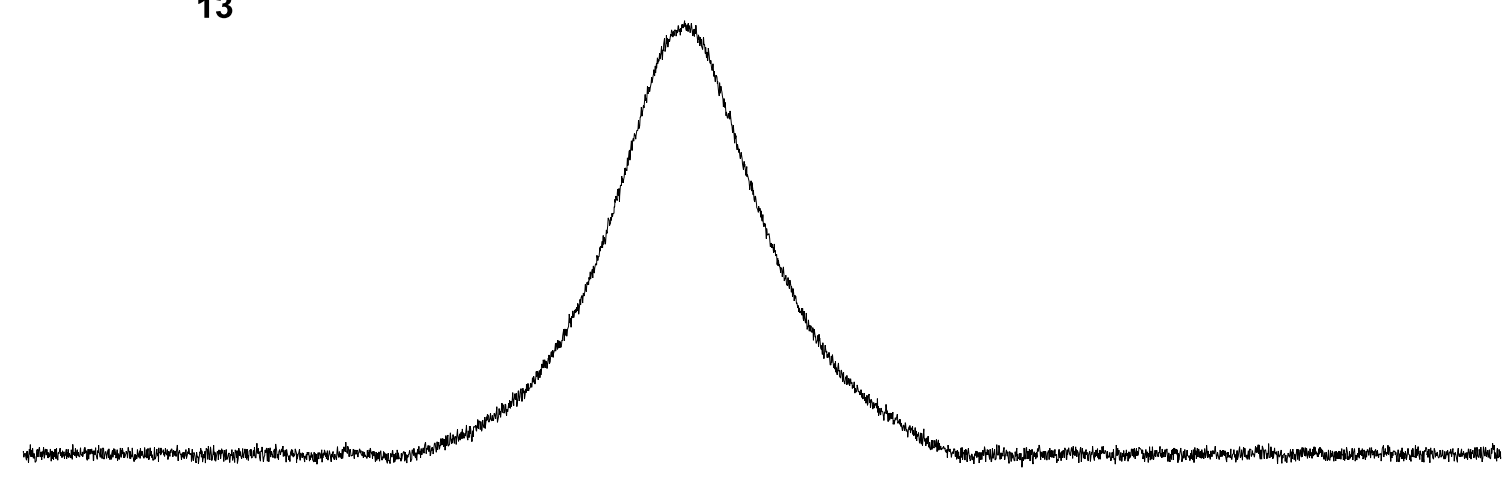




\section{(CA月员) \\ Bpin \\ Bpin NHTs}

15

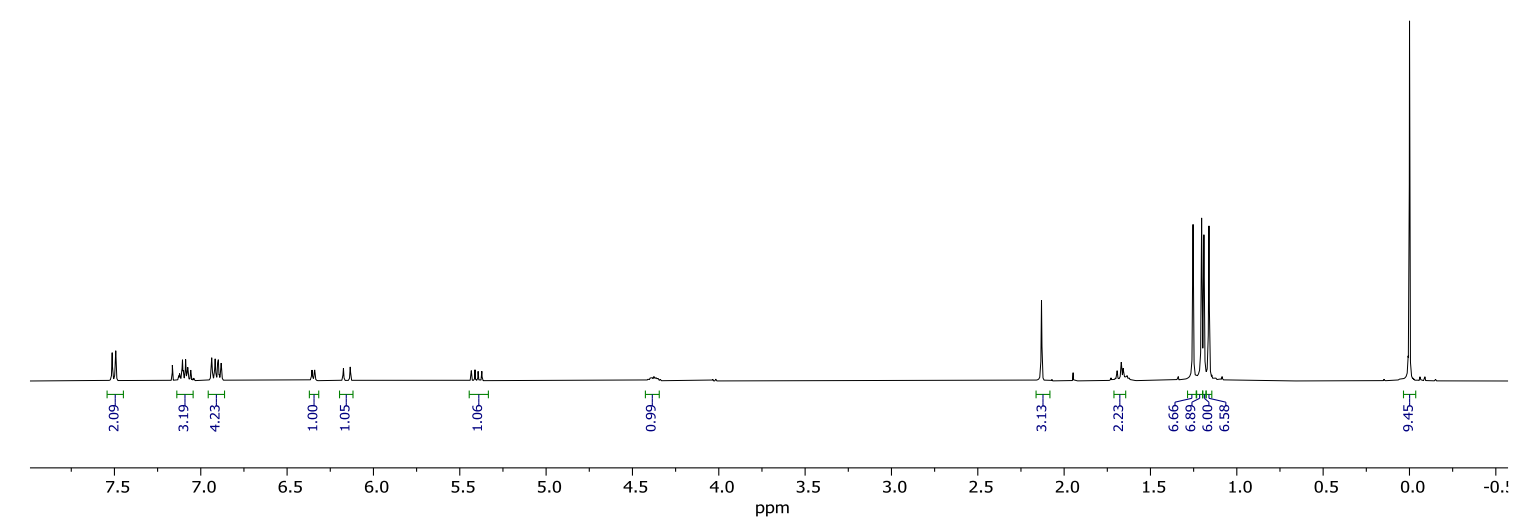

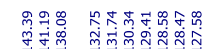

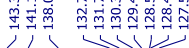

$\left(\mathrm{CH}_{3}\right)_{3} \mathrm{Si} \underbrace{}_{\text {Bpin }} \underbrace{\mathrm{Ph}}_{\mathrm{NHTs}}$

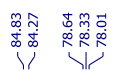

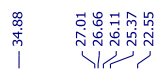

15

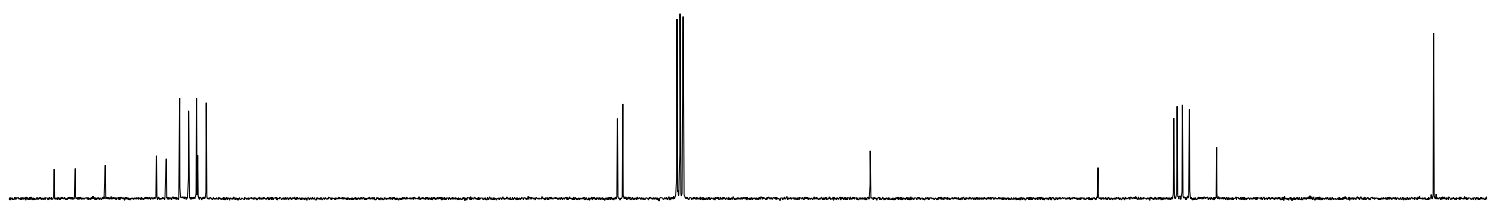

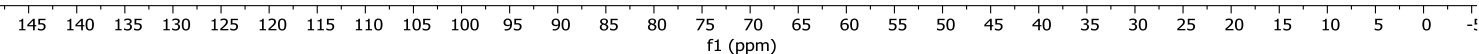


$\mathrm{Bpin}_{\mathrm{Bpin} \mathrm{NHTs}_{3} \mathrm{Si}}^{\mathrm{Ph}}$

15

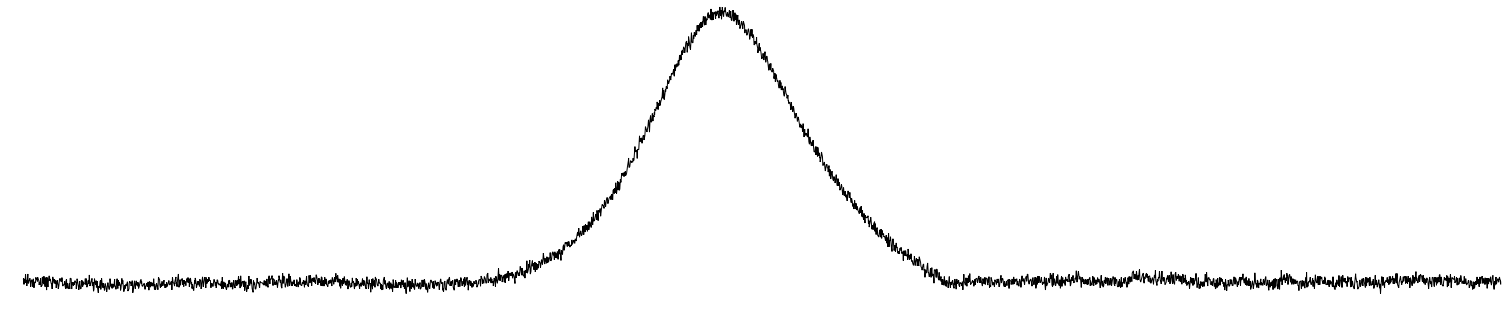

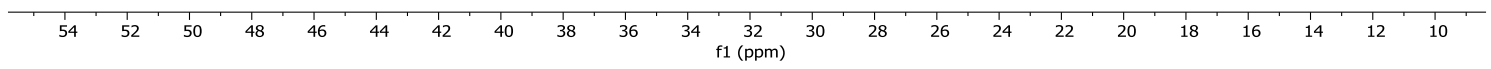

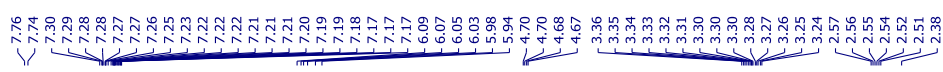

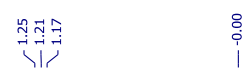<smiles>[AlH2]NCC(/C=C/c1ccccc1)C([Si])([Si])C(Br)(Br)c1ccccc1</smiles>

16

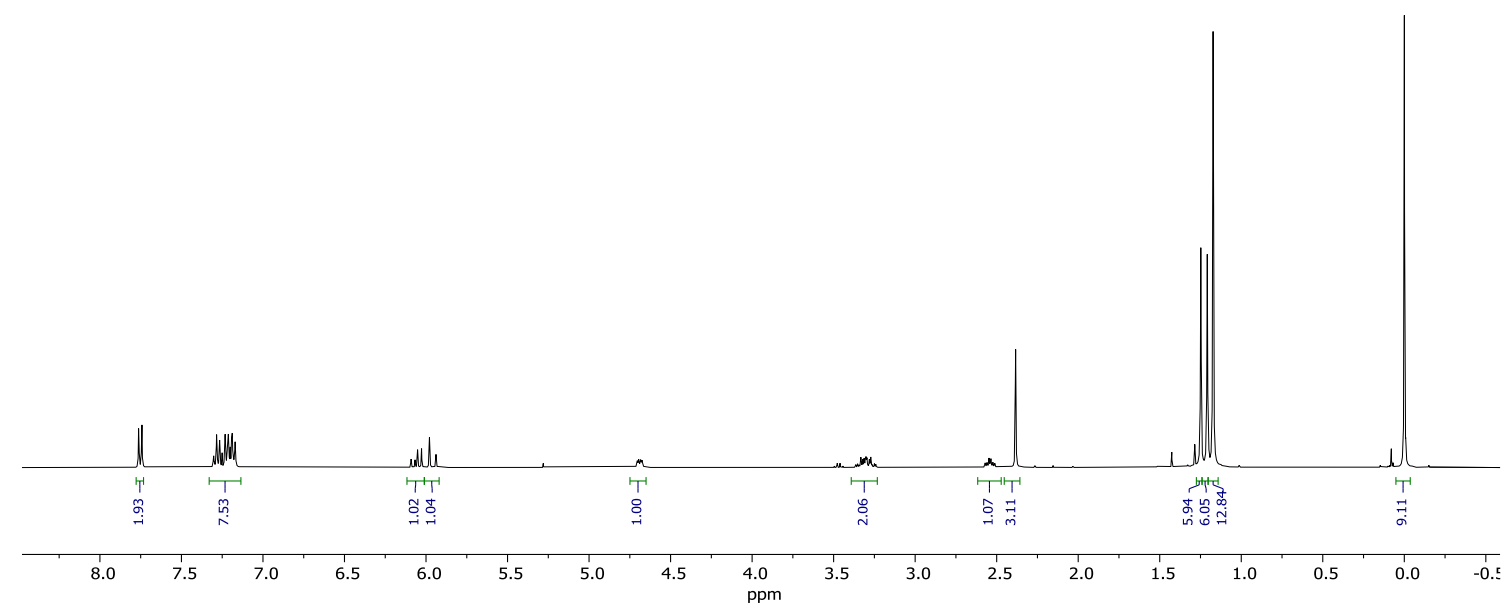




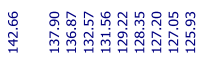

1

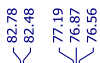

站亭

หัง

पूरूก

$\overbrace{\left(\mathrm{CH}_{3}\right)_{3} \mathrm{Si}}^{\mathrm{TsHN}} \mathrm{Ph}$

16
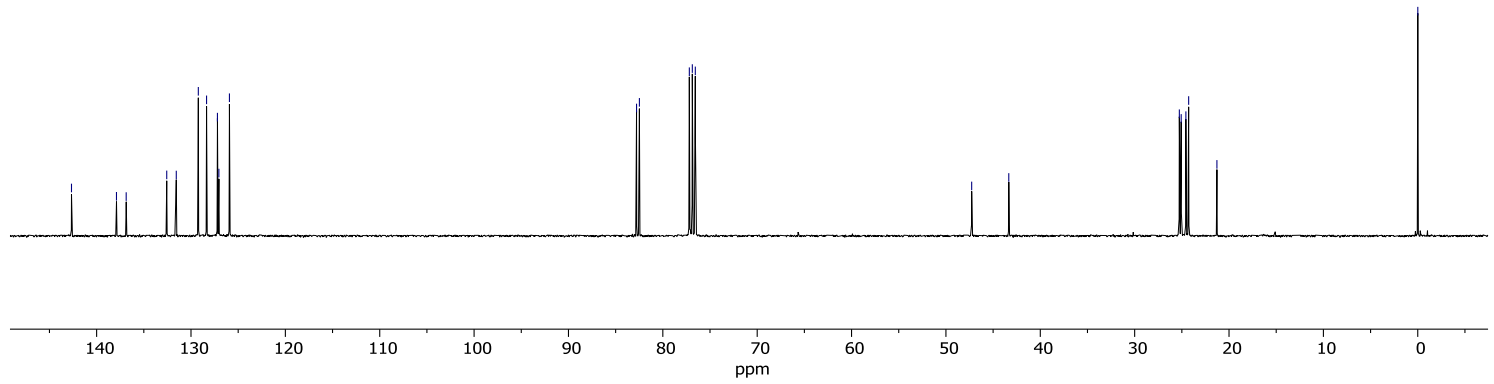

$$
\overbrace{\substack{\text { Bpin } \\ 16}}^{\mathrm{C}}
$$

16

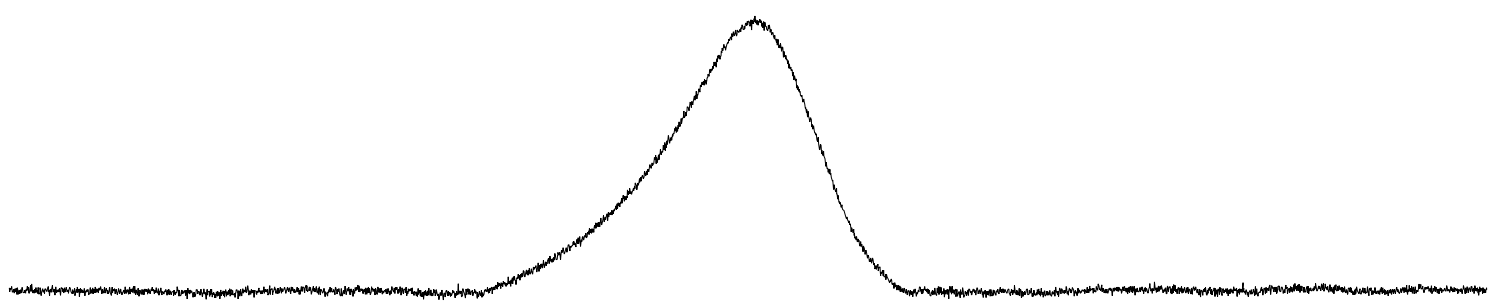

$\begin{array}{llllllllllllllllllllllllllllllllllllllllllllll}62 & 60 & 58 & 56 & 54 & 52 & 50 & 48 & 46 & 44 & 42 & 40 & 38 & 36 & 34 & 32 & 30 & 28 & 26 & 24 & 22 & 20 & 18 & 16 & 14 & 12 & 10 & 8 & \epsilon\end{array}$ 
<smiles>[10BH2]C(CC([10BH2])([10BH2])[13CH3])/C(=C/C)c1ccccc1</smiles>

18

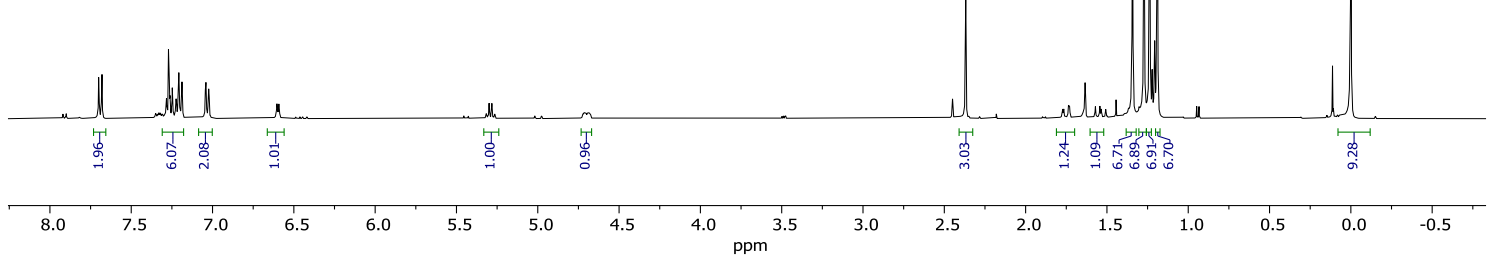

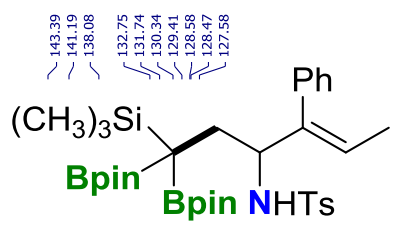

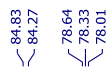

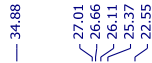

18

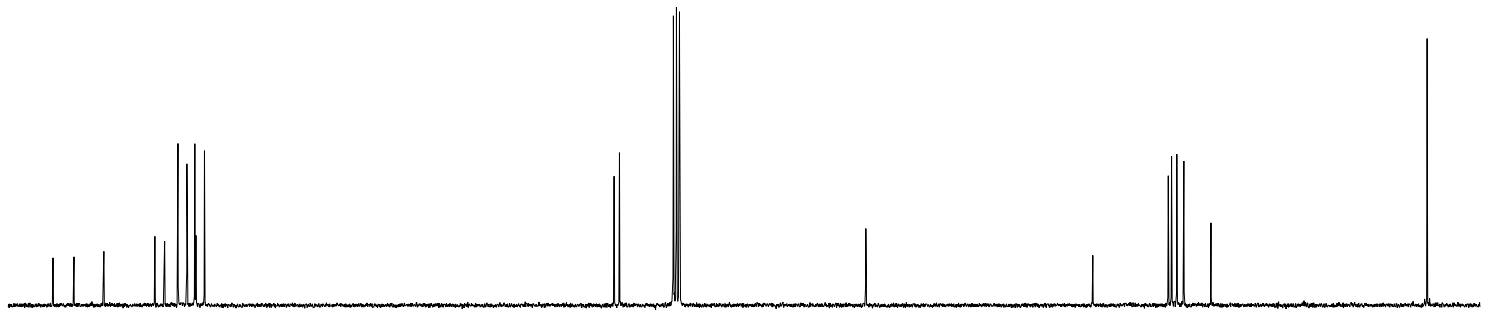

$\begin{array}{lllllllllllllllllllllllllllllllllllllllllllll}145 & 140 & 135 & 130 & 125 & 120 & 115 & 110 & 105 & 100 & 95 & 90 & 85 & 80 & 75 & 70 & 65 & 60 & 55 & 50 & 45 & 40 & 35 & 30 & 25 & 20 & 15 & 10 & 5 & 0 & -1\end{array}$ 


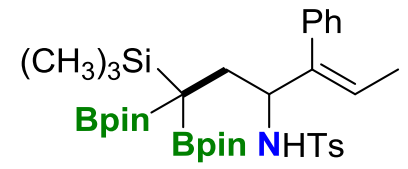

18

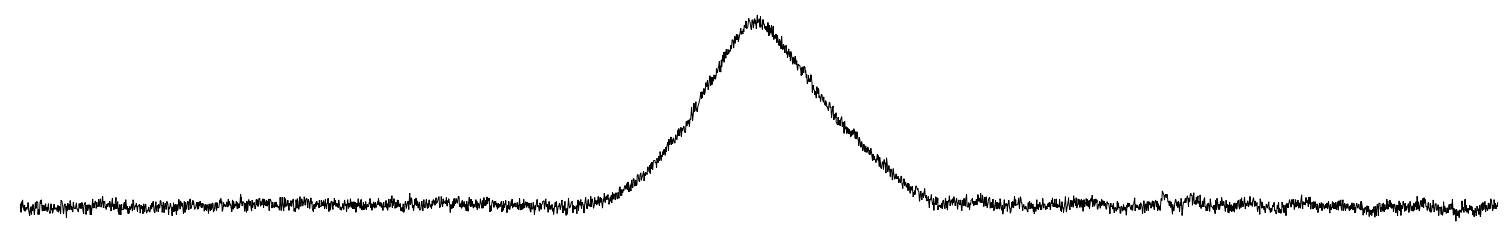

$\begin{array}{llllllllllllllllllllllllllllllllll}56 & 54 & 52 & 50 & 48 & 46 & 44 & 42 & 40 & 38 & 36 & 34 & 32 & 30 & 28 & 16 & 24 & 22 & 20 & 18 & 16 & 14 & 12 & 10\end{array}$

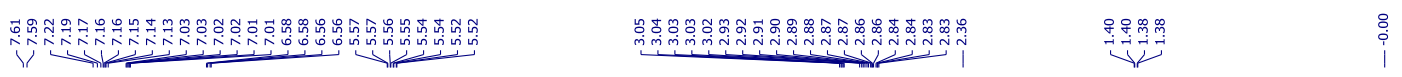

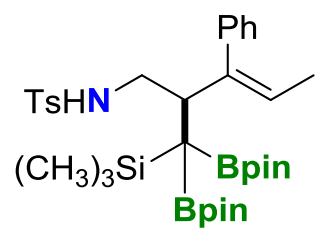

19

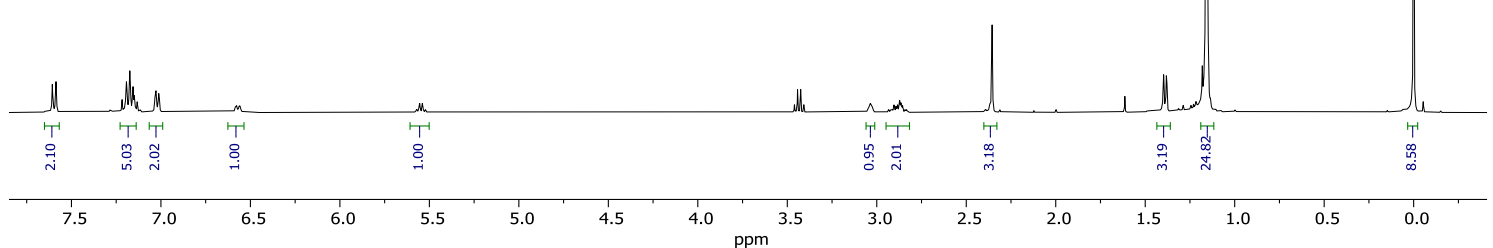




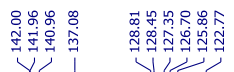

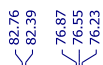

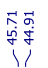

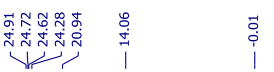

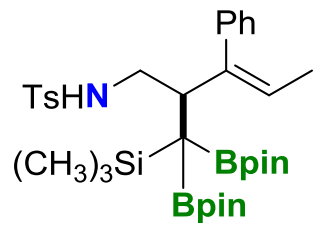

19
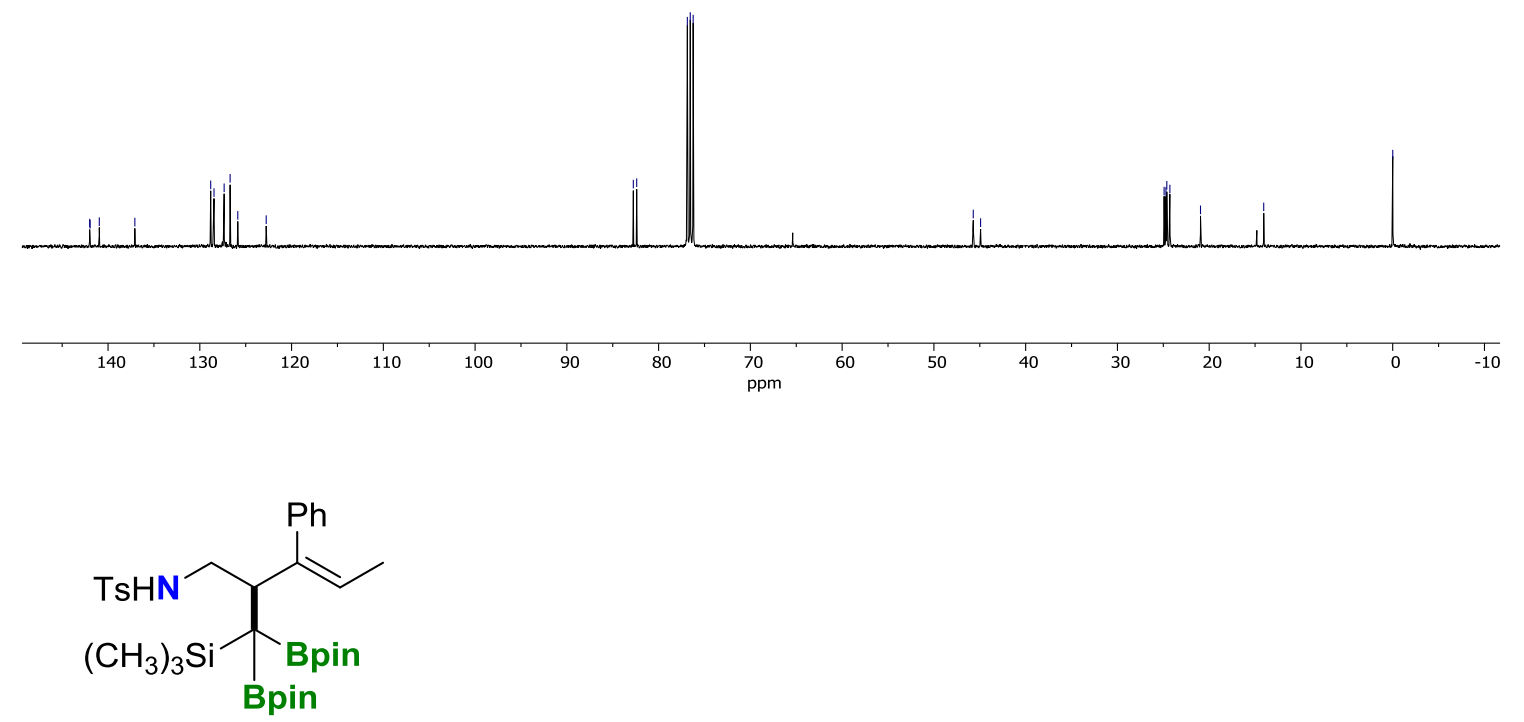

19

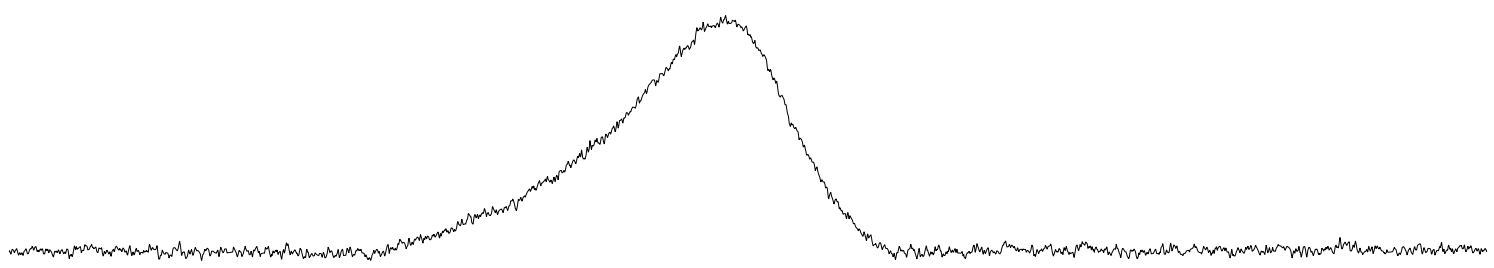



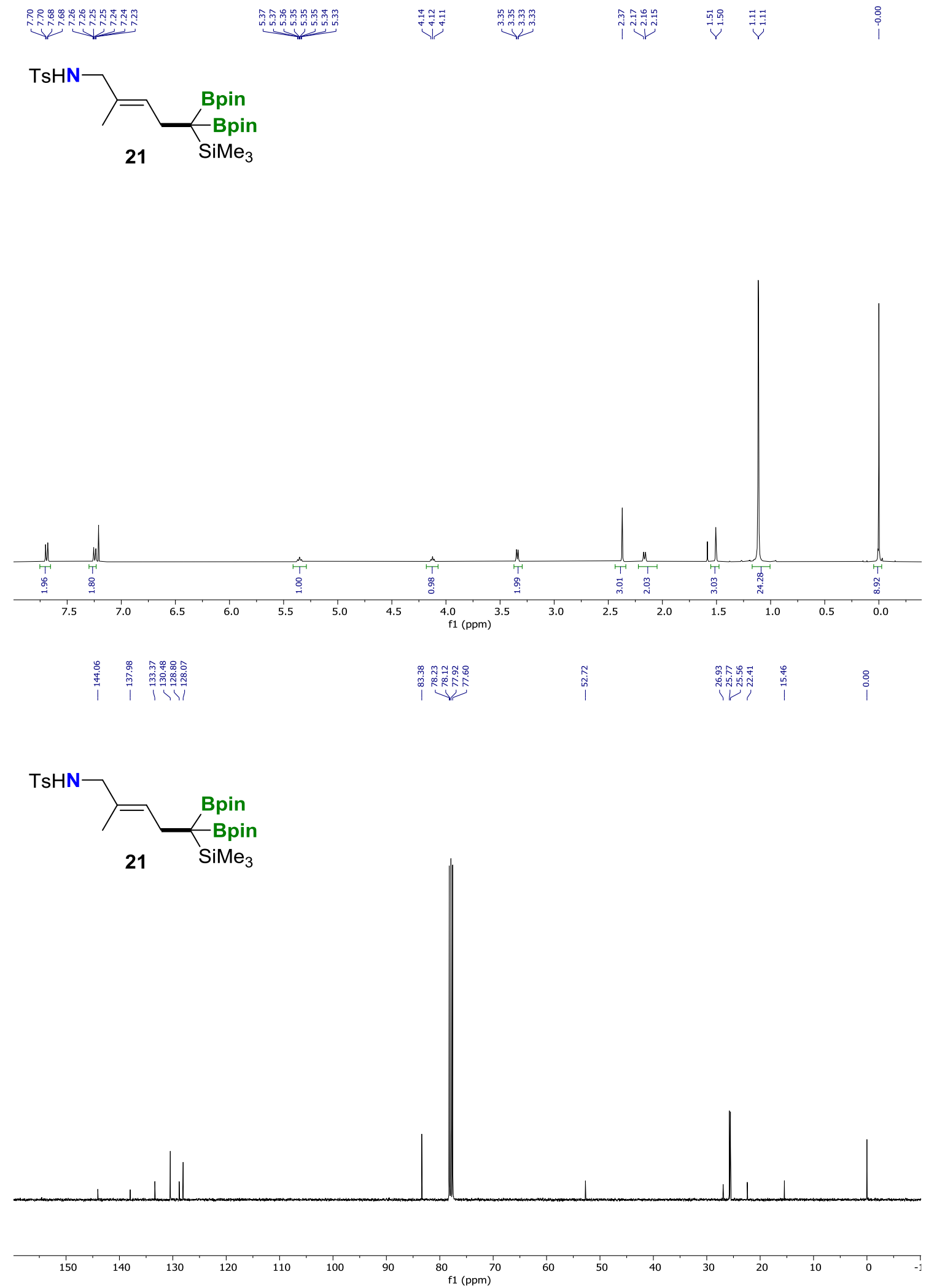

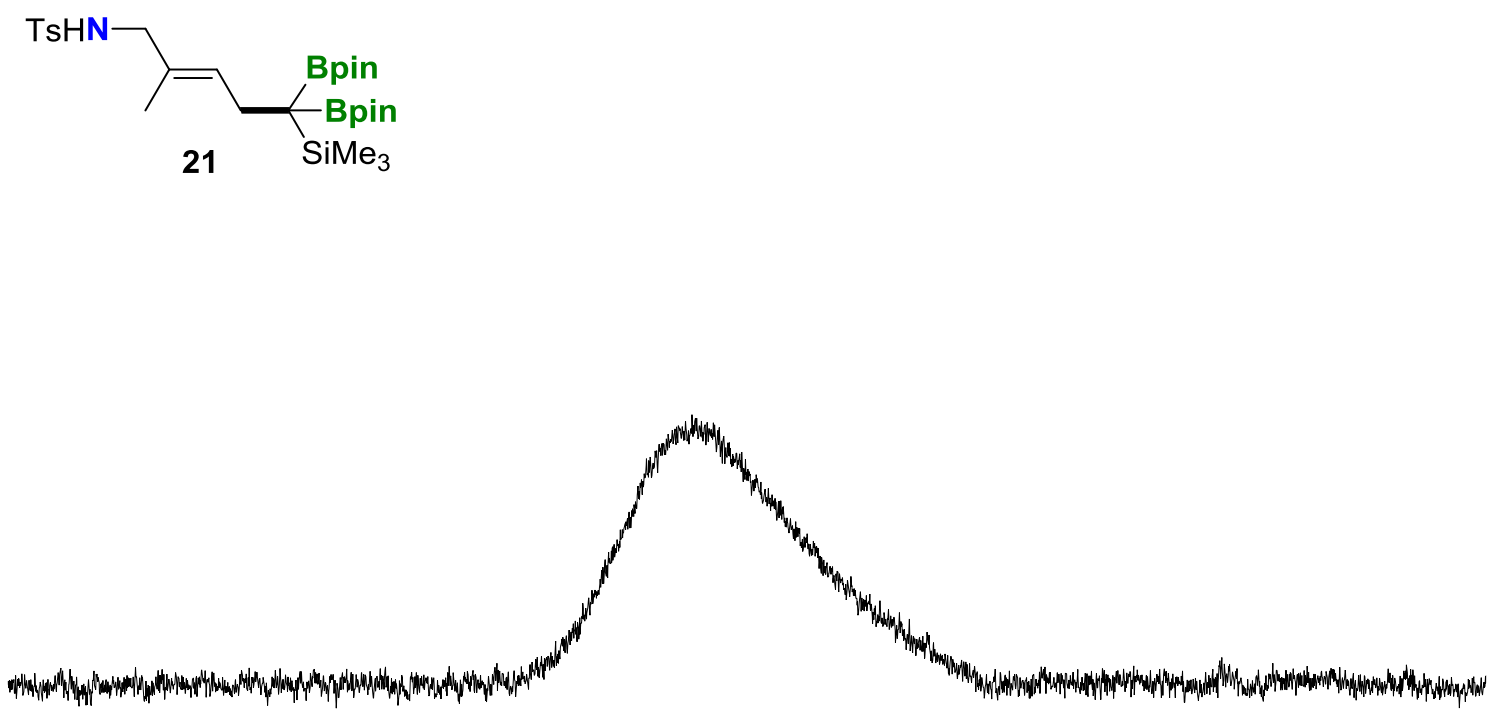
$\begin{array}{lllllllllllllllllllllllllllllllllllllllllllllllll}49 & 48 & 47 & 46 & 45 & 44 & 43 & 42 & 41 & 40 & 39 & 38 & 37 & 36 & 35 & 34 & 33 & 32 & 31 & 30 & 29 & 28 & 27 & 26 & 25 & 24 & 23 & 22 & 21 & 20 & 19 & 18 & 17 & 16 & 15 & 14\end{array}$

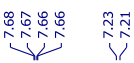

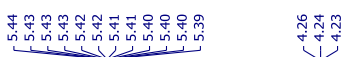

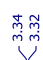

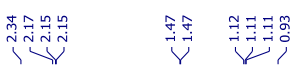

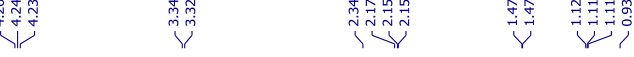

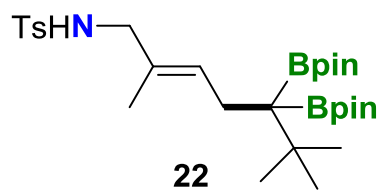

22

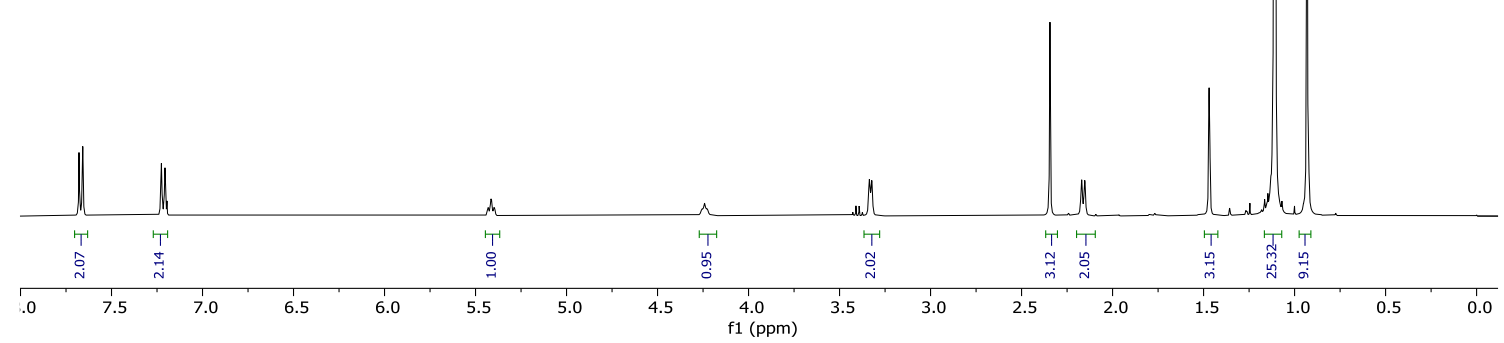




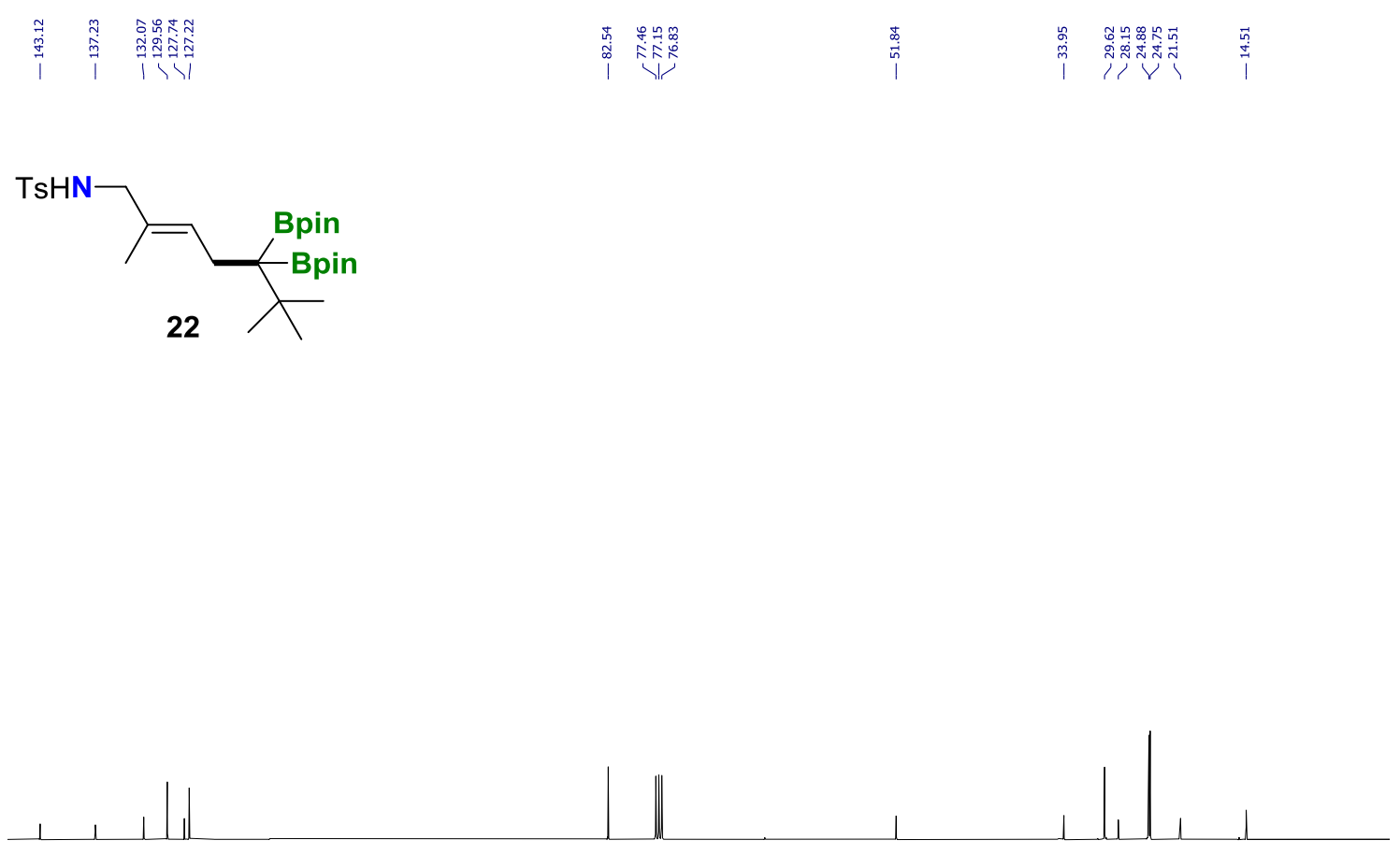

\begin{tabular}{lllllllllllllllllllllllllllllllllllllllll}
145 & 140 & 135 & 130 & 125 & 120 & 115 & 110 & 105 & 100 & 95 & 90 & 85 & 80 & 75 & 70 & 65 & 60 & 5 & 50 & 4 & 40 & 35 & 30 & 25 & 20 & 15 & 10 & 5 & 0 \\
\hline
\end{tabular}
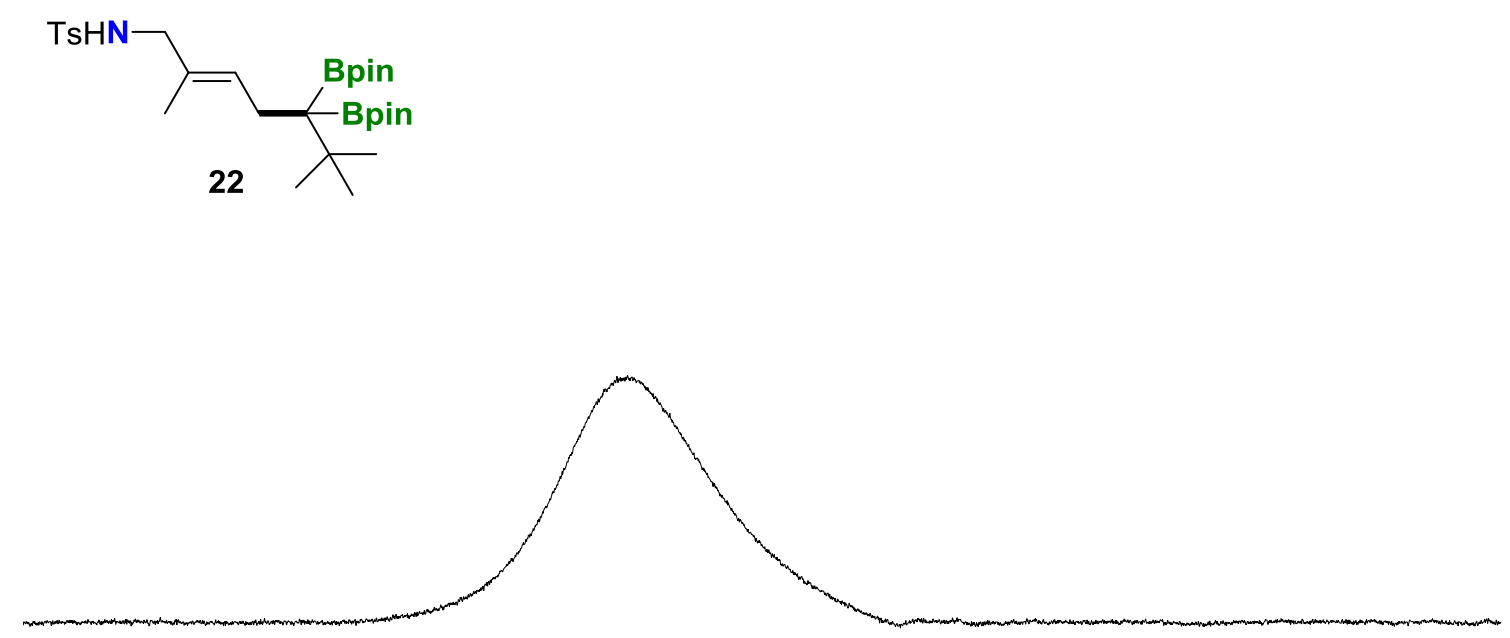

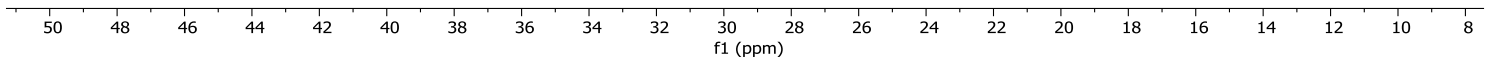




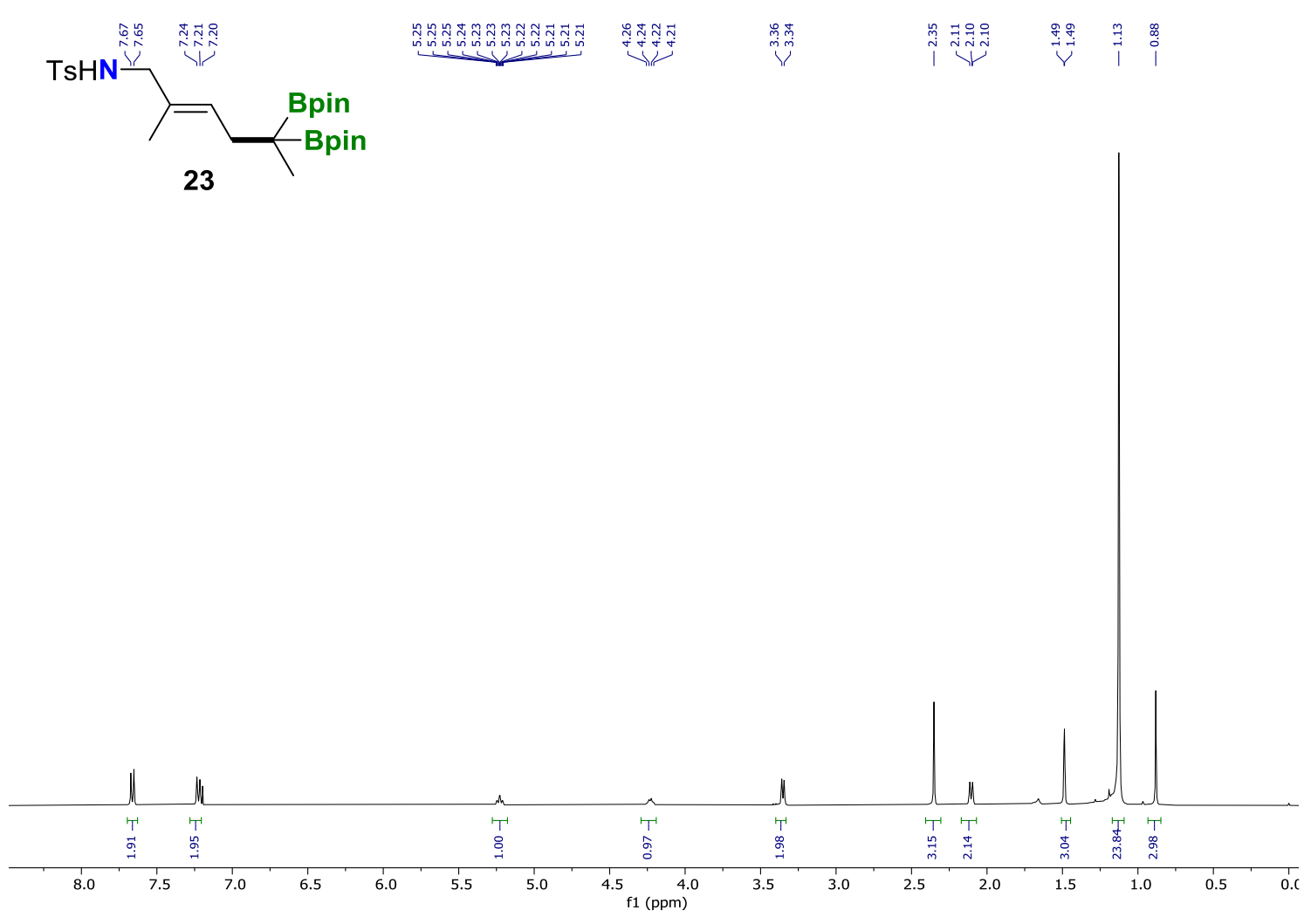

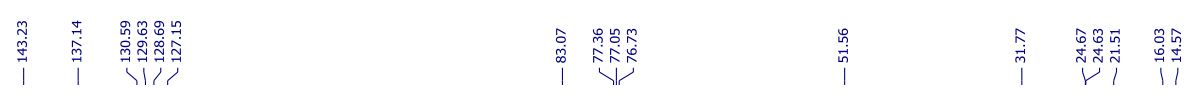

${ }_{23}^{\mathrm{TsHN}} \underbrace{\text { Bpin }}_{\text {Bpin }}$

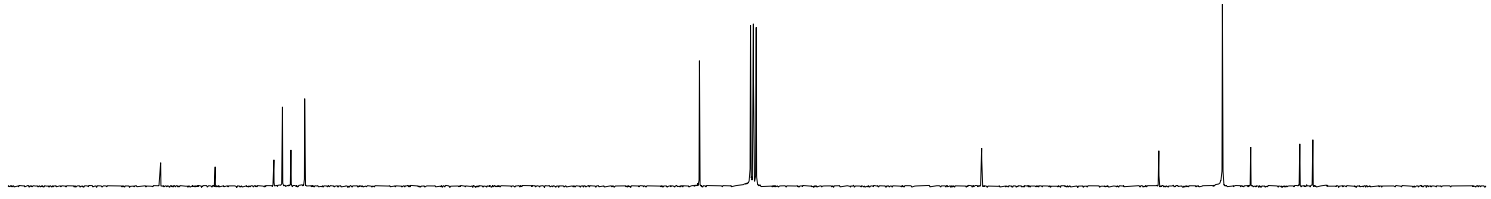

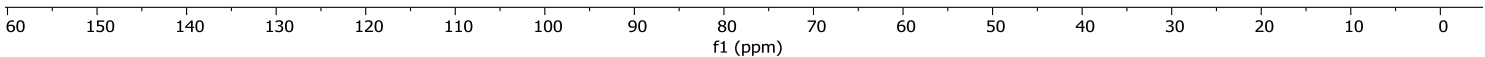


${ }_{23}^{\mathrm{TsHN}} \underbrace{\text { Bpin }}_{\text {Bpin }}$

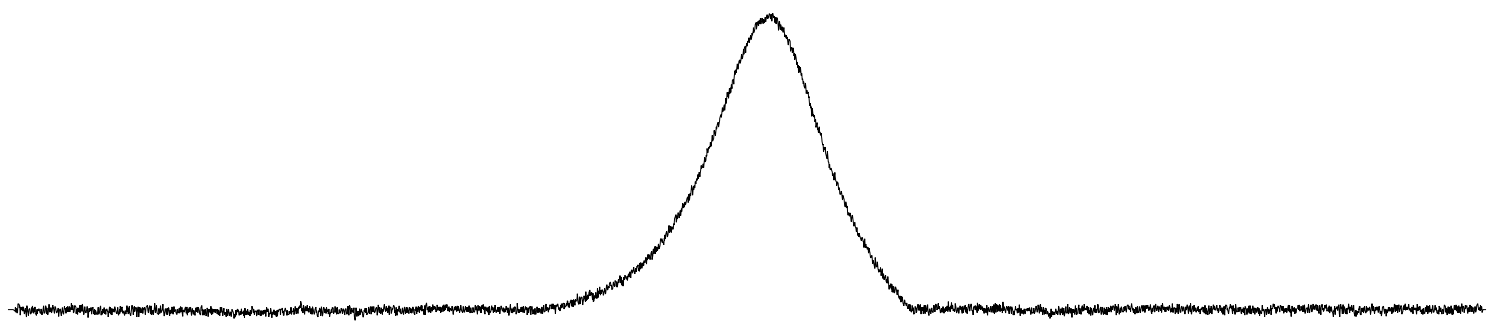

$\begin{array}{llllllllllllllllllllllllllllllllllllll}64 & 62 & 60 & 58 & 56 & 54 & 52 & 50 & 48 & 46 & 44 & 42 & 40 & 38 & 36 & 34 & 32 & 30 & 28 & 26 & 24 & 22 & 20 & 18 & 16 & 14 & 12 & 10 & 8 & 6\end{array}$

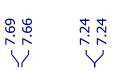

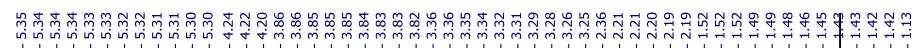

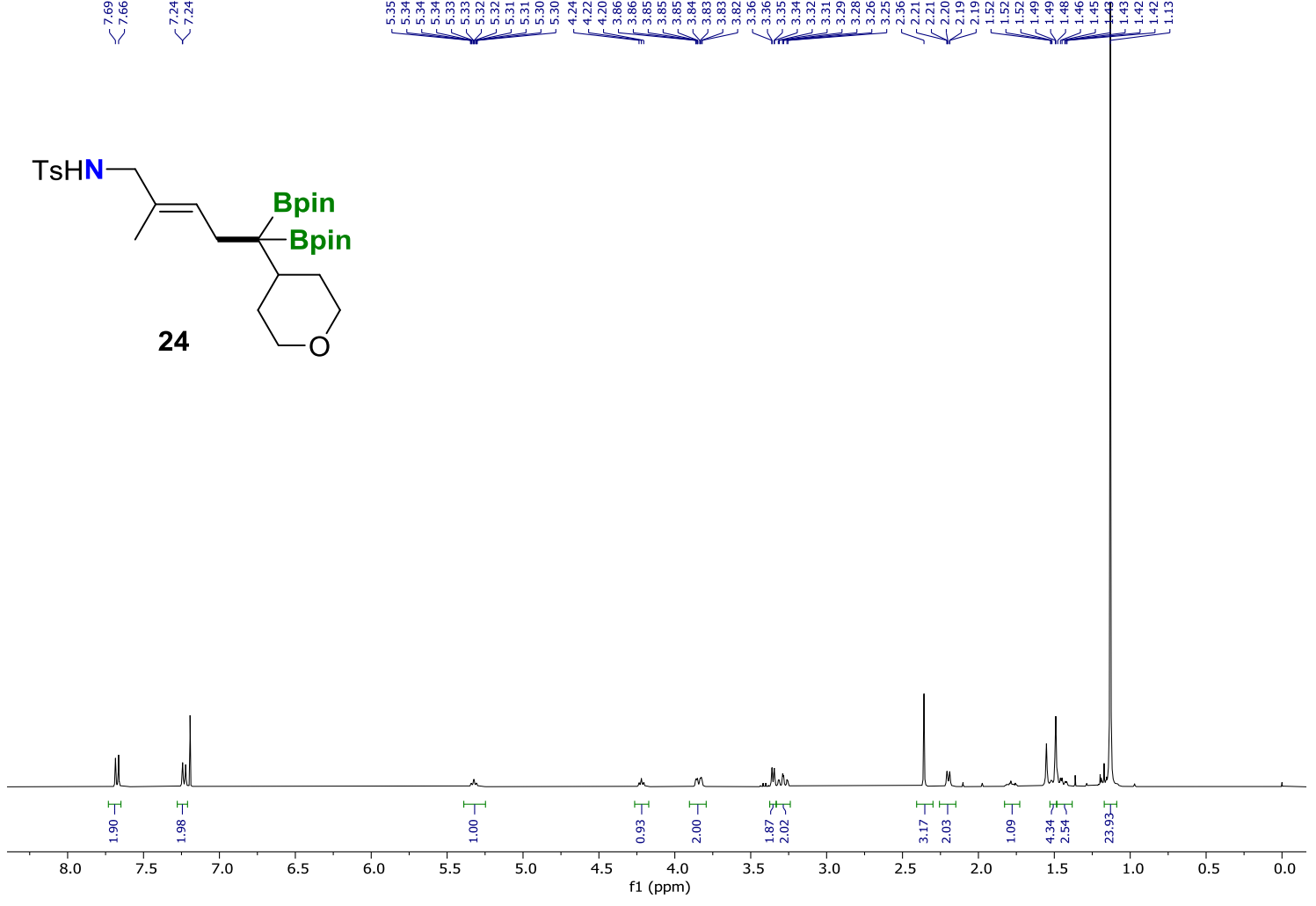




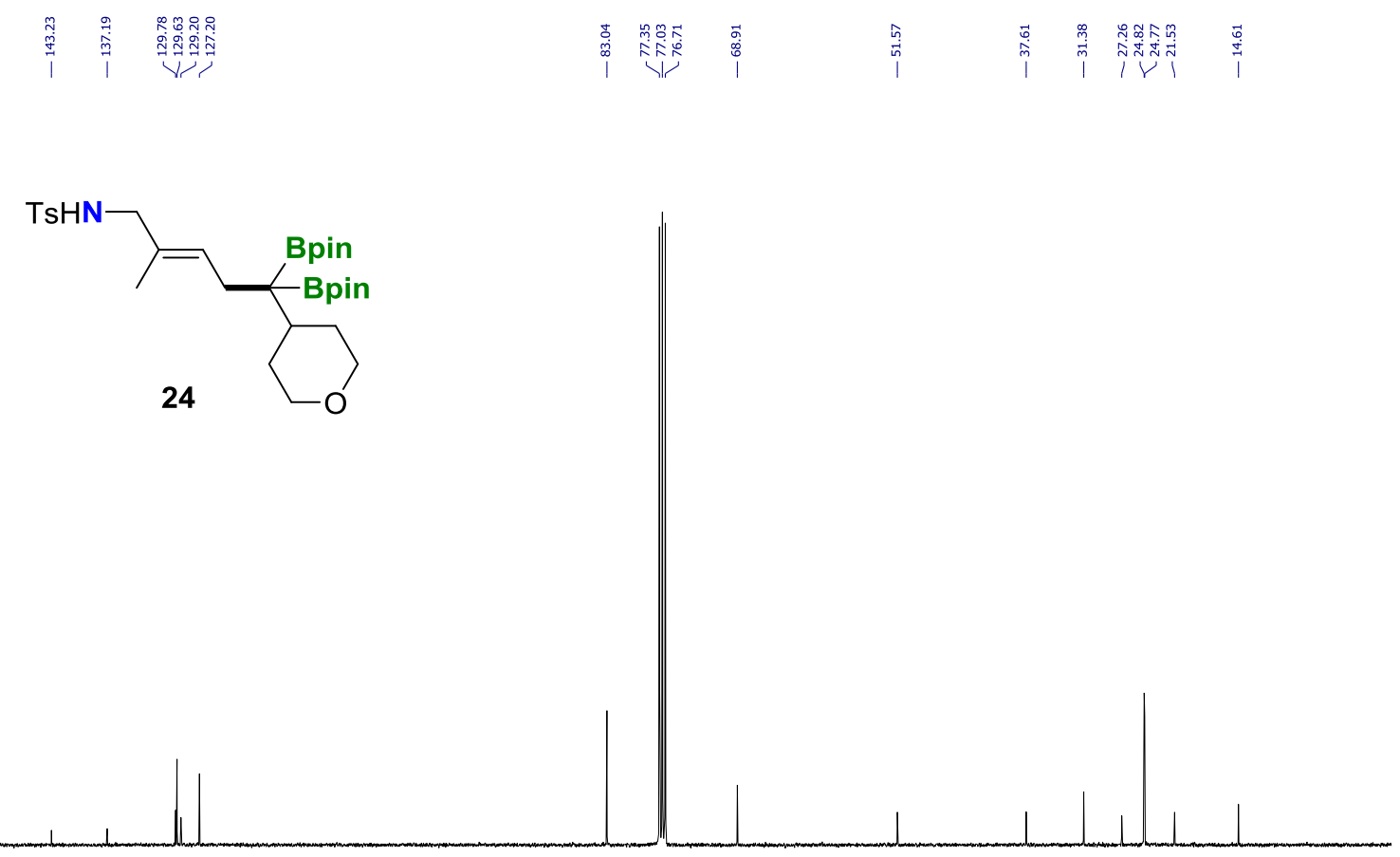

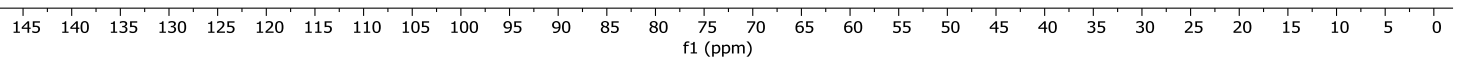

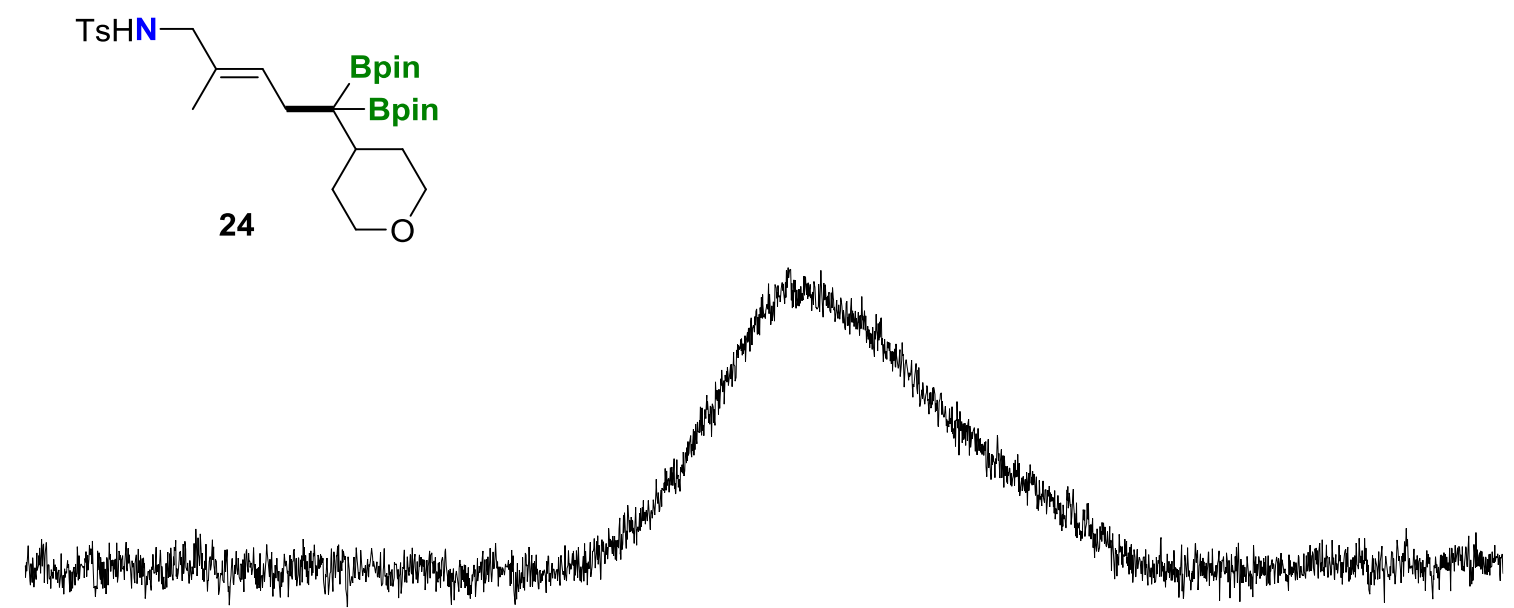

$\begin{array}{llllllllllllllllllllllllllllllllllllllll}52 & 51 & 50 & 49 & 48 & 47 & 46 & 45 & 44 & 43 & 42 & 41 & 40 & 39 & 38 & 37 & 36 & 35 & 34 & 3 & 32 & 31 & 30 & 29 & 18 & 27 & 26 & 25 & 14 & 13 & 22 & 21 & 1 & 1 & 18 & 17 & 16\end{array}$ 


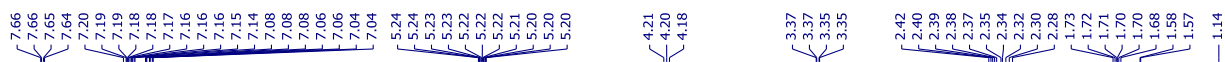

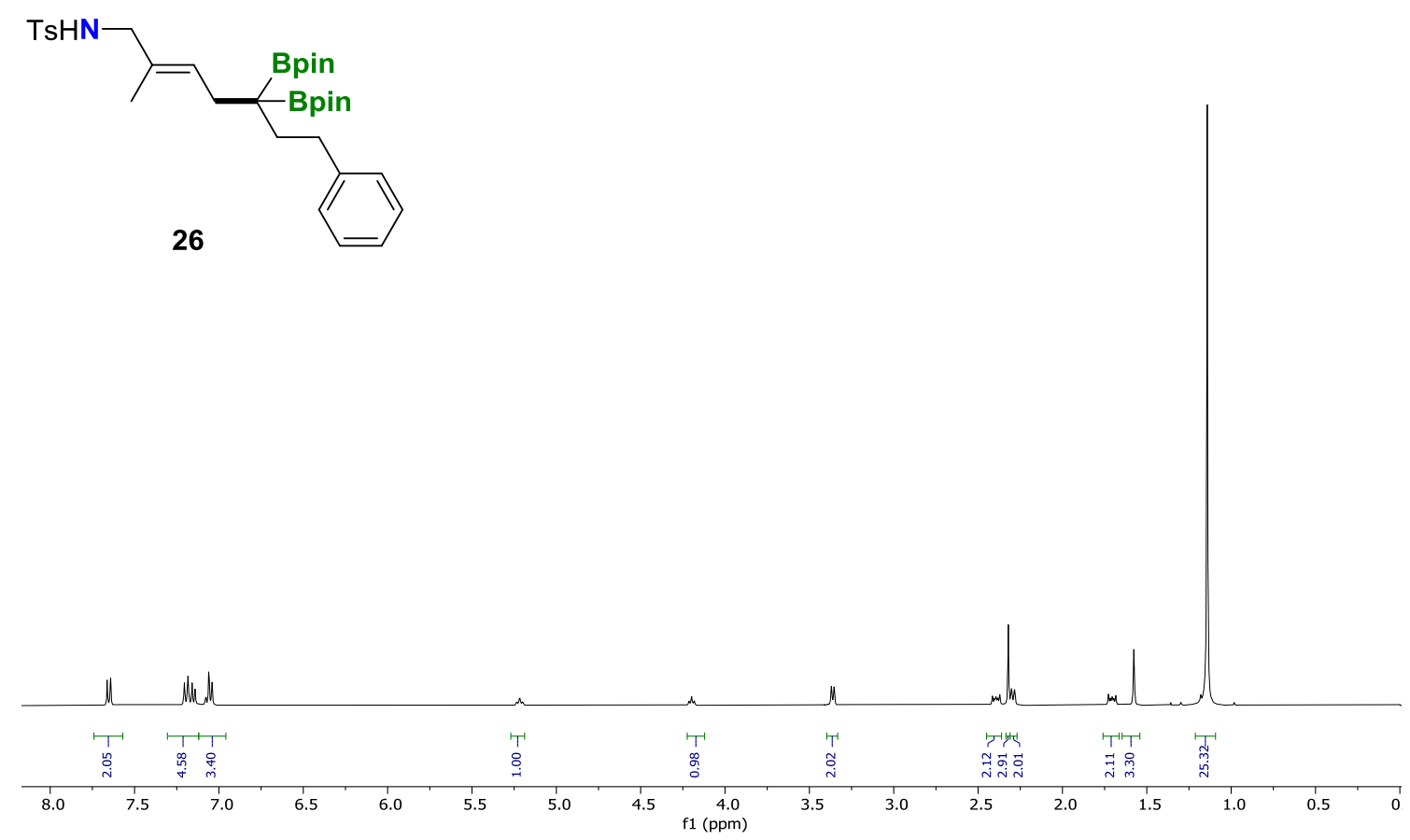

V)

i

ill
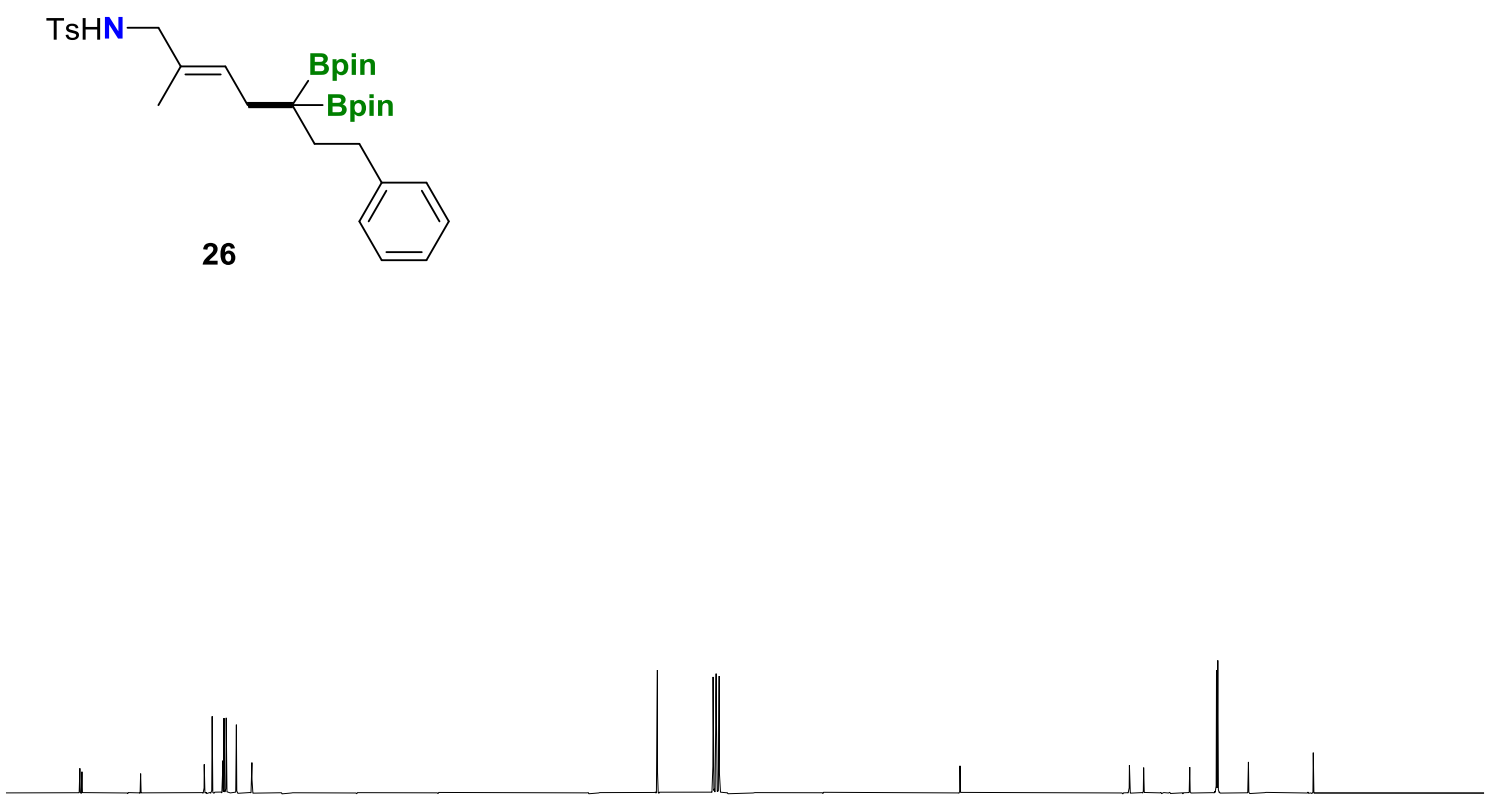

$\begin{array}{llllllllllllllllllllllllllllllllllllllllllllll}150 & 145 & 140 & 135 & 130 & 125 & 120 & 115 & 110 & 105 & 100 & 95 & 90 & 85 & 80 & 75 & 70 & 65 & 60 & 55 & 50 & 45 & 40 & 35 & 30 & 25 & 20 & 15 & 10 & 5 & 0\end{array}$ 

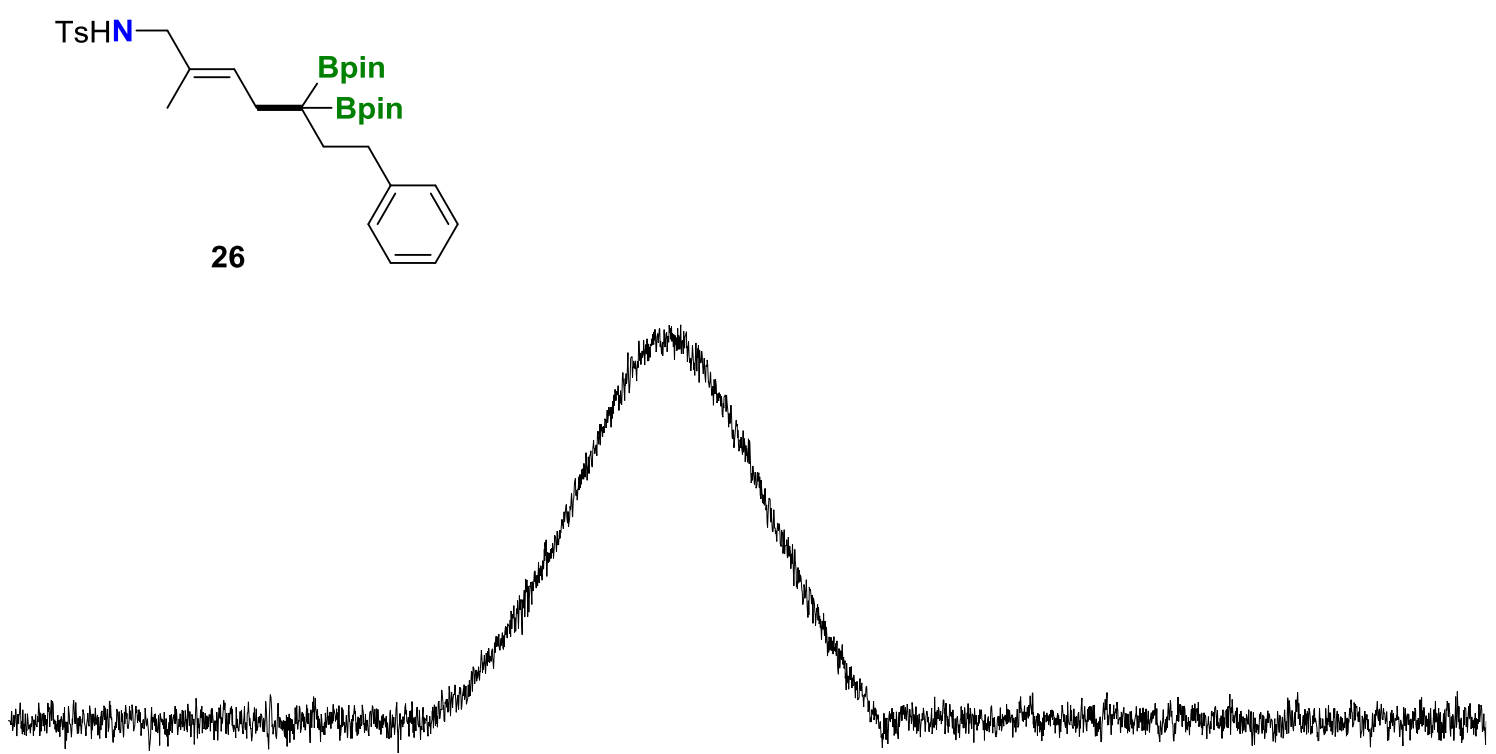

$\begin{array}{lllllllllllllllllllllllllllllllllllllllllllllll}51 & 50 & 49 & 48 & 47 & 46 & 45 & 44 & 43 & 42 & 41 & 40 & 39 & 38 & 37 & 36 & 35 & 34 & 33 & 32 & 31 & 30 & 29 & 28 & 27 & 26 & 25 & 24 & 23 & 22 & 21 & 20 & 19 & 18 & 17 & 16 & 15 & 14 & 13\end{array}$

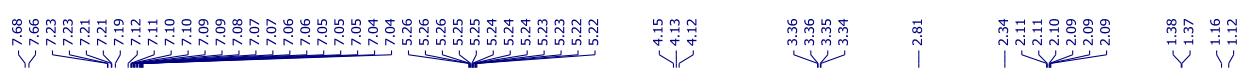
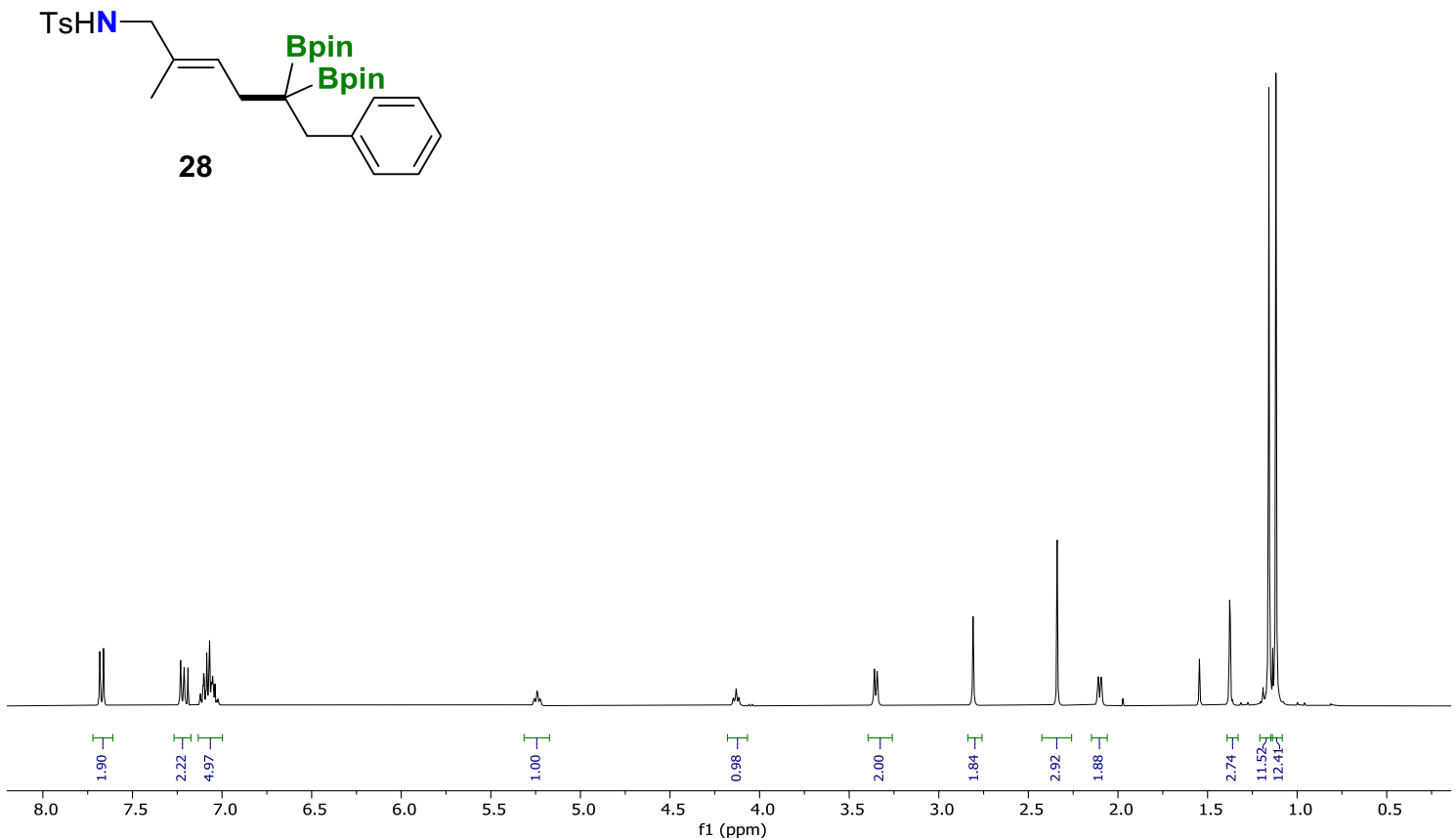

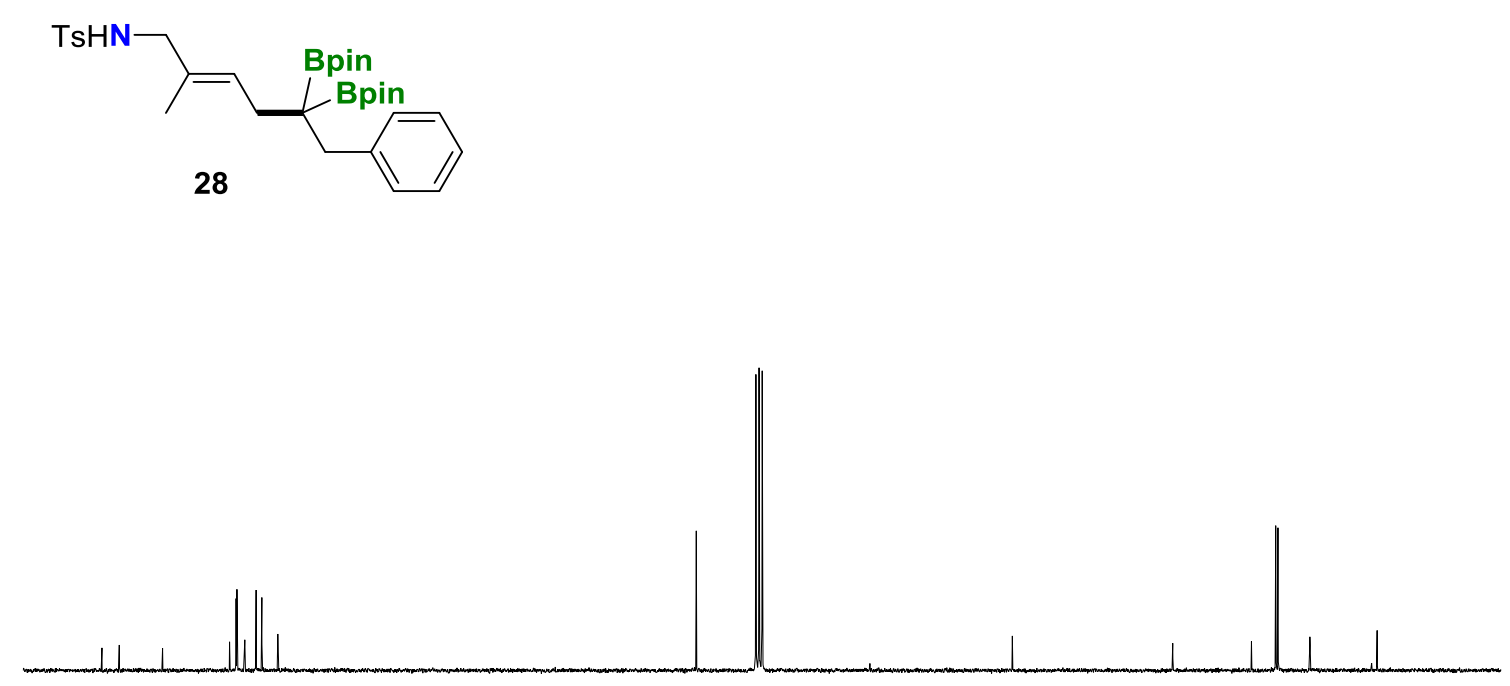

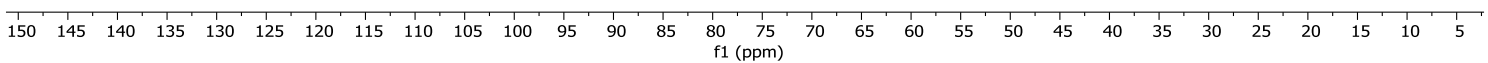
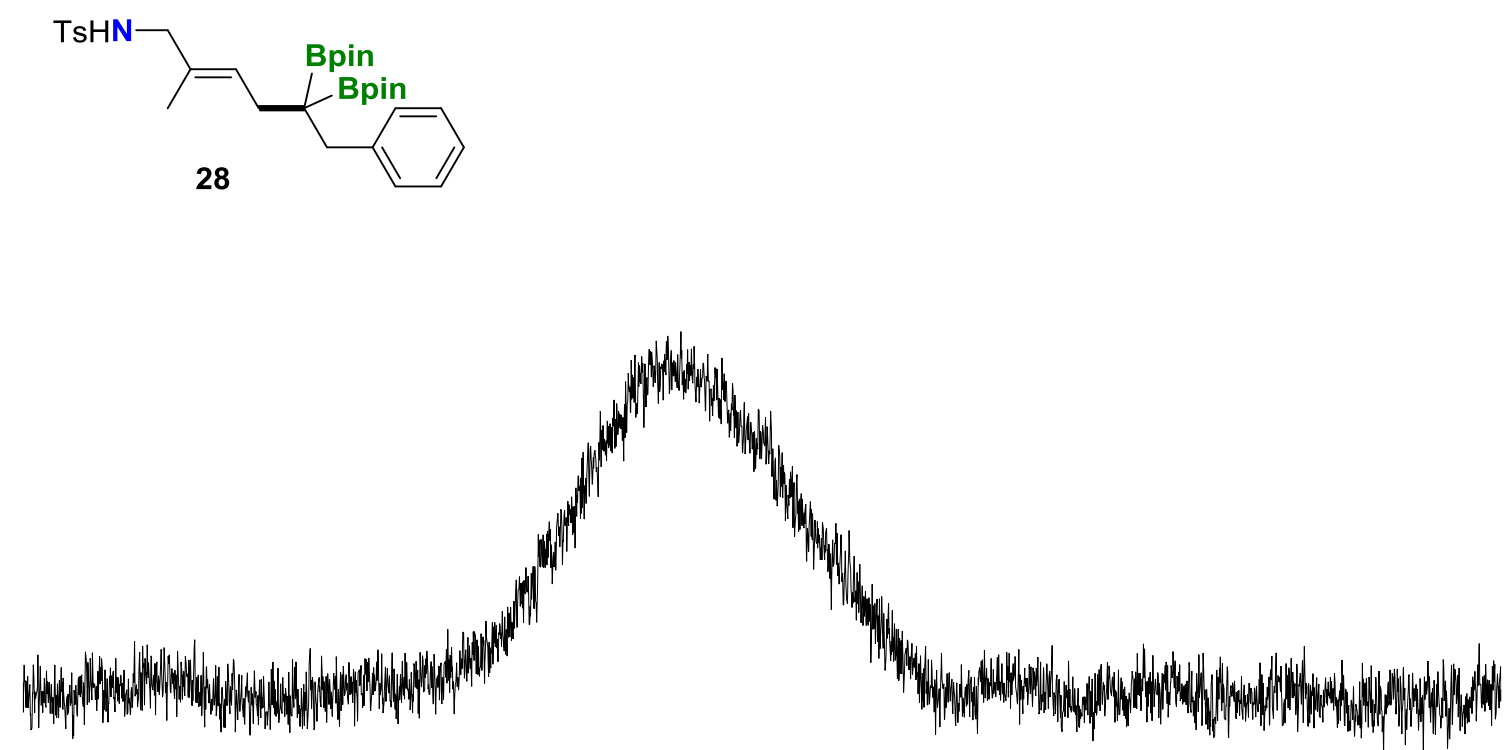

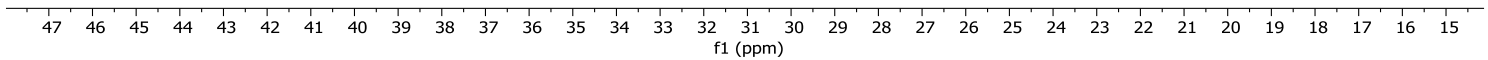



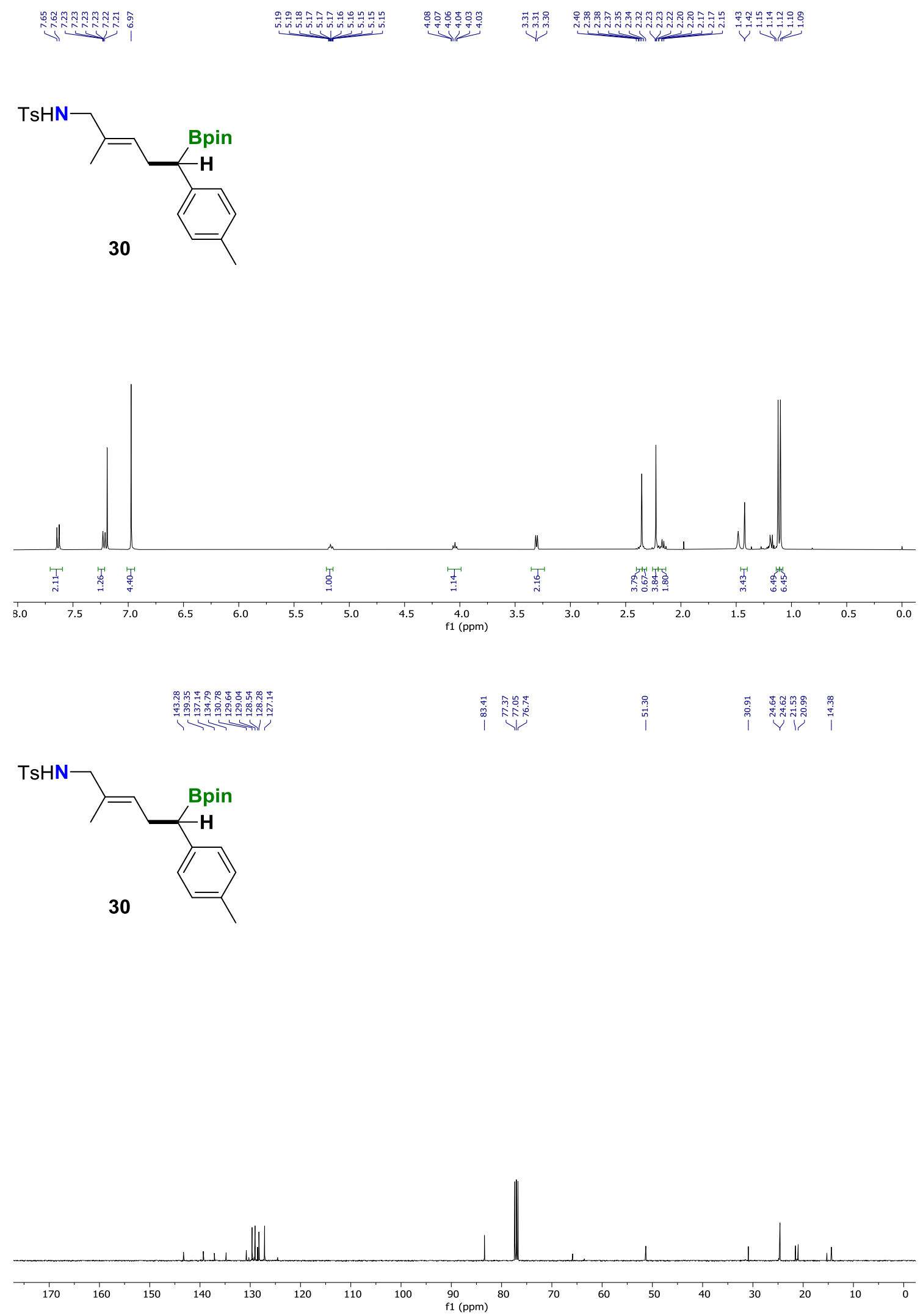

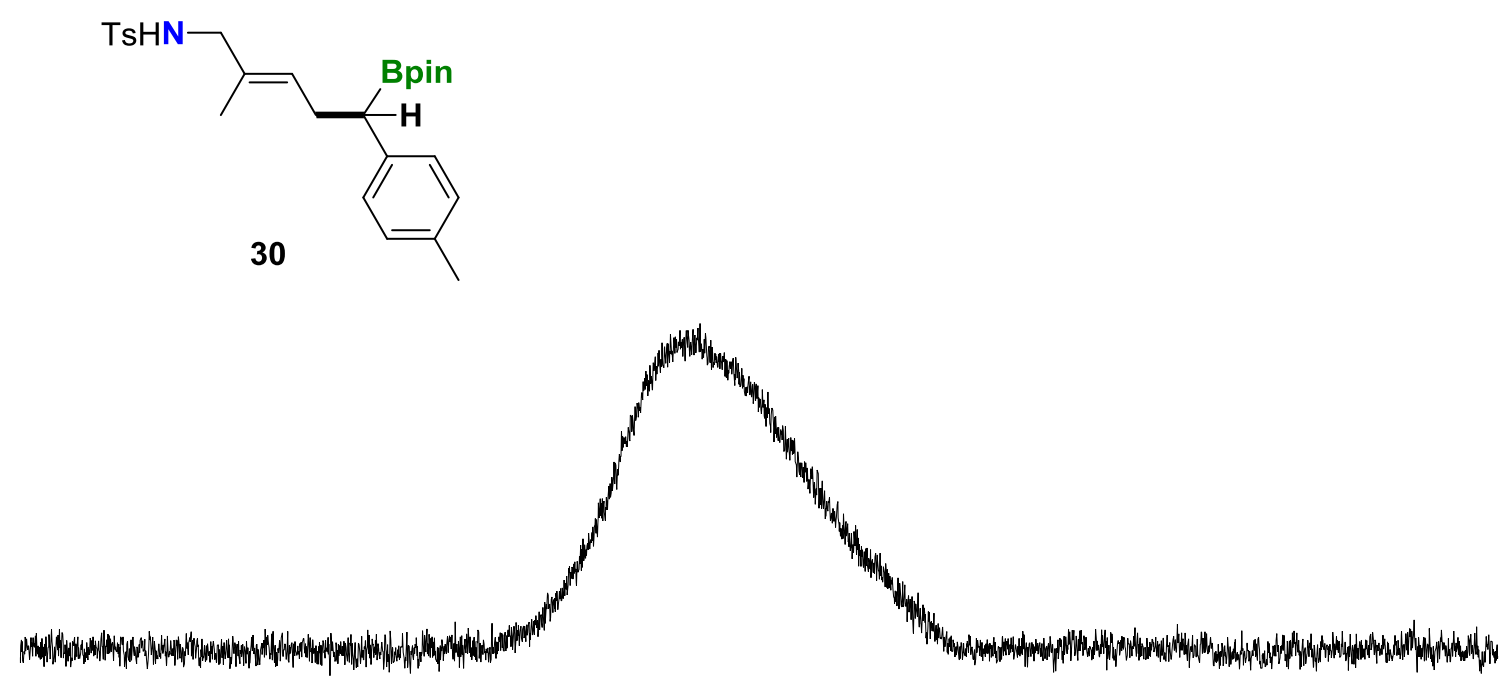

$\begin{array}{lllllllllllllllllllllllllllllllllllllllllllllllllllllll}49 & 48 & 47 & 46 & 45 & 44 & 43 & 42 & 41 & 40 & 39 & 38 & 37 & 36 & 35 & 34 & 33 & 32 & 31 & 30 & 29 & 28 & 27 & 26 & 25 & 24 & 23 & 22 & 21 & 20 & 19 & 18 & 17 & 16 & 15 & 14 & 13 & 12 & 1\end{array}$

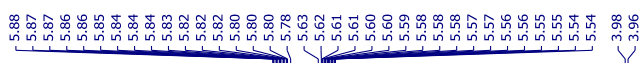

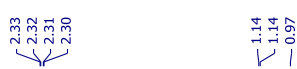

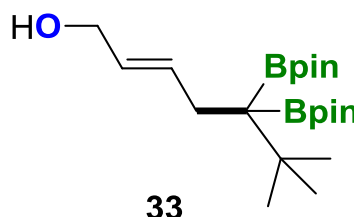

33

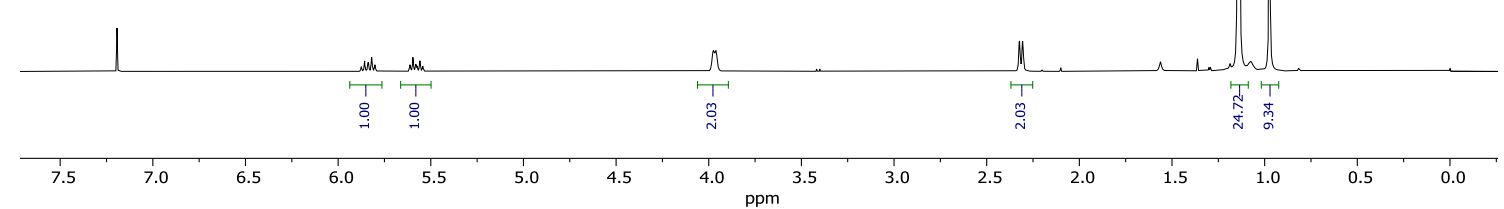



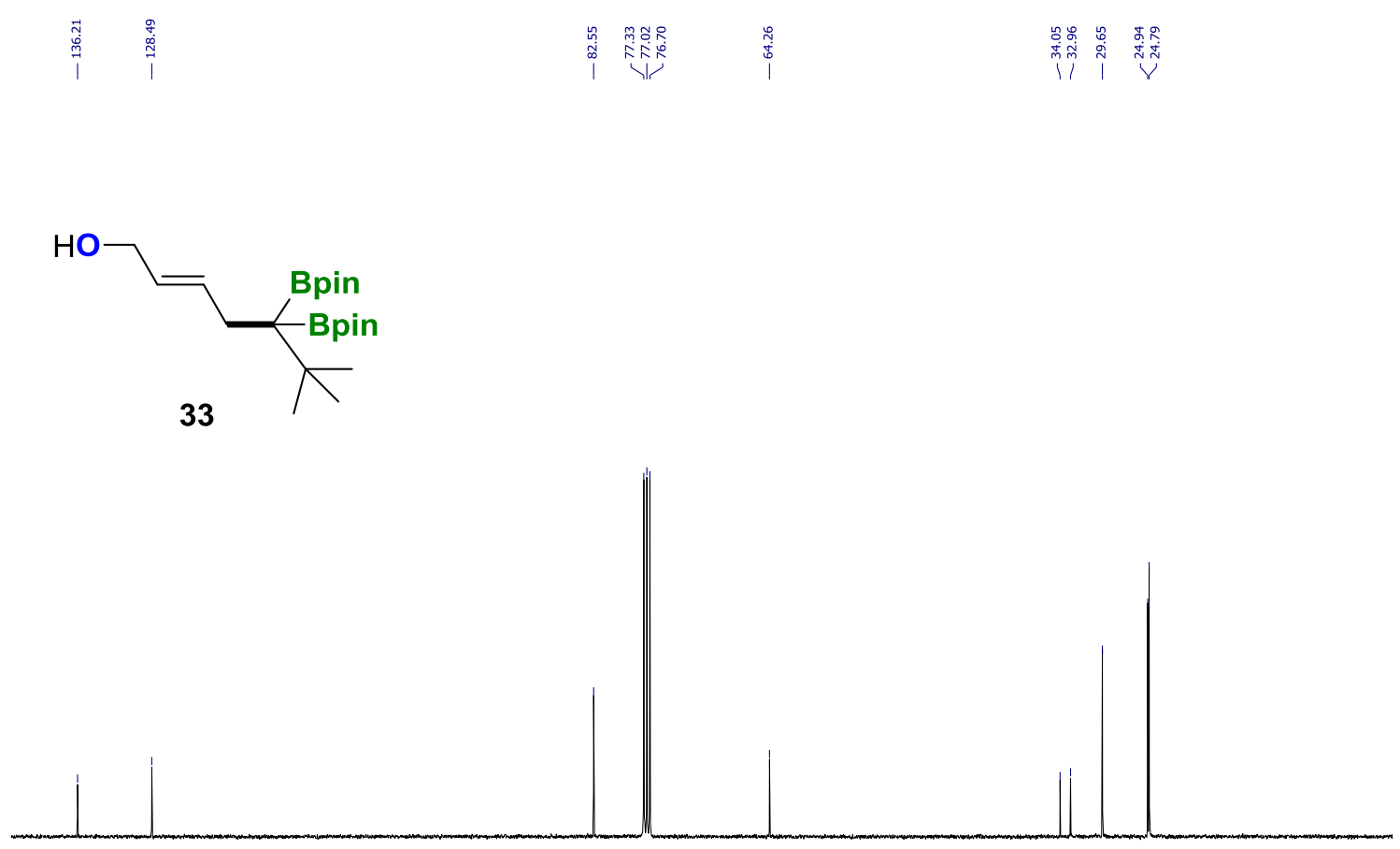

$\begin{array}{lllllllllllllllllllllllllllllllllllllllllll}140 & 135 & 130 & 125 & 120 & 115 & 110 & 105 & 100 & 95 & 90 & 85 & 80 & 75 & 70 & 65 & 60 & 55 & 50 & 45 & 40 & 35 & 30 & 25 & 20 & 15 & 10 & 5 & 0\end{array}$
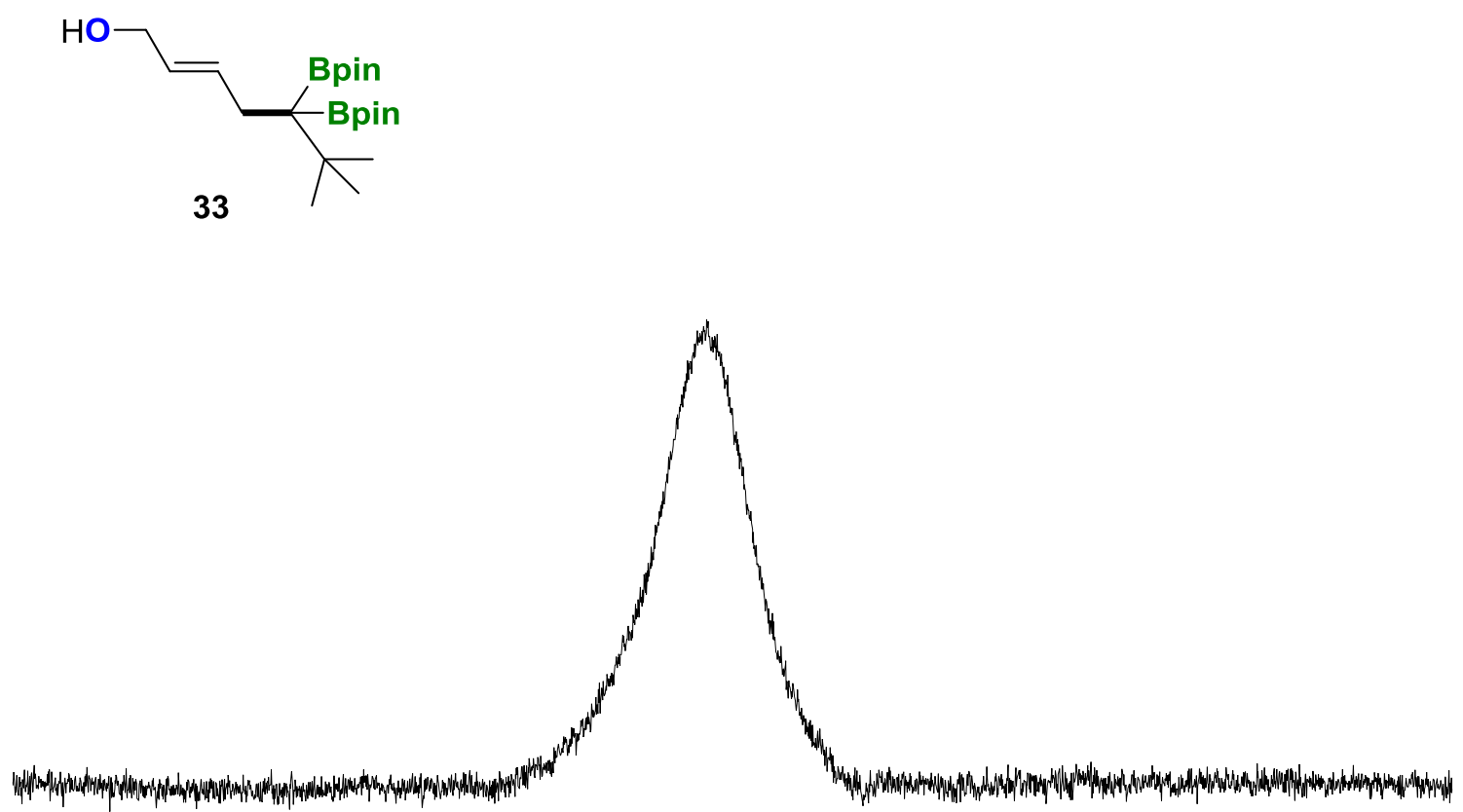

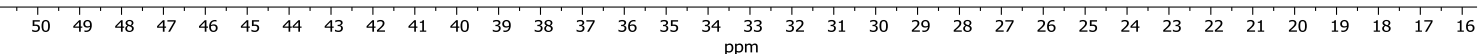




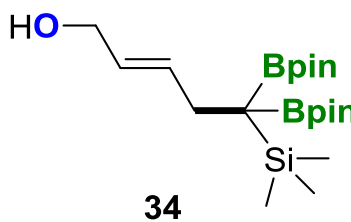

34

Si-
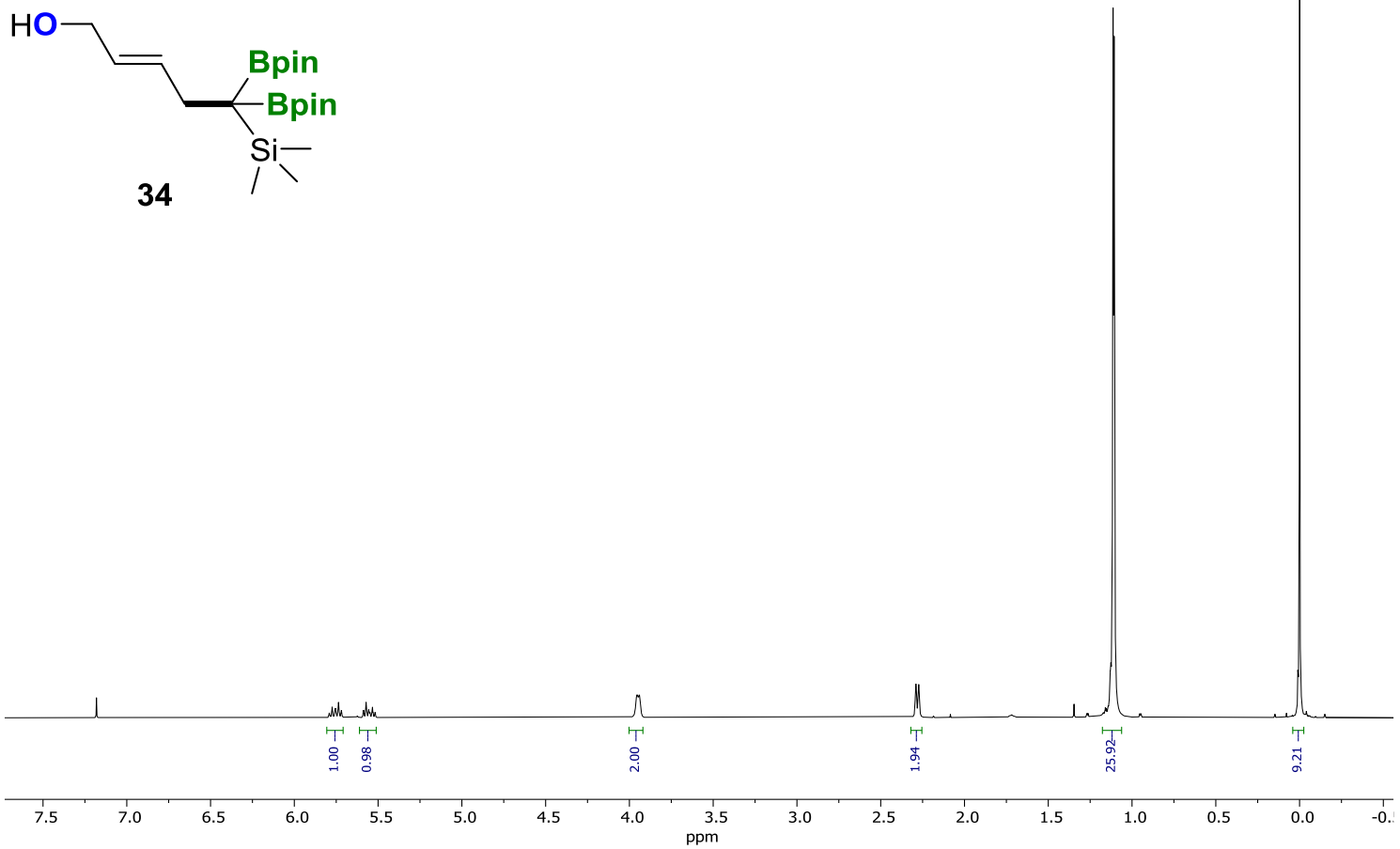

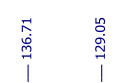

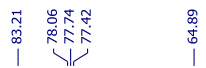

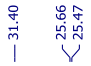

$\vec{b}$
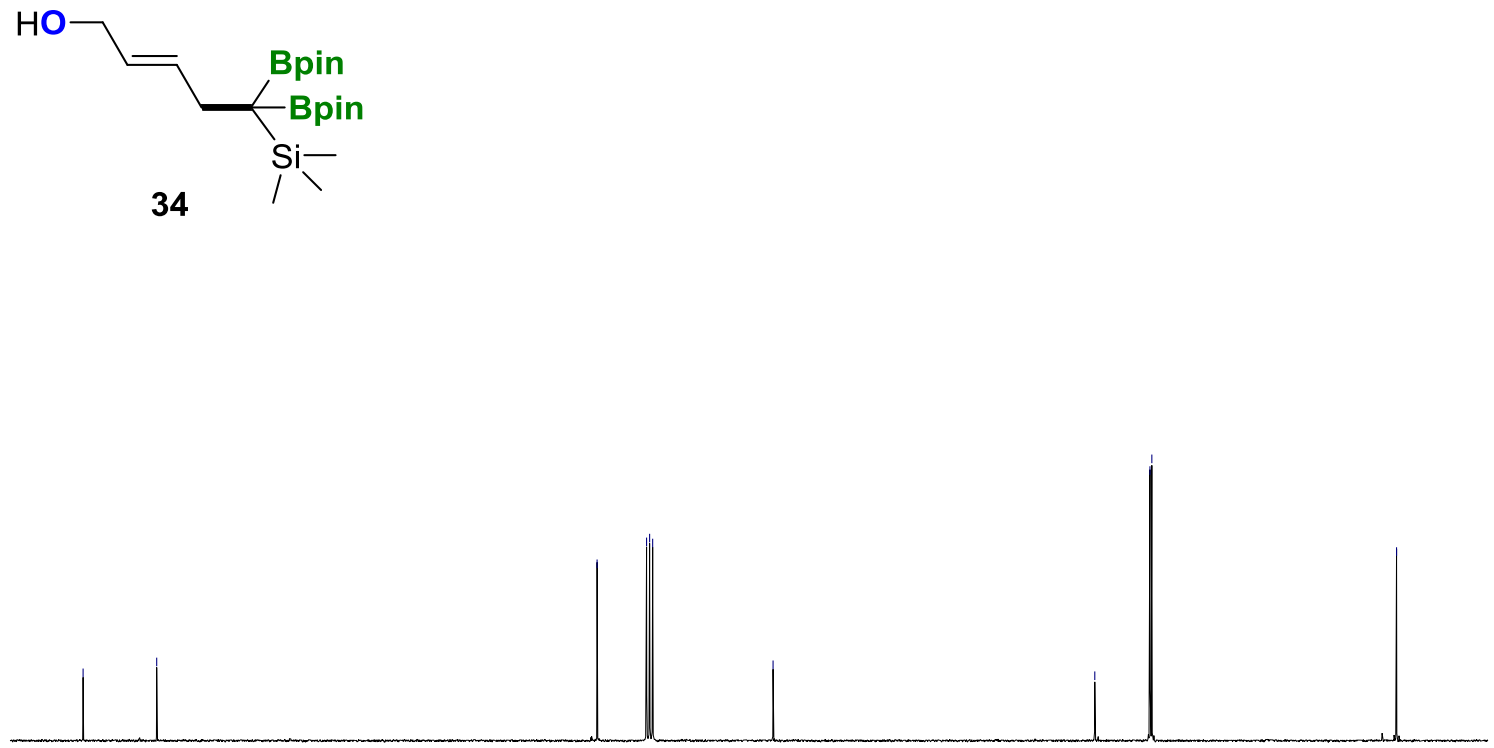

$\begin{array}{lllllllllllllllllllllllllllllllllllllllllllllllll}140 & 135 & 130 & 125 & 120 & 115 & 110 & 105 & 100 & 95 & 90 & 85 & 80 & 75 & 70 & 65 & 60 & 55 & 50 & 45 & 40 & 35 & 30 & 25 & 20 & 15 & 10 & 5 & 0 & -5\end{array}$ 


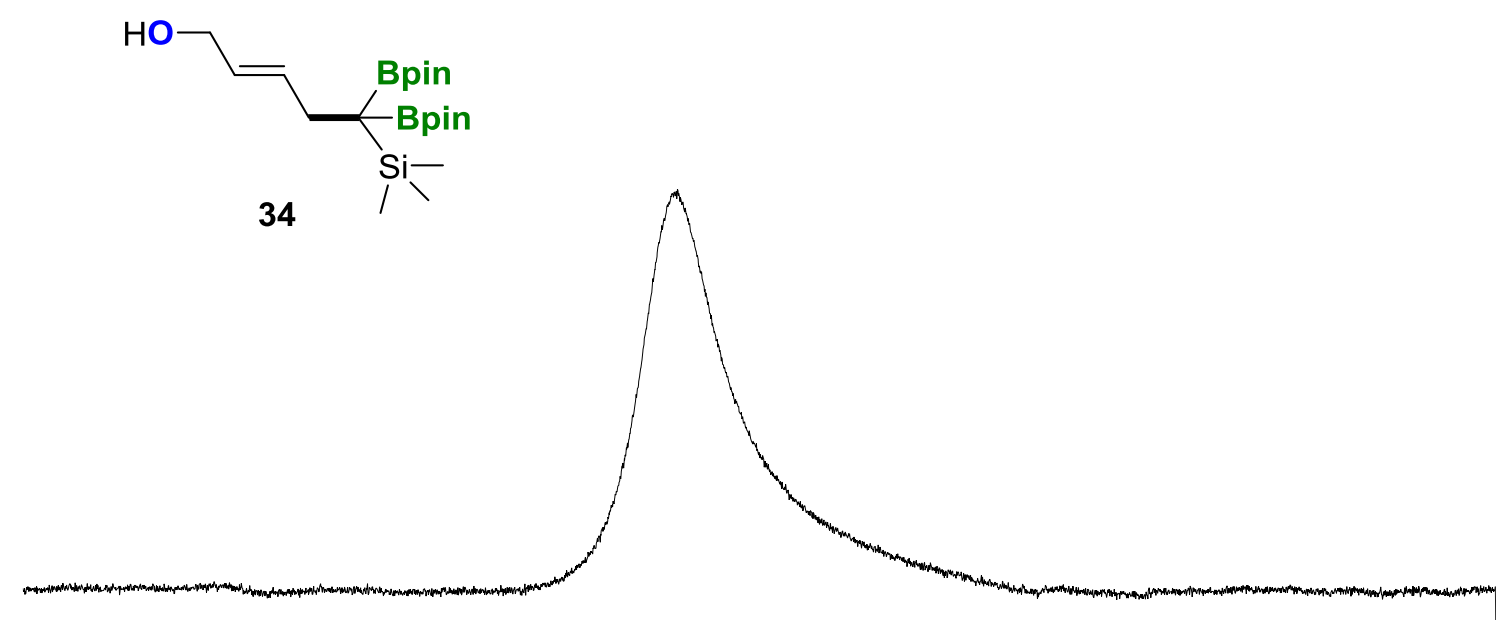

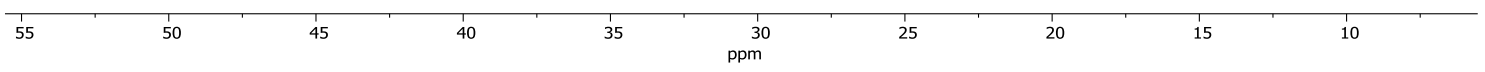
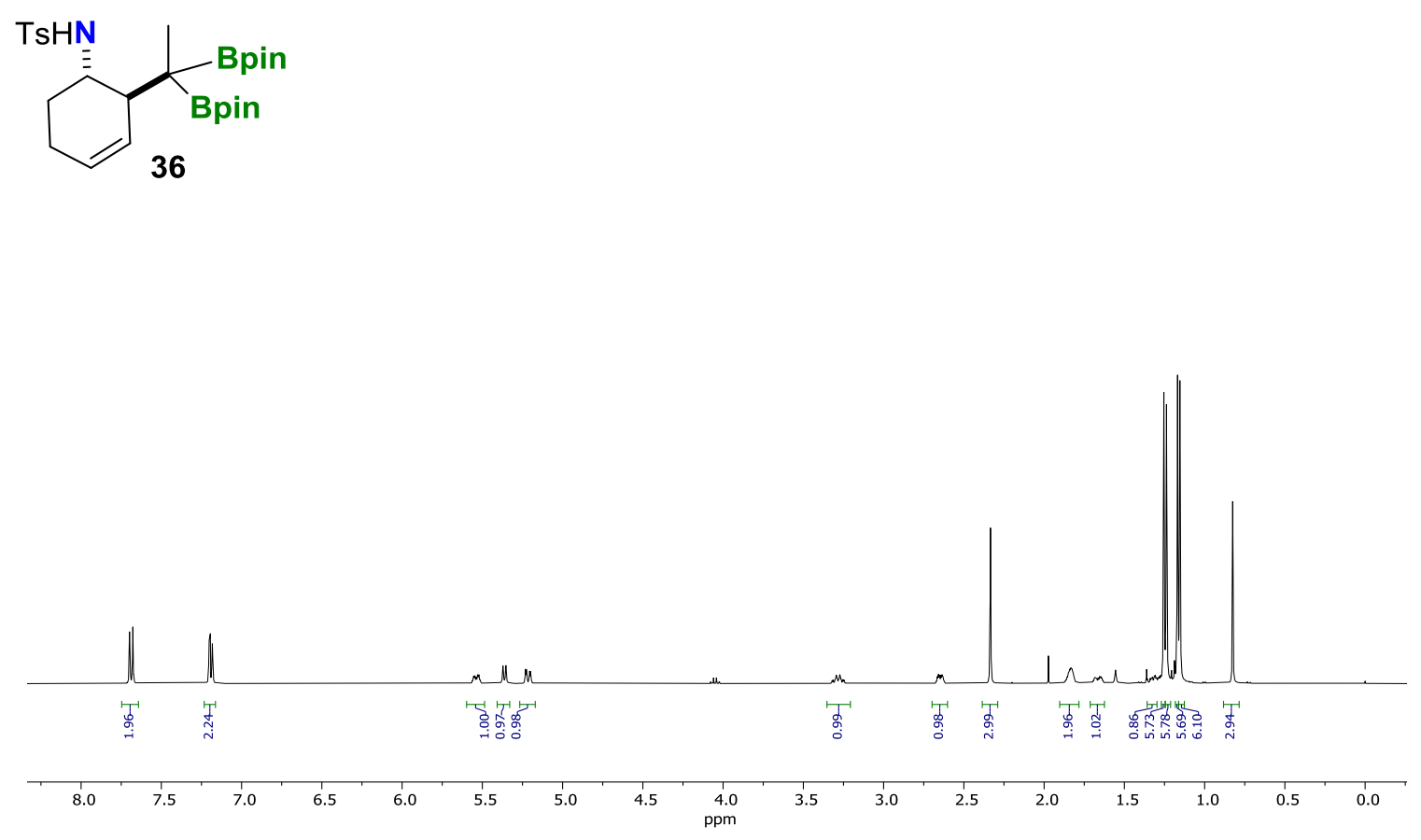

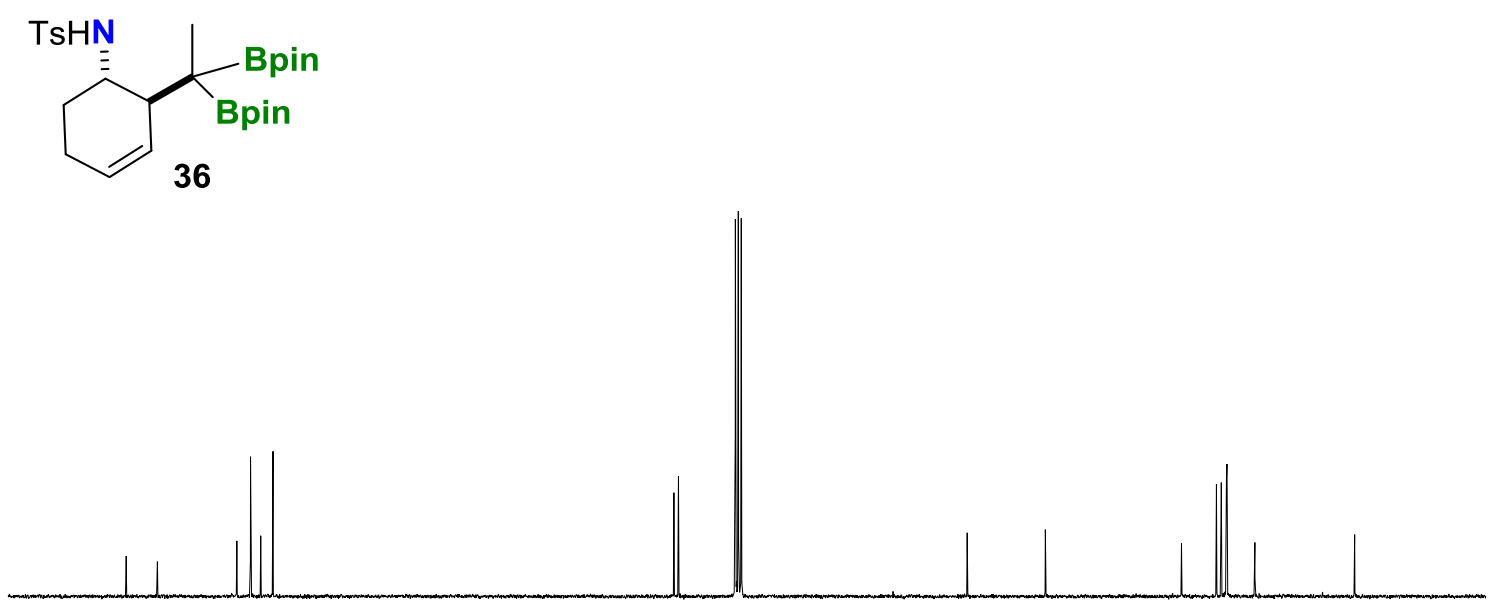

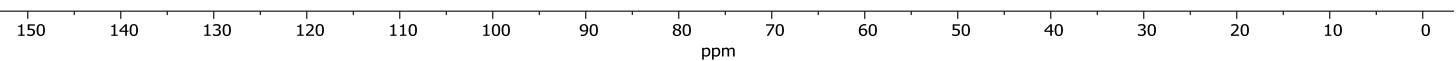
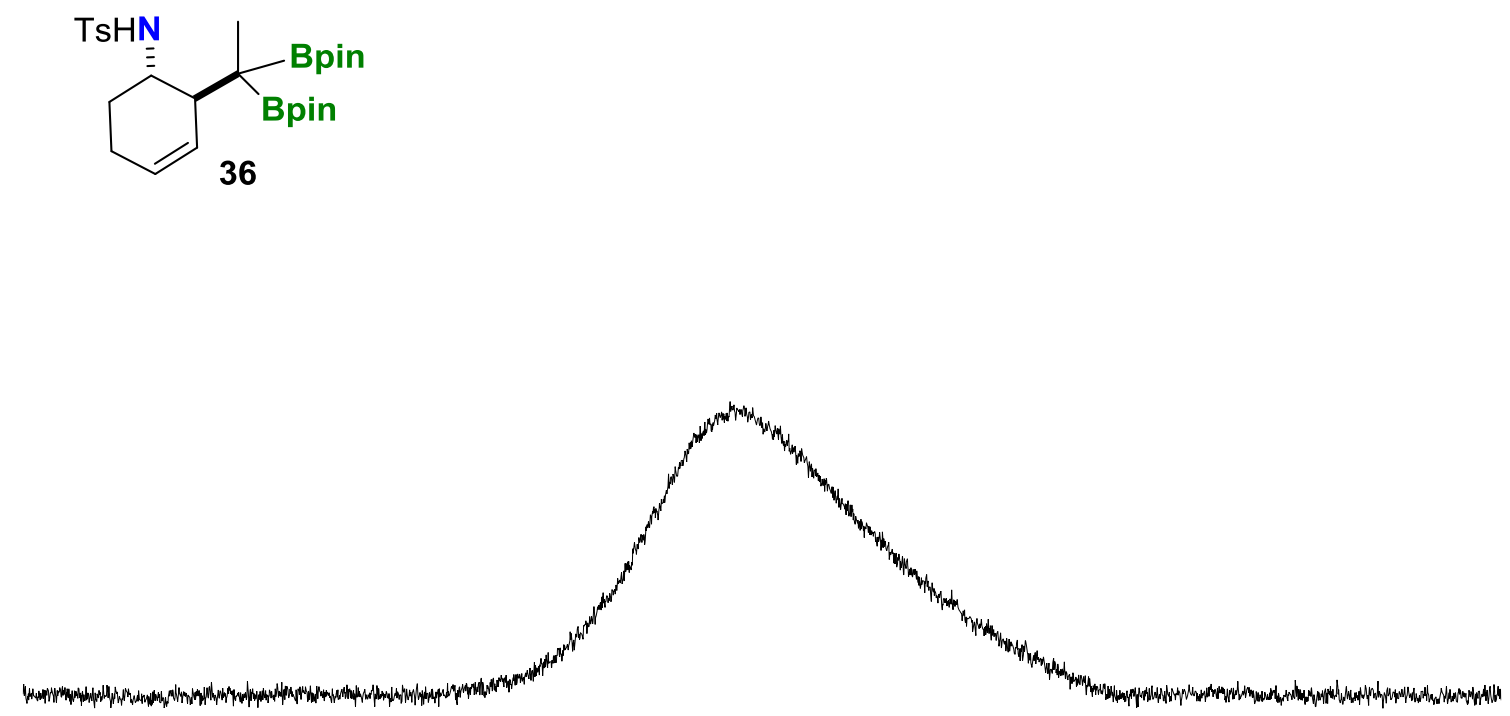

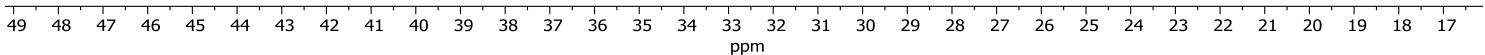



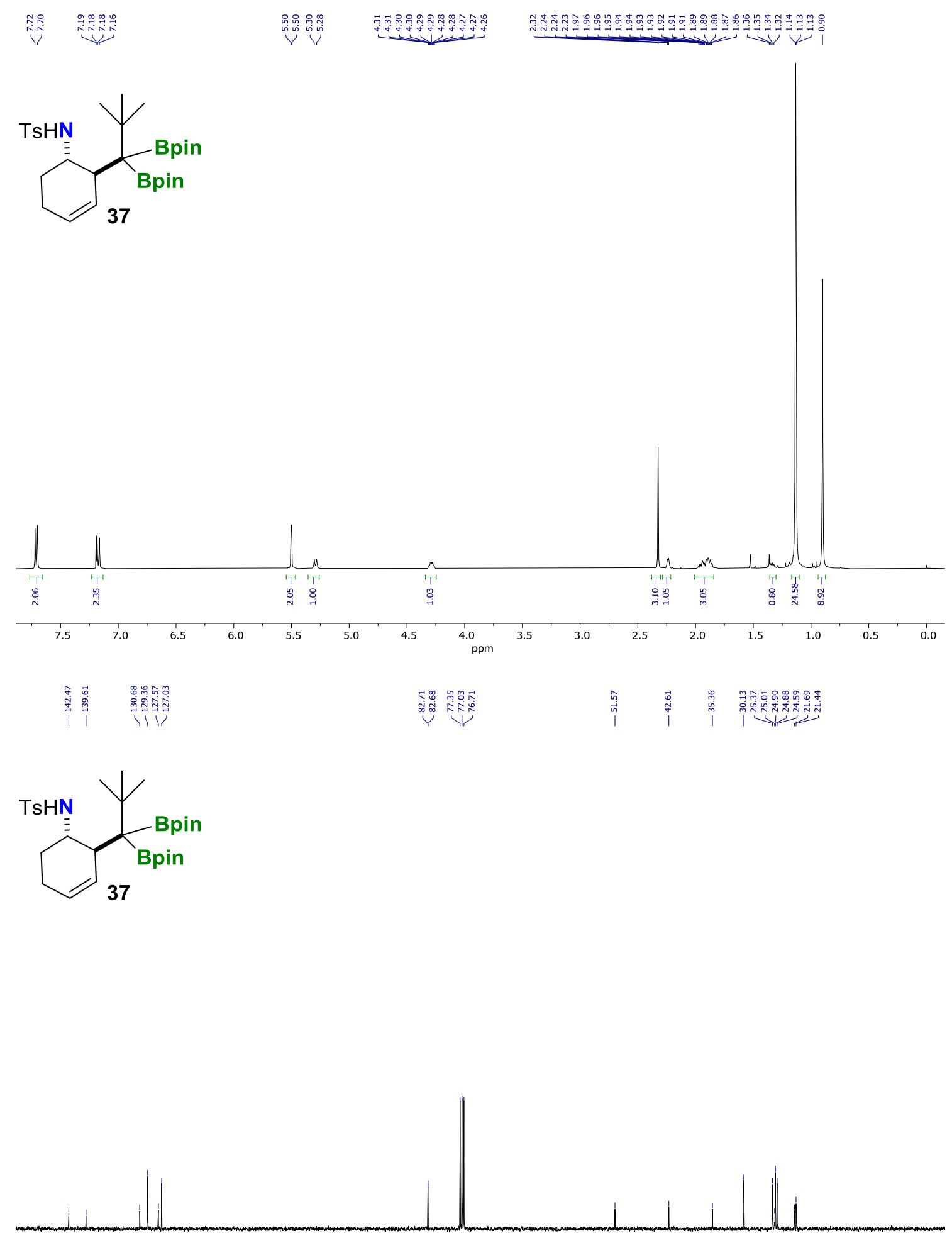

$\begin{array}{llllllllllllllllllllllllllllllllllllllllllllllllllllll}150 & 145 & 140 & 135 & 130 & 125 & 120 & 115 & 110 & 105 & 10 & 95 & 90 & 85 & 80 & 75 & 70 & 65 & 60 & 55 & 50 & 45 & 40 & 35 & 30 & 25 & 20 & 15 & 10 & 5 & 0\end{array}$ 

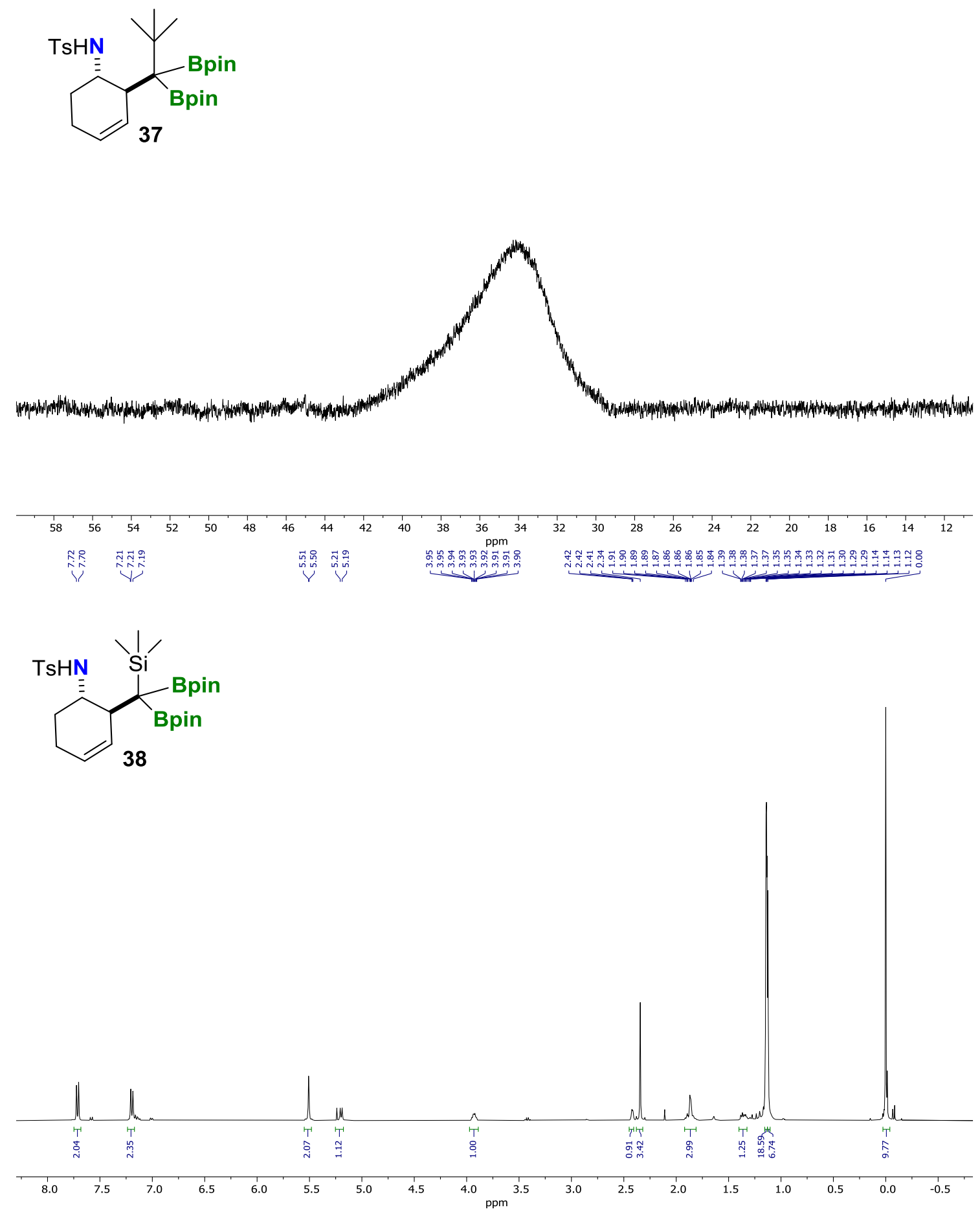


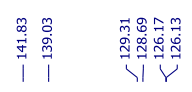

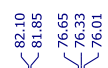

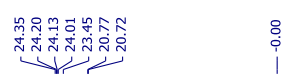
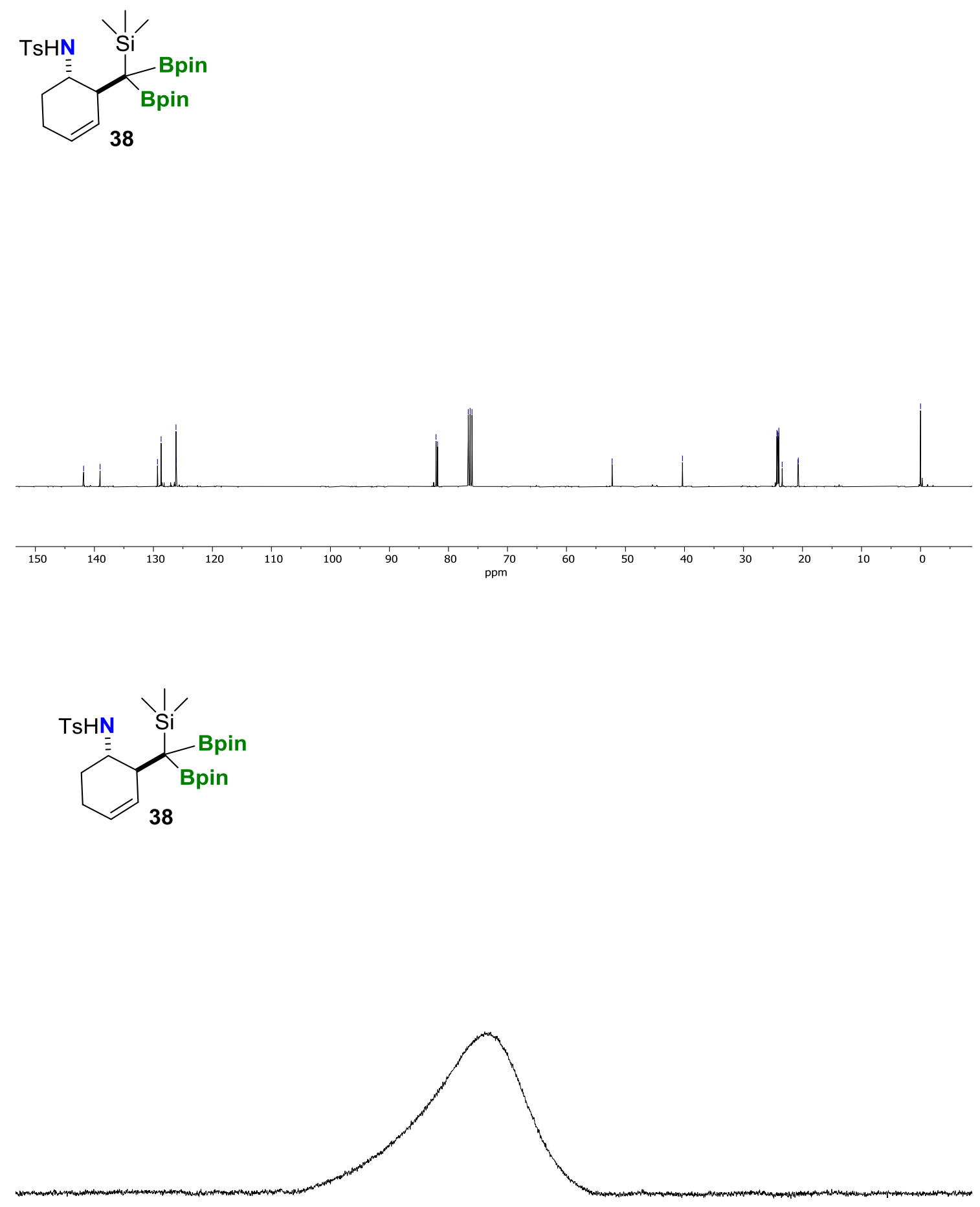

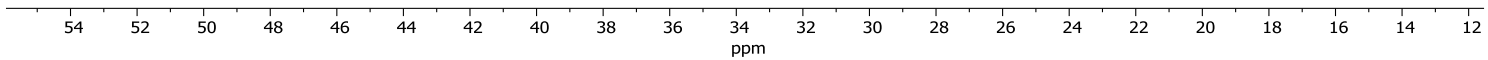


<smiles>[NH3+][C@@H]1CCC=C[C@H]1C(Br)(Cc1ccccc1)C1CCOCC1</smiles>

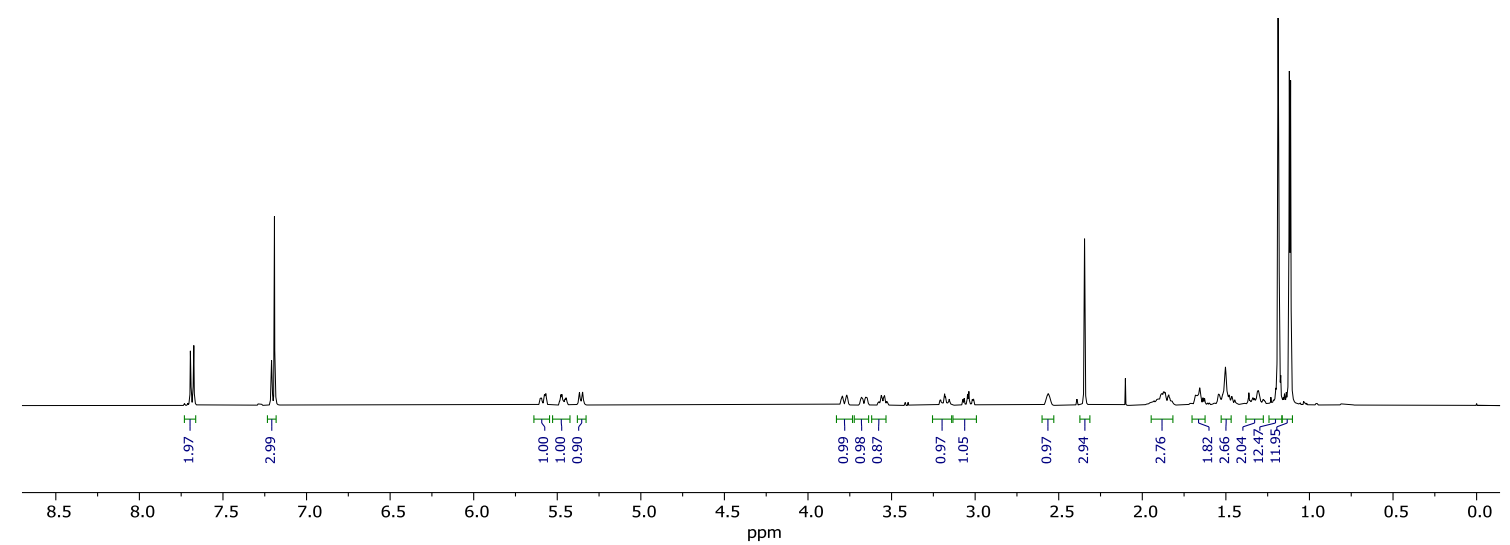<smiles>[NH3+][C@@H]1CCC=C[C@H]1C(Br)(Cc1ccccc1)C1CCOCC1</smiles>

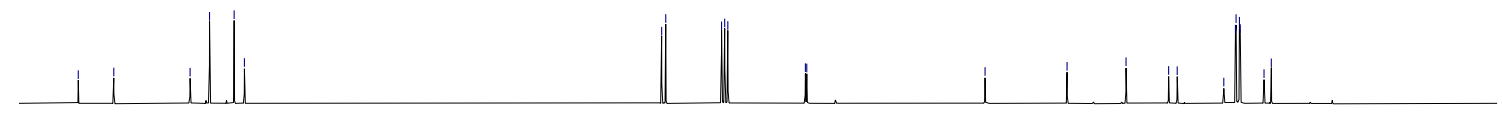

$\begin{array}{llllllllllllllllllllllllllllllllllllllllllllll}145 & 140 & 135 & 130 & 125 & 120 & 115 & 110 & 105 & 100 & 95 & 90 & 85 & 80 & 75 & 70 & 65 & 60 & 55 & 50 & 45 & 40 & 35 & 30 & 25 & 20 & 15 & 10 & 5 & 0\end{array}$ 


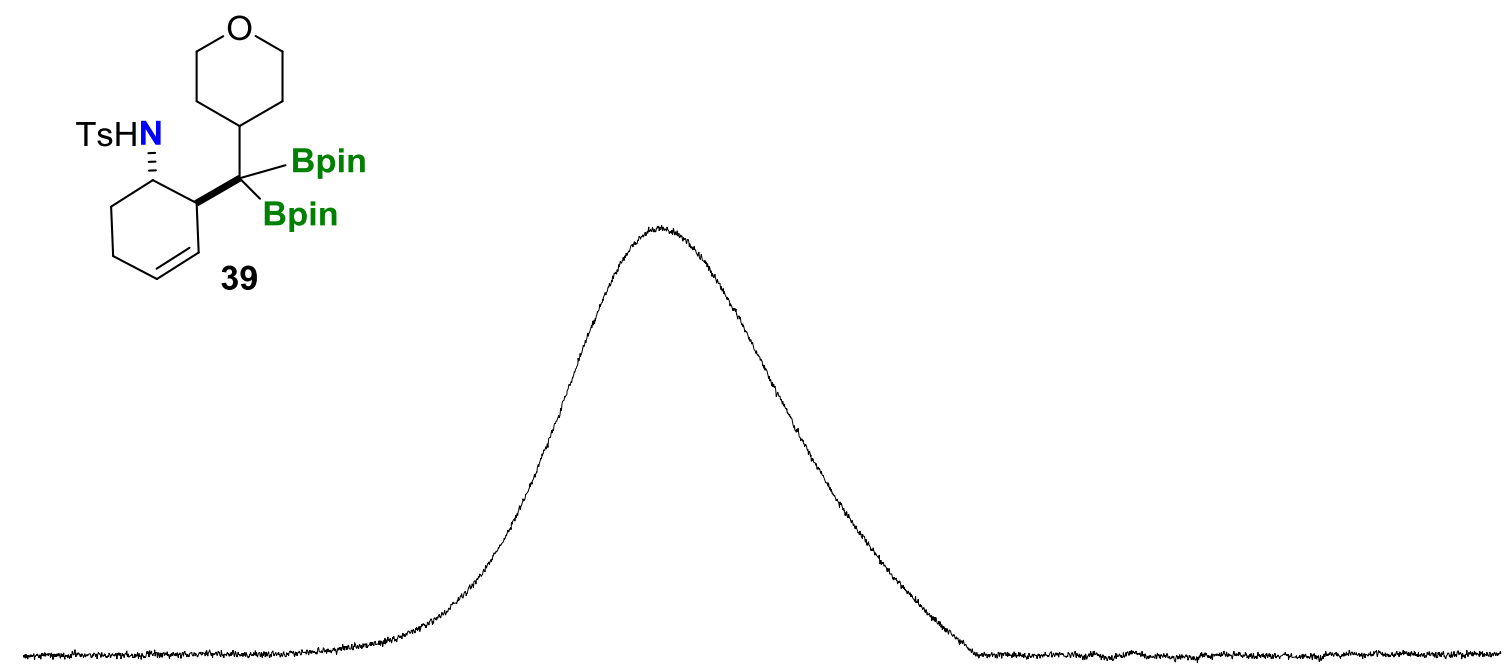

$\begin{array}{lllllllllllllllllllllllllllllllllllllllllll}0 & 49 & 48 & 47 & 46 & 45 & 44 & 43 & 42 & 41 & 40 & 39 & 38 & 37 & 36 & 35 & 34 & 33 & 32 & 31 & 30 & 29 & 28 & 17 & 26 & 15 & 24 & 13 & 22 & 12 & 20 & 19 & 18 & 1 & 16 & 15 & 14 & 13 & 12 & 11 & 10\end{array}$<smiles>[NH3+][C@H]1CCC=C[C@H]1C(Br)(Cc1ccccc1)c1ccccc1</smiles>

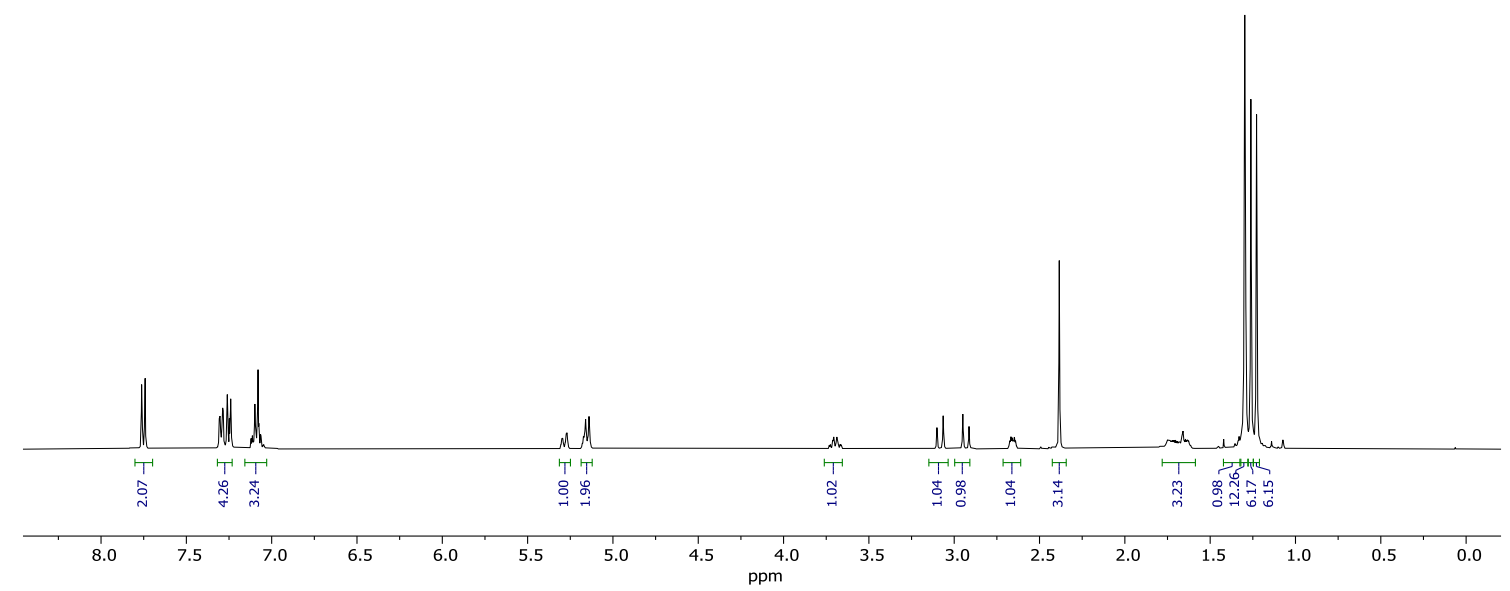


<smiles>[NH3+][C@H]1CCC=C[C@H]1C(Br)(Br)c1ccccc1</smiles>

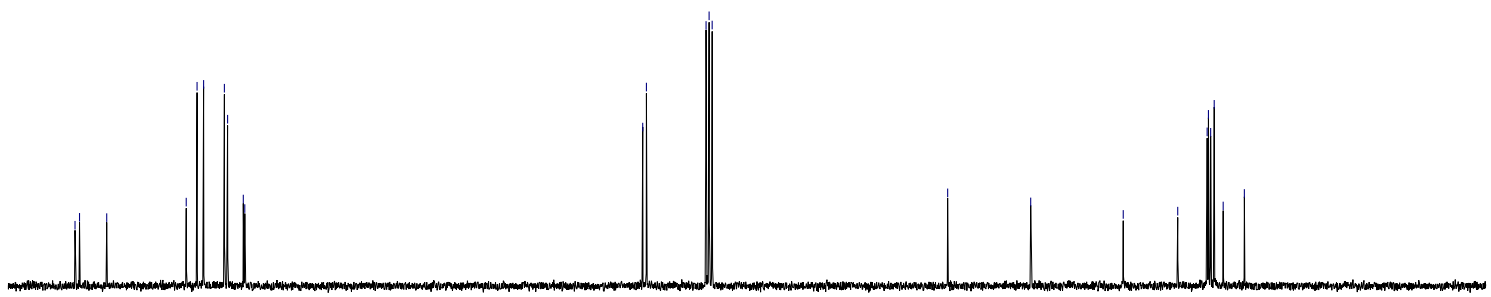

$\begin{array}{lllllllllllllllllllllllllllllllllllllllllll}145 & 140 & 135 & 130 & 125 & 120 & 115 & 110 & 105 & 100 & 95 & 90 & 85 & 80 & 75 & 70 & 65 & 60 & 55 & 50 & 45 & 40 & 35 & 30 & 25 & 20 & 15 & 10 & 5 & 0\end{array}$<smiles>[NH3+]C1CCC=CC1C(Br)(Br)c1ccccc1</smiles>

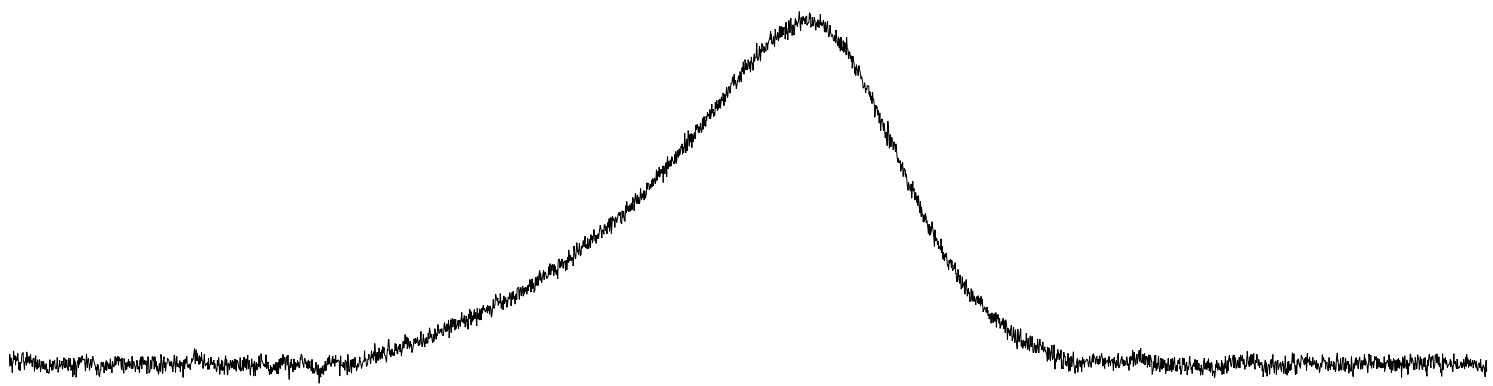

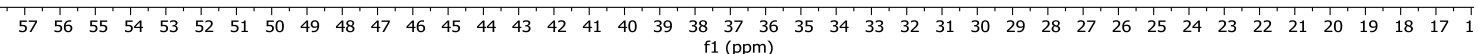


<smiles>Cc1ccc(C(CBr)C2C=CCC[C@H]2[AlH2])cc1</smiles>
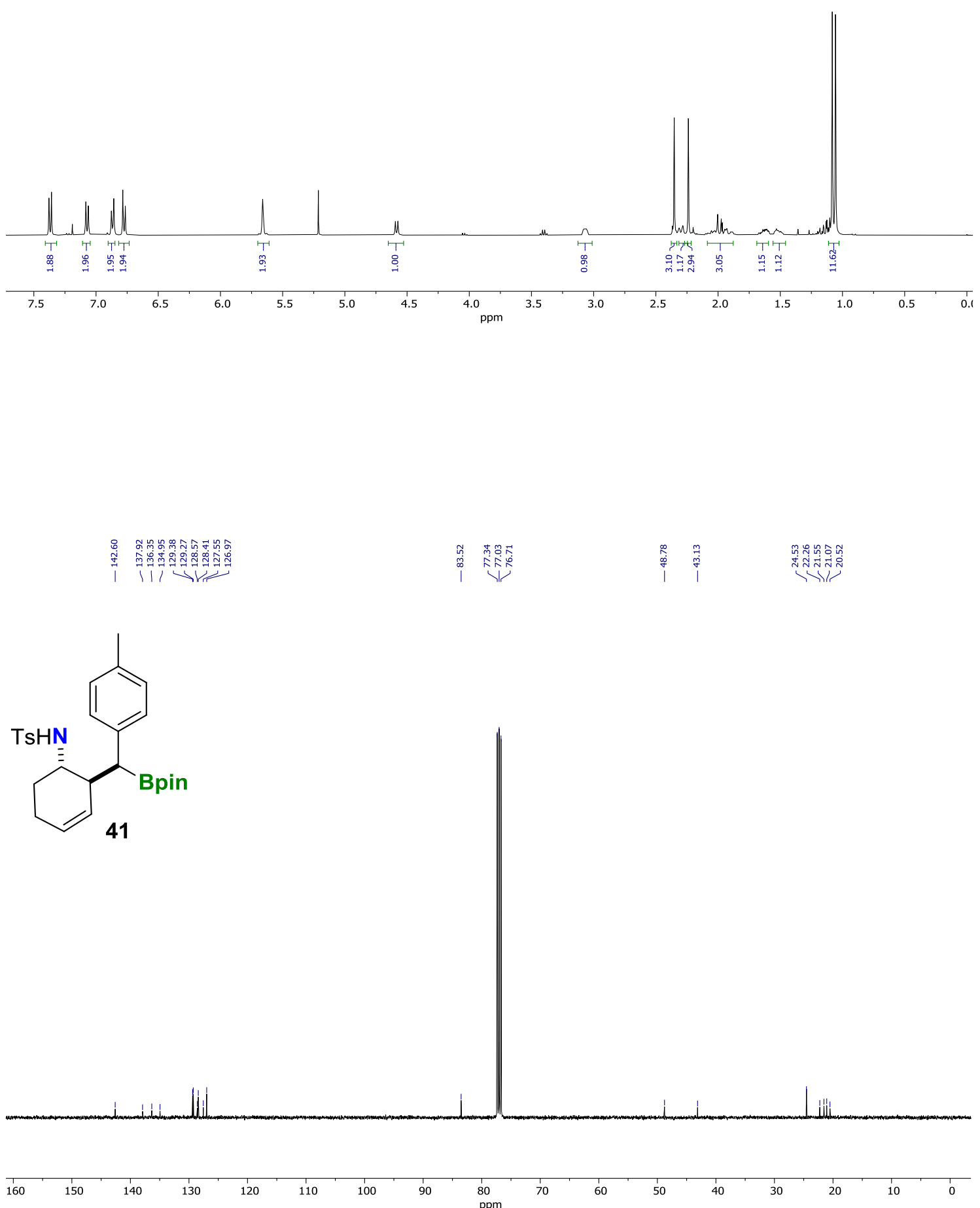


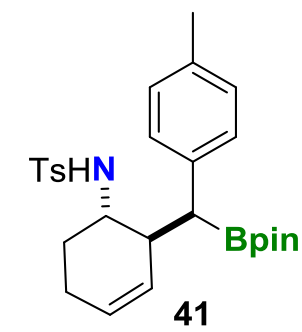

$\begin{array}{lllllllllllllllllllllllllllllllllllllllllllllll}54 & 53 & 52 & 51 & 50 & 49 & 48 & 47 & 46 & 45 & 44 & 43 & 42 & 41 & 40 & 39 & 38 & 37 & 36 & 35 & 34 & 33 & 32 & 31 & 30 & 29 & 28 & 27 & 26 & 25 & 24 & 23 & 22 & 21 & 20 & 19 & 18 & 17 & 16 & 15\end{array}$ 
NOE Experiment for compound $\mathbf{2 1}$

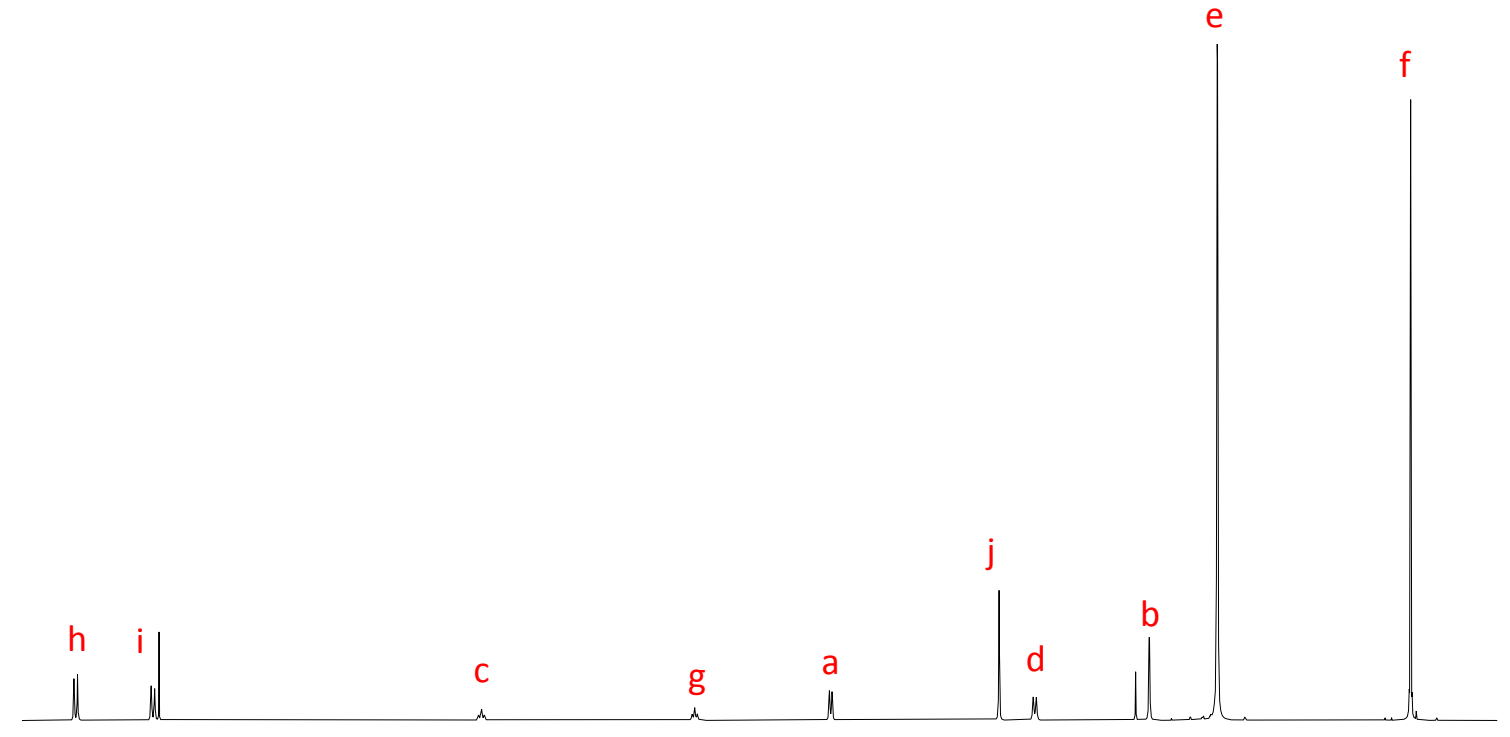

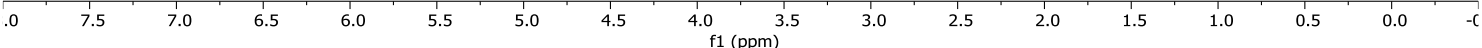
${ }^{1} H$ NMR of product 21.

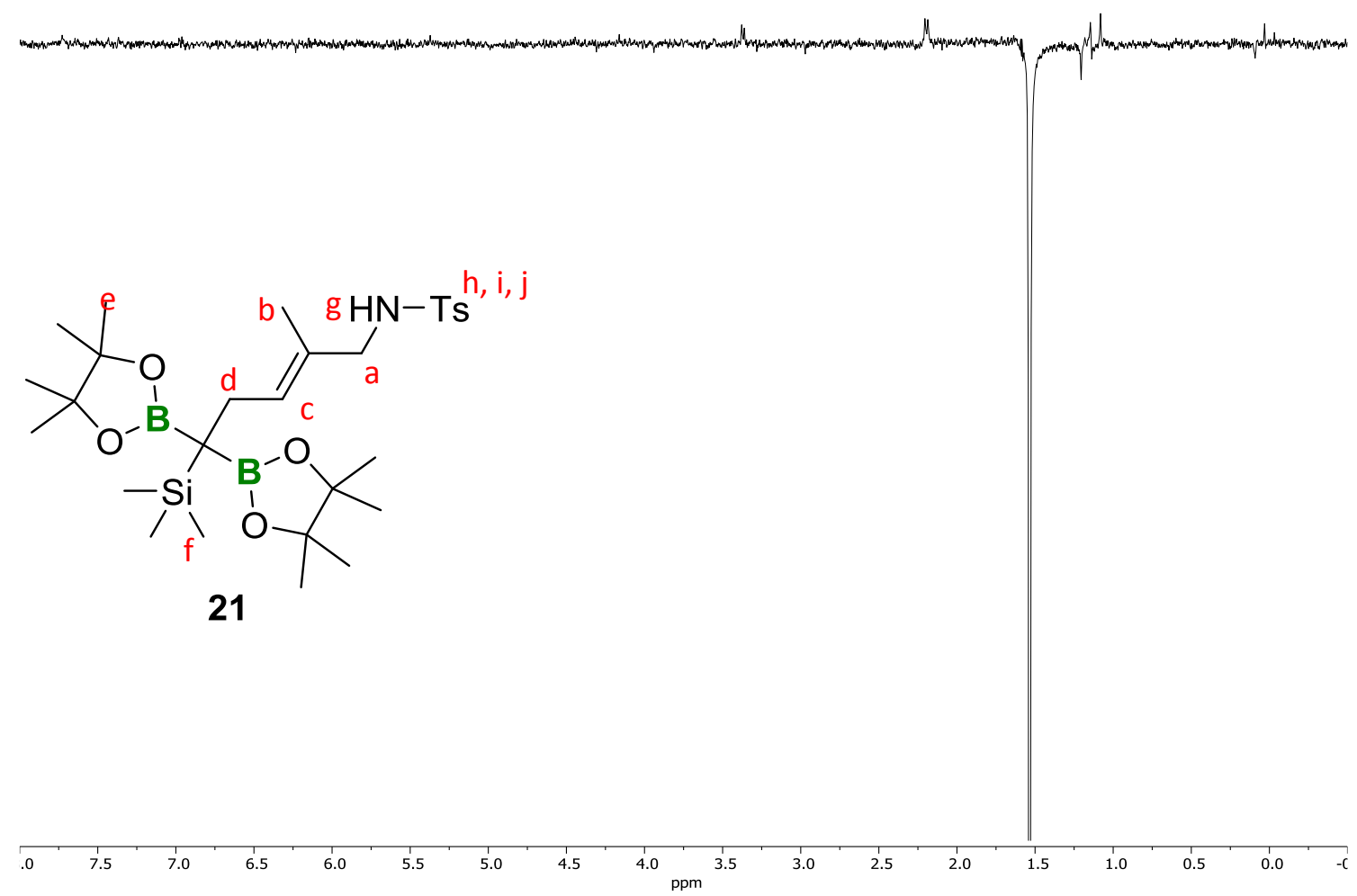

NOE of product 21 irradiating at 1.54 ppm. 


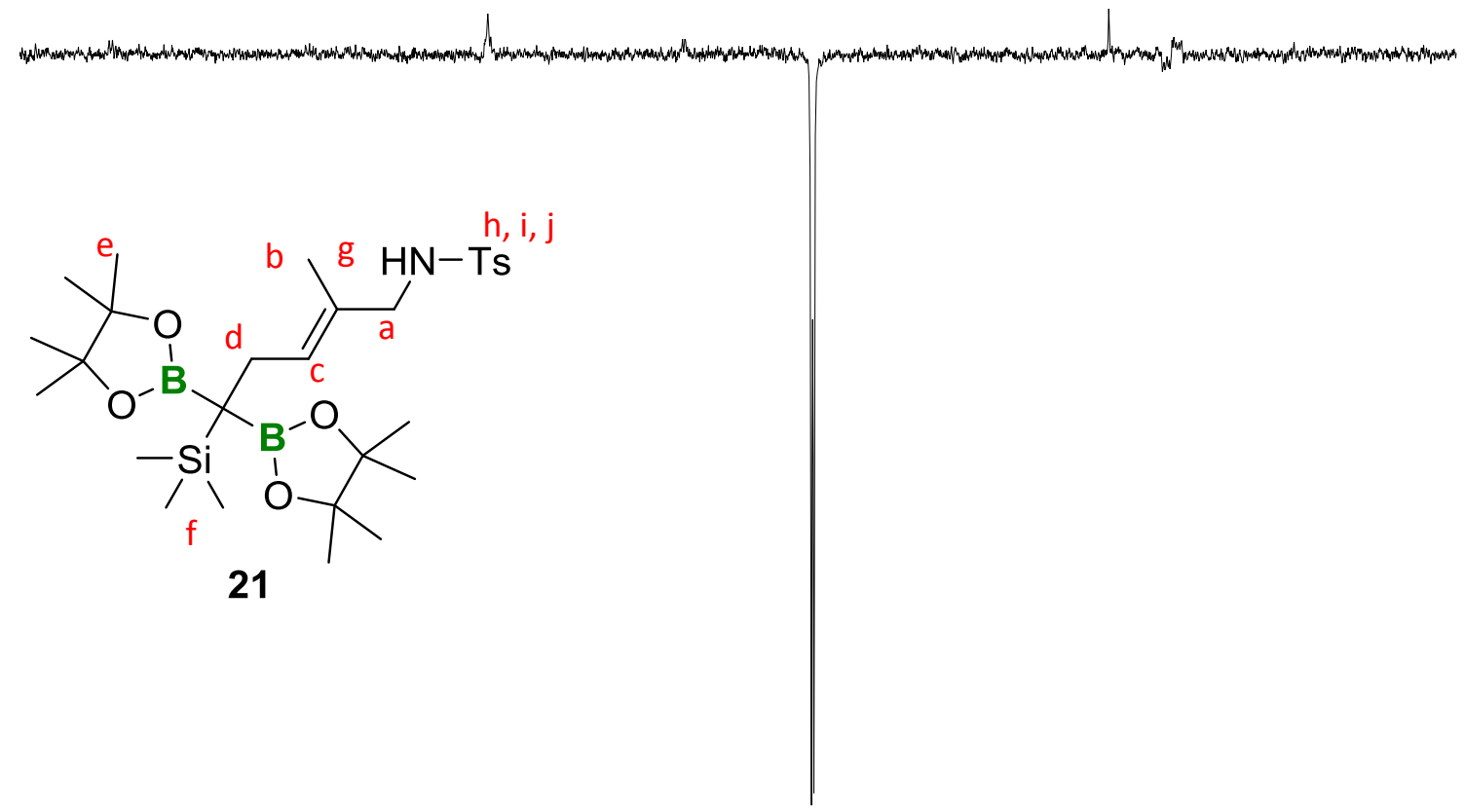

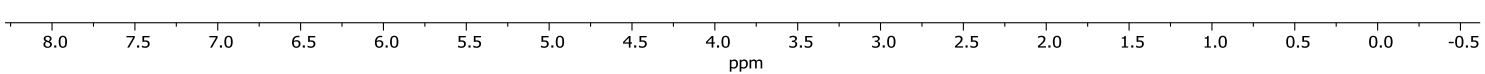

NOE of product 21 irradiating at $3.36 \mathrm{ppm}$

When we irradiated the methyl group at $1.54 \mathrm{ppm}$ (protons $\mathrm{b}$ ), the signals of the protons $\mathrm{d}$ were observed. When the protons a were irradiated at $3.36 \mathrm{ppm}$, the olefinic signal was observed, so the structure can be confirmed as the $E$ isomer of product $\mathbf{2 1}$.

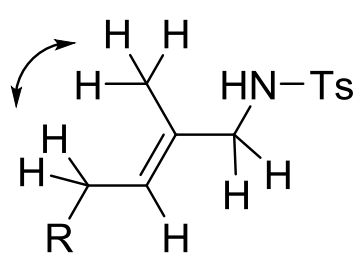

$21(E)$ 


\section{References}

[1] Knight, J. G.; Muldowney, M. P. Synlett 1995, 949.

[2] Li, H.; Shangguan, X., Zhang, Z.; Huang, S.; Zhang, Y. J.; Wang, J. Org. Lett., 2014, 16, 448.

[3] He, Z.; Zhu, Q.; Hu, X.; Wang, L.; Xia, C.; Liu, C. Org. Chem. Front. 2019, 6, 900

[4] Lacascia, E., Cuenca, A. B.; Fernández, E. Chem. Eur. J., 2016, 22, 18737. 